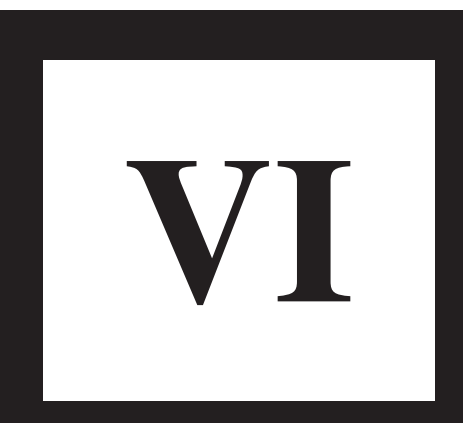

Publication VI

F. Boxberg and J. Tulkki, Theory of the electronic structure and carrier dynamics of strain-induced (Ga,In)As quantum dots, Reports on Progress in Physics, 70, pp. 1425-1471(2007).

(C) 2007 Institute of Physics Publishing

Reprinted with permission.

http://www.iop.org/journals/rop 


\title{
Theory of the electronic structure and carrier dynamics of strain-induced (Ga, In)As quantum dots
}

\author{
Fredrik Boxberg and Jukka Tulkki \\ Laboratory of Computational Engineering, Helsinki University of Technology, \\ FIN-02015 HUT, Finland \\ E-mail: fredrik.boxberg@tkk.fi \\ Received 11 September 2006, in final form 6 June 2007 \\ Published 17 July 2007 \\ Online at stacks.iop.org/RoPP/70/1425
}

\begin{abstract}
Strain-induced quantum dots (SIQD) confine electrons and holes to a lateral potential minimum within a near-surface quantum well (QW). The potential minimum is located in the QW below a nanometre-sized stressor crystal grown on top of the QW. SIQD exhibit well-resolved and prominently atomic-like optical spectra, making them ideal for experimental and theoretical studies of mesoscopic phenomena in semiconductor nanocrystals.

In this report we review the theory of strain-induced confinement, electronic structure, photonics and carrier relaxation dynamics in SIQD. The theoretical results are compared with available experimental data. Electronic structure calculations are mainly performed using the multiband envelope function approach. Many-body effects are discussed using a direct diagonalization method, albeit, for the sake of computational feasibility, within a two-band model.

The QD carrier dynamics are discussed in terms of a master equation model, which accounts for the details of the electronic structure as well as the leading photon, phonon and Coulomb interaction processes. We also discuss the quantum confined Stark effect, the Zeeman splitting and the formation of Landau levels in external fields. Finally, we review a recent theory of the cooling of radiative QD excitons by $\mathrm{THz}$ radiation. In particular we discuss the resonance charge transfer of holes between piezoelectric trap states and the deformation potential minima. The agreement between the theory and experiment is fair throughout, but calls for further investigations.
\end{abstract}

(Some figures in this article are in colour only in the electronic version)

This article was invited by Professor K Ploog

0034-4885/07/081425+47\$90.00 C 2007 IOP Publishing Ltd Printed in the UK 


\section{Contents}

List of abbreviations $\quad 1427$

1. Introduction 1428

1.1. The fabrication of SIQD 1429

1.2. The strain and the confinement of carriers 1430

1.3. Basic electronic and optical characteristics 1430

1.4. Basic experimental results 1432

2. Elastic strain $\quad 1433$

2.1. Continuum elasticity 1433

2.1.1. Piezoelectric coupling in III-V semiconductors 1434

2.2. Atomistic elasticity 1435

$\begin{array}{ll}\text { 2.2.1. Keating valence force field potential } & 1435\end{array}$

2.3. Atomistic versus continuum elasticity 1435

2.4. The elastic strain and piezoelectric potential of SIQD 1436

3. Electronic structure I-single-particle description 1437

3.1. Conventional multiband envelope wave function theory 1438

3.2. The envelope wave function theory of Burt and Foreman 1440

3.3. Effective confinement potential of electrons and holes 1442

3.4. Confined and quasi-confined electron and hole wave functions 1443

3.5. The density of electron and hole states 1444

3.6. The influence of an external electric field 1445

3.7. The influence of an external magnetic field 1448

4. Electronic structure II-many-particle description 1450

4.1. Hartree-Fock approximation 1452

4.2. Configuration interaction scheme 1453

4.3. Correlation energy 1453

4.4. Electron-hole spatial correlation 1454

5. Optical properties 1456

5.1. Photoluminescence 1457

5.2. Polarization 1459

6. Carrier dynamics $\quad 1460$

6.1. Phonon relaxation and Auger processes in SIQD 1460

6.2. Dynamical model describing carrier modulation by $\mathrm{THz}$ radiation 1461

$\begin{array}{ll}\text { 6.2.1. Underlying assumptions } & 1462\end{array}$

6.2.2. General remarks on the model parametrization 1463

6.2.3. Luminescence and carrier dynamics during continuous pumping 1464

6.2.4. Influence of THz radiation on steady-state QD luminescence 1464

6.2.5. Transient carrier dynamics under THz radiation 1464

6.2.6. THz radiation-induced delayed ground-state PL 1465

7. Summary 1465

$\begin{array}{ll}\text { Acknowledgments } & 1467\end{array}$

$\begin{array}{ll}\text { References } & 1467\end{array}$ 


\section{List of abbreviations}

2D

2DEG

3D

AE

BCC

BF

C

CB

CE

CI

CSF

CMOS

CG

CTE

CW

DFT

DOS

DP

EB

EFA

EMA

EPM

FCC

FCI

FEL

FEM

HF

HH

IC

JDOS

LA

LED

LH

LHS

LO

MBE

MC

MOSFET

MOVPE

NIR

PARPACK

PEP two dimensional

two dimensional electron gas

three dimensional

atomistic elasticity

body centered cubic

Burt-Foreman

conduction

conduction band

continuum elasticity

configuration interaction

configuration state function

complementary metal-oxide-semiconductor

conjugate gradient

coefficient of thermal expansion

continuous wave

density functional theory

density of states

deformation potential

electron beam

envelope wave function approximation

effective-mass approximation

empirical pseudopotential method

face centered cubic

full configuration interaction

free electron laser

finite element method

Hartree-Fock

heavy hole

integrated circuit

joint density of states

longitudinal acoustic

light-emitting device

light hole

left-hand side

longitudinal optical

molecular beam epitaxy

Monte-Carlo

metal-oxide-semiconductor field effect transistor

metal organic vapor phase epitaxy

near-infrared

parallel Arnoldi package

piezoelectric potential 


$\begin{array}{ll}\text { PL } & \text { photoluminescence } \\ \text { QCSE } & \text { quantum confined Stark effect } \\ \text { QD } & \text { quantum dot } \\ \text { QPC } & \text { quantum point contact } \\ \text { QW } & \text { quantum well } \\ \text { QWR } & \text { quantum wire } \\ \text { RHS } & \text { right-hand side } \\ \text { SDCI } & \text { singly and doubly excited determinants } \\ & \text { configuration interaction } \\ \text { SDTQCI } & \text { singly-, doubly-, triply- and quadruply-excited determinants } \\ & \text { configuration interaction } \\ \text { SET } & \text { single electron transistor } \\ \text { SIA } & \text { structure inversion asymmetry } \\ \text { SIQD } & \text { strain-induced quantum dot } \\ \text { SLCBB } & \text { strained linear combination of bulk bands } \\ \text { SO } & \text { split-off } \\ \text { SOI } & \text { silicon on insulator } \\ \text { SP } & \text { single particle } \\ \text { SpO } & \text { spin-orbital } \\ \text { TEM } & \text { transmission electron micrograph } \\ \text { THz } & \text { terahertz } \\ \text { VB } & \text { valence band } \\ \text { VFF } & \text { valence force field } \\ \end{array}$

\section{Introduction}

A quantum dot is a man-made solid-state structure which is able to confine one or several electrons and (or) holes to a nanometre-scale potential minimum [1-3]. The size of a typical QD ranges from a few lattice constants to a few micrometres. In this review we discuss the theory and modelling of the electronic structure and carrier dynamics of SIQD [4]. The 3D confinement of carriers in SIQD results from the combined effect of the band-edge discontinuity of a near-surface QW (vertical confinement) and strain-induced lateral confinement, caused by a nanometre-sized stressor island on top of the QW (see figure 1). The lattice mismatch between the superficial InP stressor island and the GaAs substrate induces a smooth and nearly parabolic strain deformation potential into the QW. As a result of the parabolic deformation potential, SIQD exhibit a uniquely regular and atomic-like photoluminescence spectrum. The weak potential barriers (shoulders) in the schematic band diagram of the SIQD result from the straininduced band-edge deformation. However, in a full 3D analysis including piezoelectricity (discussed in section 3) these shoulders become superimposed with piezoelectric effects and partly disappear. We will mainly discuss a particular type of SIQD, shown schematically in figure 1, where the stressor is made of InP and the QW is composed of a GaAs and InGaAs heterostructure. Our discussion, however, also gives a good qualitative reference to other SIQD structures, composed of different QW and stressor materials.

We want to emphasize the distinction between SIQD and the more extensively studied overgrown InAs QD, also depicted in figure 1. The confinement of carriers to overgrown InAs QD is primarily based on the GaAs/InAs band-edge discontinuity at the surface of the pyramid and the strain has only a secondary role. Current research into QD LED and lasers is primarily 


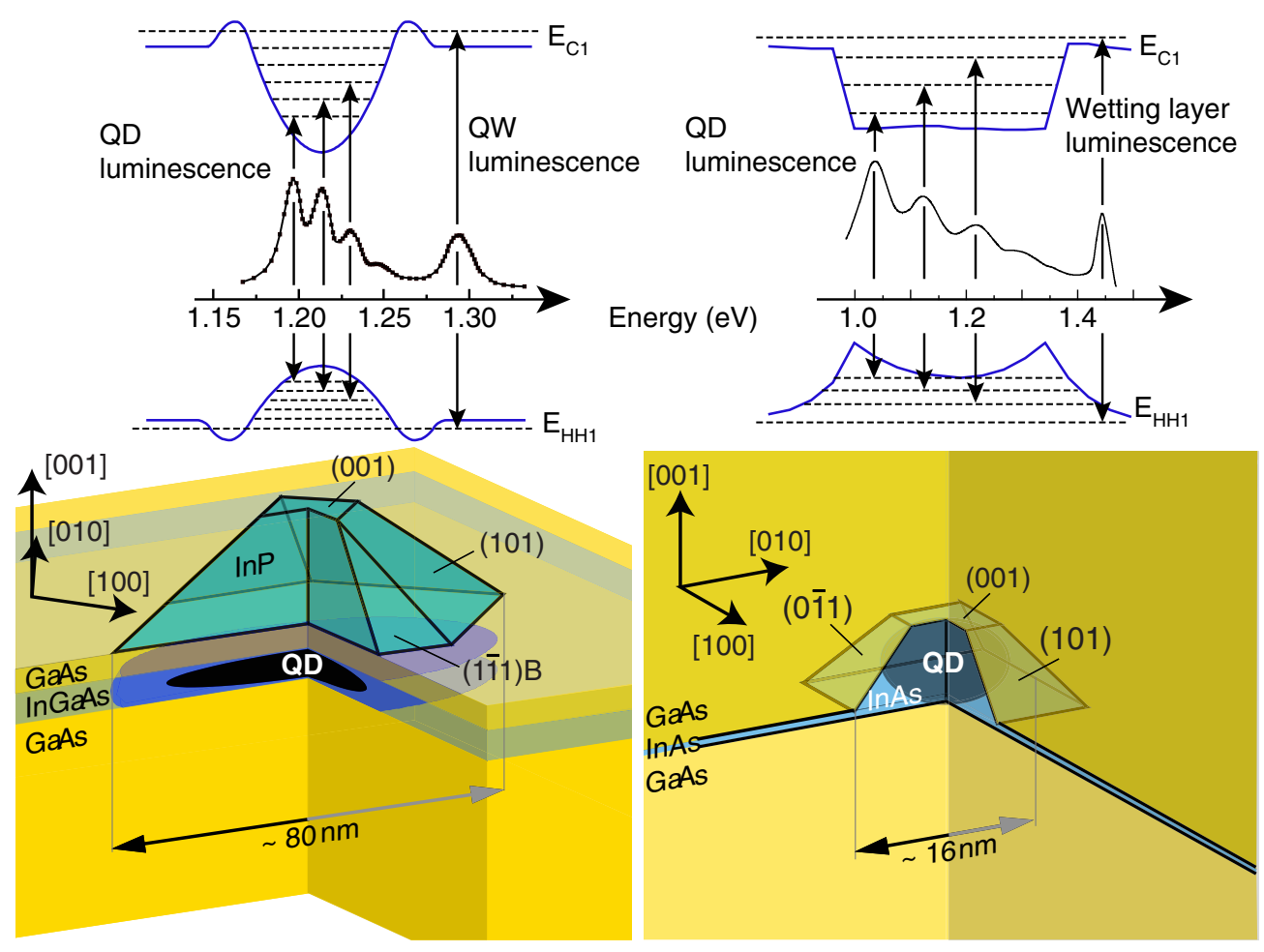

Figure 1. A schematic illustration of two different kinds of quantum dots. Lower panels show the geometry and the materials of a strain-induced QD (left) and an overgrown InAs QD (right). The upper panels show the respective real-space energy band diagrams and experimental PL data, obtained from [4] (left) and [5] (right).

based on the use of overgrown and stacked InAs quantum pyramids. An extensive literature is available regarding experimental and theoretical work on overgrown InAs quantum pyramids [1, 6-8]. Note that SIQD cannot be overgrown without a loss of carrier confinement. They are, therefore, less attractive for device applications. However, the electronic structure of SIQD is more atomic-like and the well-resolved spectral lines permit a rich variety of spectroscopic measurements. This makes SIQD ideal for the theoretical and experimental study of the basic physics of QD.

To understand the possibilities and limitations of theoretical research into SIQD, it is important also to be aware of how these nanostructures are fabricated and to have a general phenomenological view of their basic physical properties. Therefore, in the introduction we will briefly discuss the basics of the fabrication, the origin of strain, the confinement effects, the basic physical properties and the most important experimental observations. In the later chapters of our review we will go into a detailed theoretical discussion of each of these topics.

\subsection{The fabrication of SIQD}

InP stressor islands are grown on a GaAs substrate by self-organized molecular beam or vapour phase epitaxy. The islands are a result of the lattice mismatch between InP (lattice constant $\left.a_{\mathrm{InP}}=5.87 \AA\right)$ and the GaAs substrate $\left(a_{\mathrm{GaAs}}=5.65 \AA\right)$. The topmost deposited InP minimizes its strain energy during the growth process by forming coherent islands, i.e. ones 

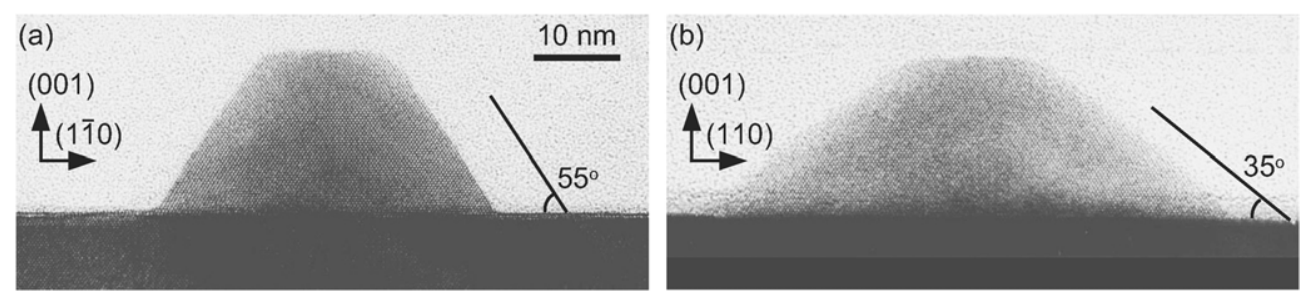

Figure 2. TEM images of an InP island that is similar to the one shown in figure 1, grown on a (0 0 1) GaAs substrate (reprinted with permission from [11]).

which are fully elastically strained and defect-free. The formation of strained islands during epitaxial growth is known as Stranski-Krastanow or self-organized growth $[9,10]$. Figure 2 shows cross-sectional TEM of a typical InP island. The shape of the island is defined by the energy minimization during the self-organized growth. This favours the formation of loworder crystal planes $\left(\left\{\begin{array}{lll}0 & 0 & 1\end{array}\right\},\left\{\begin{array}{lll}1 & 0 & 1\end{array}\right\}\right.$ and $\left.\left\{\begin{array}{lll}1 & 1 & 1\end{array}\right\}\right)$ [1 11$]$. The height and lateral width of the islands varies between 15-25 $\mathrm{nm}$ and 60-120 $\mathrm{nm}$, respectively, depending on the exact growth conditions. Typical island densities are around $10^{9} \mathrm{~cm}^{-2}[12]$.

\subsection{The strain and the confinement of carriers}

The carrier confinement of SIQD is predominantly due to hydrostatic tensile strain, induced by an InP stressor island (shown in figure 2). The tensile strain reduces the band gap and thereby creates a potential minimum (shown by a black ellipsoid in figure 1) within the QW [13]. The vertical confinement is enhanced by the GaAs barrier, mantling the QW. The result is a widely tunable QD. The depth and size of the QD carrier confinement can be tuned by changing the widths and material composition of the well and barrier layers [13] and by tuning the size of the InP island (which depends on the growth conditions) [12,14].

The type of the substrate is the most important parameter that predefines the electronic confinement and optical characteristics of the SIQD. Strain-induced quantum dots have so far been fabricated on, e.g. GaAs [4, 15-17], InP [18-20] and Si [21] substrates. The models and results for InP/GaAs/InGaAs SIQD, reviewed in this work, can also guide the analysis and understanding of other types of SIQD.

\subsection{Basic electronic and optical characteristics}

A phenomenological view of the electronic and optical properties of SIQD can be obtained using a simple two-band effective-mass model. A more realistic description, based on fully 3D multiband EFA and many-body simulations, will be given later in this review. The total $\mathrm{QD}$ confinement of electrons and holes is the sum of the $\mathrm{QW}$ carrier confinement $V_{\mathrm{QW}}^{(\mathrm{e} / \mathrm{h})}$ (vertical confinement) and the strain-induced carrier confinement $V_{\text {strain }}^{(\mathrm{e} / \mathrm{h})}$ (lateral confinement). Figure 3 shows schematically how the strain-induced confinement potential is created in the conduction band $(a)$ and valence band $(b)$ of the QW. The compressive strain, caused by the lattice mismatch between the substrate and the InGaAs QW gives rise to a uniform raising of the QW conduction and heavy-hole band-edges. The strain also gives rise to a significant splitting of the heavy and light hole bands. It is concluded that the strain greatly prefers the confinement of heavy holes to the QW. The confinement of light holes is much weaker. Since InP has a much larger lattice constant than the GaAs substrate, the coherent joint of an InP 


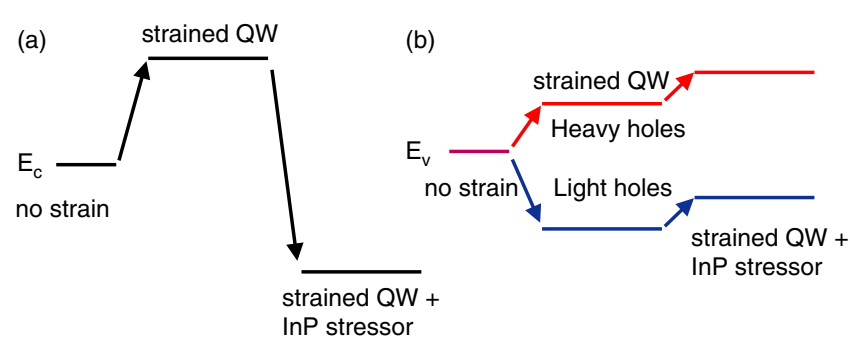

Figure 3. Schematic illustration of the strain-induced band-edge deformation for $(a)$ the conduction and $(b)$ the valence bands, evaluated in the center of the QW plane below a stressor island and, for simplicity, omitting the piezoelectric potential.

island on the GaAs top barrier will give rise to a non-biaxial local tensile strain (or relief of the compressive QW strain) in the underlying material. This will then lower (raise) the conduction (heavy hole) band-edge and give rise to a local lateral carrier confinement potential in the QW, where the band gap is roughly $110 \mathrm{meV}$ less than in the rest of the QW.

In the lowest-order axisymmetric approximation [13], the electron and hole wave functions are given by $\psi_{n, m}^{(\mathrm{e} / \mathrm{h})} \chi_{m_{\mathrm{s}}} \mathrm{e}^{\mathrm{i} m \phi}$, where $n$ is the principal quantum number governing the radial modes, $m$ (approximately a good quantum number) specifies the $z$ component of the angular momentum $\left(L_{z}=m \hbar\right)$ and $m_{\mathrm{s}}$ is the spin quantum number. The axisymmetric Schrödinger equation (neglecting intra-band coupling) for electrons (e) and holes (h) reads

$$
\begin{aligned}
\left\{-\frac{\hbar^{2}}{2 m_{0}}\left[\frac{\partial}{\partial z}\right.\right. & \left.\frac{1}{m_{z}^{(\mathrm{e} / \mathrm{h})}} \frac{\partial}{\partial z}+\frac{\partial}{r \partial r}\left(\frac{r}{m_{r}^{(\mathrm{e} / \mathrm{h})}} \frac{\partial}{\partial r}\right)-\frac{1}{m_{r}^{(\mathrm{e} / \mathrm{h})}} \frac{m^{2}}{r^{2}}\right] \\
+ & \left.V_{\mathrm{QW}}^{(\mathrm{e} / \mathrm{h})}(z)+V_{\text {strain }}^{(\mathrm{e} / \mathrm{h})}(r, z)\right\} \psi_{n, m}^{(\mathrm{e} / \mathrm{h})}(r, z)=E_{n, m}^{(\mathrm{e} / \mathrm{h})} \psi_{n, m}^{(\mathrm{e} / \mathrm{h})}(r, z),
\end{aligned}
$$

where $m_{z}=m_{z}(z)$ and $m_{r}=m_{r}(z)$ are the effective masses along and perpendicular to the axis of symmetry. The eigenstates are commonly labelled $n \Sigma, n \Pi^{ \pm}, n \Delta^{ \pm}$for $m=0, \pm 1, \pm 2, \ldots, \pm n$, where states with an equal value of $|m|$ are degenerate. The groundstate is $1 \Sigma$ and the first excited state is $1 \Pi^{ \pm}$. The states $2 \Sigma$ and $1 \Delta^{ \pm}$are very close in energy, forming the second excited level. This together with the $m= \pm l$ and spin degeneracies gives in the zero-field total level degeneracies of $2,4,6,8$, etc for the electron (hole) ground and excited states, respectively. This DOS is also reflected in the intensity amplitudes of the PL peaks (see figure 4(a)) [22]. The electron (hole) levels are, in addition, evenly spaced as a result of the parabolic confinement potential. The energy separations of the electron and hole states are typically $\Delta E^{\mathrm{e}} \approx 11-16 \mathrm{meV}$ and $\Delta E^{\mathrm{h}} \approx 1-3 \mathrm{meV}$, respectively.

If the spectral broadening is neglected, the total intensity distribution of electric dipoleallowed radiative transitions is given by the sum of several $\delta$-peaks

$$
\operatorname{PL}(E) \propto \sum_{n, m, n^{\prime}, m^{\prime}} \delta\left[E-\left(E_{n, m}^{\mathrm{e}}-E_{n^{\prime}, m^{\prime}}^{\mathrm{h}}\right)\right] \delta_{n, n^{\prime}} \delta_{m, m^{\prime}},
$$

where $m=m^{\prime}$ in the electric dipole approximation. The diagonal transitions $n=n^{\prime}$ dominate the spectrum if the fundamental angular frequencies $\omega$ of the conduction and valence band lateral harmonic potentials are equal. However, these particular selection rules are only approximative when one accounts for the inter-band coupling effects and the breaking of the axial symmetry. A surprisingly even PL peak spacing is, nevertheless, seen in the experiments (see figure 4(a)) [4]. 

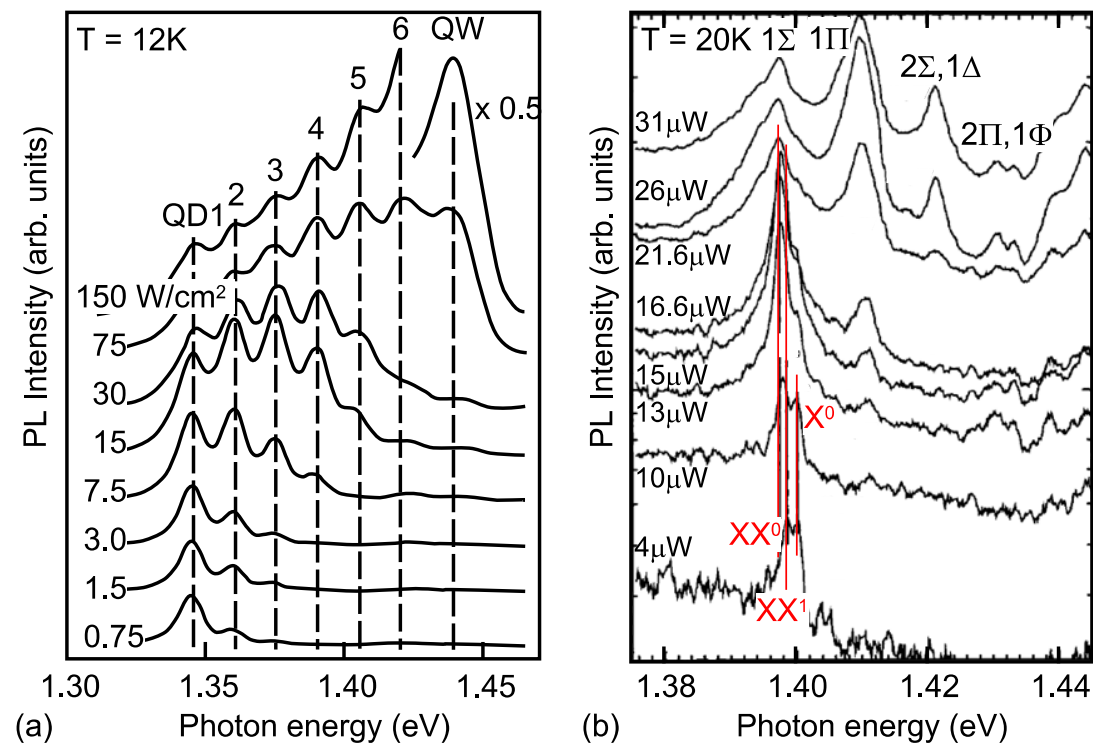

Figure 4. (a) Experimental photoluminescence of a large QD ensemble for different optical pumping intensities (reprinted with permission from [26]). The smooth peaks of the PL are due to the discrete QD levels. (b) Microluminescence spectra of a single-QD sample, for different photo-excitation intensities (reprinted with permission from [24]).

\subsection{Basic experimental results}

Figure 4 shows the experimentally measured PL of $(a)$ a large QD ensemble and $(b)$ a single isolated QD. The PL, measured as a function of optical pumping intensity, shows that the charge carriers relax towards the QD ground-state before recombining, but also how the Pauli blocking limits the number of carriers occupying individual levels simultaneously (figure $4(a)$ ). The distinct peaks of the QD PL are broadened by both homogeneous and inhomogeneous linewidth broadening [23]. The former results from the finite lifetime of the carrier eigenstates and the latter from the averaging over QD of different sizes. The details of individual radiative recombination processes should preferably be studied by single-QD spectroscopy, which lacks the inhomogeneous broadening. However, even a single-QD PL exhibits significant broadening above $20 \mu \mathrm{W}$ pumping intensities: see figure $4(b)$ [24]. This additional homogenous broadening has tentatively been attributed to charge fluctuations, but the exact mechanism is still unknown [24,25].

Figure 5 shows the prominent atomic-like features in the QD PL under homogeneous magnetic fields of different strengths [24]. This external magnetic field, parallel to the $z$-axis (the growth axis), removes the $m= \pm l$ and spin degeneracies. The magnetic dispersion of the luminescence lines is very sensitive to the symmetry of the non-degenerate single-particle electron and hole states. Both the diamagnetic shift (feature A) and the Zeeman splitting (feature B) are clearly visible in figure 5 .

The relaxation and recombination dynamics of QD have been studied using time-resolved optical spectroscopy (see figures $5(b)$ and $(c)$ ) $[27,28]$. The time evolution of the PL line intensities (figure $5(b)$ ) is in general agreement with the CW PL shown in figure 4(a). The experimental data of figure $5(b)$ show a rapid upconversion of the PL intensity, after the absorption of a femtosecond laser pulse. This is followed by a slower decline in the intensities 

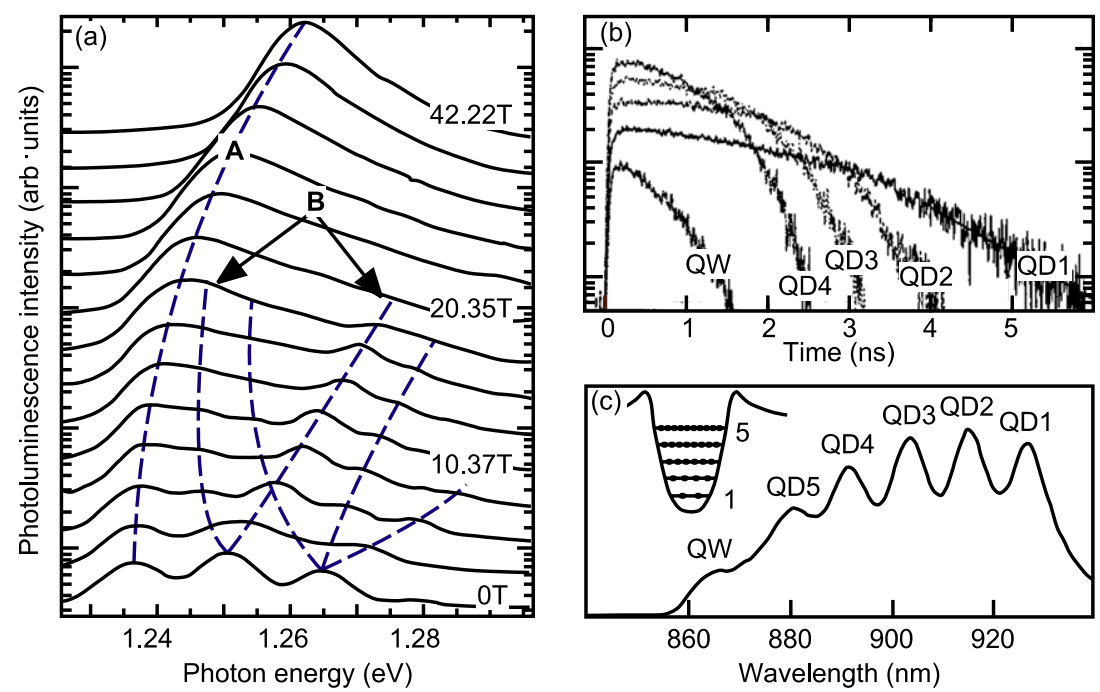

Figure 5. (a) Photoluminescence of SIQD in a magnetic field (reprinted with permission from [24]). (b) Time-resolved PL spectra of the four lowest QD peaks and of the $\operatorname{In}_{x} \mathrm{Ga}_{1-x} \mathrm{As}$ QW (reprinted with permission from [27]). (c) The CW luminescence of the same sample as in $(b)$.

of the luminescence lines. The details of the carrier relaxation from the QW to the QD and within the QD are still subject to controversy. Time-resolved spectroscopy of intra-band relaxation sequences has proved to be much more difficult with QD than with free atoms, since, unlike with free atoms, it is not possible to observe QD intra-band relaxation as sequences of well-resolved intermediate steps. The so-called phonon bottleneck effect, i.e. a reduced intraband phonon relaxation rate [29], has not been verified experimentally in the time-resolved spectra since it is hidden behind new fast Auger-like relaxation processes [28].

\section{Elastic strain}

The strain-induced carrier confinement of an SIQD is due to the pseudomorphic interface between the InP nanocrystal and the GaAs top barrier (see figure 1). The crystal lattices of InP and GaAs are joined without the formation of any dangling bonds or dislocations, despite the $3.8 \%$ difference in the lattice constants (see table 1). This is accomplished by an elastic deformation of both materials. The InP island is completely under compressive (but non-homogeneous) strain, whereas the GaAs top barrier and substrate are under tensile strain below the InP island.

An accurate description of the elastic strain, band-edge deformation and PEP is crucial for the successful modelling of SIQD, since both the electronic and photonic properties of SIQD are governed by the strain-induced confinement. In this chapter we analyse the strain of SIQD using a macroscopic CE model. We also compare the accuracy of CE, with an alternative AE model, based on the minimization of the inter-atomic potential energy.

\subsection{Continuum elasticity}

In continuum elasticity a semiconductor crystal is described as an indefinitely divisible material, neglecting all atomic-level information [30,31]. It follows that CE should be used with care 
Table 1. Elastic constants from reference [36] unless otherwise noted. The elastic constants $c_{11}$, $c_{12}$ and $c_{44}$ are given in units of $10^{11} \mathrm{~kg} \mathrm{~m}^{-1} \mathrm{~s}^{-2}$ and the piezoelectric constant is given in $\mathrm{C} \mathrm{m}^{-2}$.

\begin{tabular}{llllll}
\hline Parameter & $a(\AA)$ & $c_{11}$ & $c_{12}$ & $c_{44}$ & $e_{14}$ \\
\hline GaAs & 5.6534 & 1.190 & 0.538 & 0.595 & -0.160 \\
InAs & 6.0584 & 0.833 & 0.453 & 0.396 & -0.045 \\
AlAs & 5.662 & 1.202 & 0.570 & 0.589 & $-0.225[37]$ \\
InP & 5.8687 & 1.011 & 0.561 & 0.456 & -0.040 \\
\hline
\end{tabular}

for very small geometries [32-35]. The power of CE comes from the opportunity it provides to determine all model parameters by very accurate macroscopic experiments (see table 1) and also from the oppportunity to model piezoelectric coupling.

The general stress-strain relationship is given by Hooke's law:

$$
\boldsymbol{T}=\boldsymbol{C} \boldsymbol{S}^{\mathrm{el}},
$$

where $\boldsymbol{T}$ is the stress vector, $\boldsymbol{C}$ is the elasticity matrix and $\boldsymbol{S}^{\mathrm{el}}$ is the elastic strain vector given by $S_{i}^{\mathrm{el}}=\varepsilon_{i}-\varepsilon_{i}^{\text {th }},(i \in[x x, y y, z z, x y, y z, x z])$, where $\varepsilon_{j}$ is the total strain. The thermal strain is in turn given by $\varepsilon^{\text {th }}=\Delta T\left[\begin{array}{llll}\alpha_{x} \alpha_{y} \alpha_{z} & 0 & 0 & 0\end{array}\right]^{\mathrm{T}}$, where $\Delta T$ is the temperature change with respect to the reference temperature and $\alpha_{i}$ are the coefficients of thermal expansion. In our case, the temperature change $\Delta T$ was only used as a strain source to include the strain of the lattice-mismatched heterostructures. This was done by expanding or shrinking all materials by an amount equal to the relative lattice mismatch with respect to the substrate. For example, in the case of InP (lattice constant $a_{\mathrm{InP}}$ ) grown on GaAs (lattice constant $a_{\mathrm{GaAs}}$ ), we used $\alpha_{i}=\left(a_{\mathrm{InP}}-a_{\mathrm{GaAs}}\right) / a_{\mathrm{InP}}$, for all $i \in[x, y, z]$ and $\Delta T=1$. The strain of the crystal was finally calculated by minimizing the strain energy $E$, given by

$$
E=\frac{1}{2} \int_{\mathrm{V}} \boldsymbol{T}^{\mathrm{T}} \boldsymbol{S}^{\mathrm{el}} \mathrm{d} \boldsymbol{r}^{3}
$$

where the integration is carried out over the whole crystal.

2.1.1. Piezoelectric coupling in III-V semiconductors. The piezoelectric coupling in III-V compound semiconductors is due to the ionic bonding between Type A (cation) and Type B (anion) atoms. Displacement of the crystal atoms from their equilibrium position gives rise to local atomic dipoles and an electric field. This electric field gives rise to a force opposing the displacement. The electric interactions of the ionic crystal therefore couples the elastic strain with an internal electric field. The piezoelectric coupling can be included in the elasticity equation (2.1) (see, e.g. [35]). The vectors of the stress $\boldsymbol{T}$ and the electric flux $\boldsymbol{D}$ are in this case related to the strain $\boldsymbol{S}$ and electric field $\boldsymbol{E}$ vectors as [38]

$$
\left(\begin{array}{l}
\boldsymbol{T} \\
\boldsymbol{D}
\end{array}\right)=\left[\begin{array}{cc}
\boldsymbol{C} & \boldsymbol{e} \\
\boldsymbol{e}^{\mathrm{T}} & -\varepsilon
\end{array}\right]\left(\begin{array}{c}
\boldsymbol{S} \\
-\boldsymbol{E}
\end{array}\right) .
$$

The symmetry of the crystal enters (2.3) through the elasticity matrix $\boldsymbol{C}$ and the piezoelectric matrix $\boldsymbol{e}$. The dielectric properties of the materials are described by the dielectric matrix $\varepsilon=\varepsilon_{0} \varepsilon_{\mathrm{r}} 1_{3 \times 3}$.

The piezoelectric coupling is generally weak in lattice mismatched semiconductor heterostructures. The diagonal strain components $\varepsilon_{i i}$ are typically $\lesssim 4 \%$ and the effect of the piezoelectric coupling is about $1 \%$ of this value [35]. The direction of the polarization depends on the lattice orientation and the sign of the piezoelectric constant. The common III-V semiconductors have a negative piezoelectric constant, whereas the II-VI semiconductors have a positive piezoelectric constant. 


\subsection{Atomistic elasticity}

In atomistic elasticity the strain is calculated from the atomic displacement field of the crystal. The crystal configuration, minimizing the total strain energy, is calculated using a material-specific inter-atomic potential. It accounts for bond lengths and bond angles between neighbouring atoms. We have used the Keating VFF potential for AE reference calculations.

2.2.1. Keating valence force field potential. The Keating VFF potential $[39,40]$ expresses the energy of the crystal through a two-body part, which describes bond-stretching and a three-body part, which describes bond-bending. The total crystal energy $E_{\mathrm{VFF}}$ is obtained by summing all $N$ atoms and their $n_{i}$ nearest neighbours,

$E_{\mathrm{VFF}}=\sum_{i}^{N} \sum_{j}^{n n_{i}} \frac{3}{8} \alpha_{i j}^{(1)} \Delta d_{i j}^{2}+\sum_{i}^{N} \sum_{k>j}^{n n_{i}} \frac{3 \beta_{i j}}{8 d_{i j}^{0} d_{i k}^{0}}\left[\left(\boldsymbol{r}_{j}-\boldsymbol{r}_{i}\right) \cdot\left(\boldsymbol{r}_{k}-\boldsymbol{r}_{i}\right)-\cos \theta_{j i k}^{0} d_{i j}^{0} d_{i k}^{0}\right]^{2}$,

where $\Delta d_{i j}=\left[\left(r_{i}-r_{j}\right)^{2}-\left(d_{i j}^{0}\right)^{2}\right] / d_{i j}^{0}, \boldsymbol{r}_{i}$ is the coordinate of atom $i, \theta_{j i k}^{0}$ is the ideal angle of the bond angle $j-i-k$ and $d_{i j}^{0}$ is the equilibrium distance between atoms $i$ and $j$. The parameters $\alpha$ and $\beta$ of (2.4) are calculated by fitting the model elasticity to macroscopic elastic constants. This fitting is performed separately for each material or homogeneous alloy. Ternary alloys of the type $A_{x} B_{1-x} C$ can be approximated by effective binary alloys with atoms of the types $A B$ and $C$. The potential parameters of these alloys are obtained by interpolation between the parameters of the alloy materials.

The strained atomic structure is obtained by minimizing the total potential energy $E_{\mathrm{VFF}}$, e.g. by using the conjugate gradient method [41]. The local strain at each atom is then calculated from the relative difference between the deformed (simulated) atomic bonds and those of a strain-free crystal.

\subsection{Atomistic versus continuum elasticity}

The AE and CE models are both fitted to the macroscopic elasticity constants of bulk materials. However, only the atomistic model can describe inharmonic effects and capture the correct point-group symmetry of the atomic lattice $\left(C_{2 v}\right.$ for zinc-blend) [42]. The CE is, on the contrary, computationally more efficient for large models and describing the electro-elastic coupling of piezoelectric materials. Asymptotically, the differences between the two models decrease with increasing feature size.

There are no comparisons of CE and AE calculations for SIQD. However, the two models have been compared by Pryor et al for the strain of overgrown InAs QD [42]. The AE and $\mathrm{CE}$ yield very similar results inside the InAs dot itself. The difference was about $20 \mathrm{meV}$, measured in conduction band deformation potential energy. At the material interfaces, the AE and CE models predict very dissimilar strains as a result of the different numerical approaches to strain discontinuities. Both models should be judged critically whenever the feature size falls below $10 \mathrm{~nm}$, because the interface effects become dominant at this limit. This was also seen by Pryor et al for the $6.5 \mathrm{~nm}$ high InAs QD [42].

We have estimated the validity of the CE model for SIQD on the basis of [42] and a study of strained, corrugated QW [35]. SIQD have been found to be well suited to CE calculations because of their large size. SIQD are, in addition, free of interface apexes (which were found to be critical in corrugated QW and InAs QD) because of their planar geometry and they are well separated from the stressor island by the GaAs top barrier. Together, these factors give rise to a smooth and, within the QD, slowly varying strain field. 
Table 2. Model parameters $(T=0 \mathrm{~K})$.

\begin{tabular}{llllll}
\hline Item & $\begin{array}{l}\text { Material } \\
\text { composition }\end{array}$ & $\begin{array}{l}\text { Lattice } \\
\text { mismatch }^{\mathrm{a}}\end{array}$ & $\begin{array}{l}\text { Band gap } \\
\text { energy }(\mathrm{eV})\end{array}$ & $\begin{array}{l}\text { Valence band } \\
\text { offset }^{\mathrm{b}}(\mathrm{eV})\end{array}$ & $\begin{array}{l}\text { Dimensions } \\
(\mathrm{nm})\end{array}$ \\
\hline Stressor island & $\mathrm{InP}$ & 0.0367 & 1.4236 & -0.195 & $90.7 \times 56.7 \times 24$ \\
Top barrier & $\mathrm{GaAs}$ & 0.0000 & 1.5190 & -0.055 & Thickness 5.0 \\
Quantum well & $\mathrm{In}_{0.1} \mathrm{Ga}_{0.9} \mathrm{As}$ & 0.0071 & 1.3659 & 0.000 & Thickness 6.5 \\
Substrate & $\mathrm{GaAs}$ & 0.0000 & 1.5190 & -0.055 & - \\
\hline
\end{tabular}

${ }^{a}$ With respect to the lattice of the substrate.

b With respect to the valence band-edge of the quantum well material.

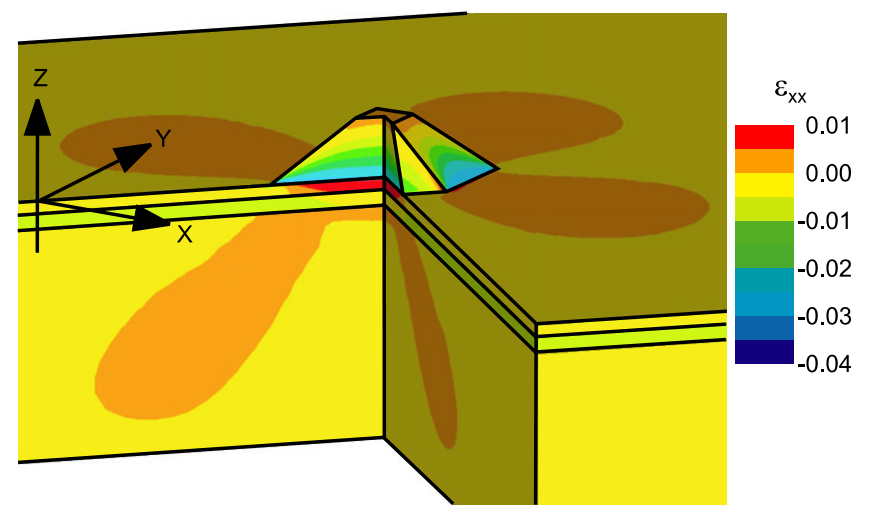

Figure 6. Strain component $\varepsilon_{x x}$ in a strain-induced quantum dot.

\subsection{The elastic strain and piezoelectric potential of SIQD}

SIQD have previously been modelled mainly with axisymmetric models $[13,43]$. The models have been surprisingly accurate, although the self-assembled stressor islands are far from axisymmetric. Detailed transmission micrographs have shown that the islands have clear and sharp facets that follow the zinc-blend lattice planes well [11]. The faceted geometry of the stressor islands gives rise to an angle-dependent confinement potential which has the same symmetry as that of the stressor island. An even greater deviation from the axisymmetric geometry is induced by the PEP $[44,45]$.

We have computed the strain of SIQD using CE and the FEM. We used both axisymmetric and fully $3 \mathrm{D}$ geometries to model the strain. The model geometry of our calculations is shown in figure 1 and the main model parameters are listed in table 2.

In the following section we will present the elastic strain in terms of cross-sectional planes, which have been found to be representative of the strain of SIQD. We emphasize, however, that the successful description of the system that was studied relies on fully 3D strain computations. Figure 6 shows the long range of the strain, outside and below the stressor island, in terms of the diagonal $\varepsilon_{x x}$ component. Figures 7 and 8 show the strain and band-edges in the QW, beneath an axial symmetric and angular stressor island. The deformations of the band-edges were calculated by (3.8) and (3.9) of section 3.4. The strain within the QW is only weakly dependent on the geometrical details of the stressor island, because of the $5 \mathrm{~nm}$ thick barrier separating the island and the QW. The PEP is, however, very sensitive to the exact shape of the stressor island. The effect of the island geometry on the electronic and photonic properties will be discussed in sections 3 and 5 . 

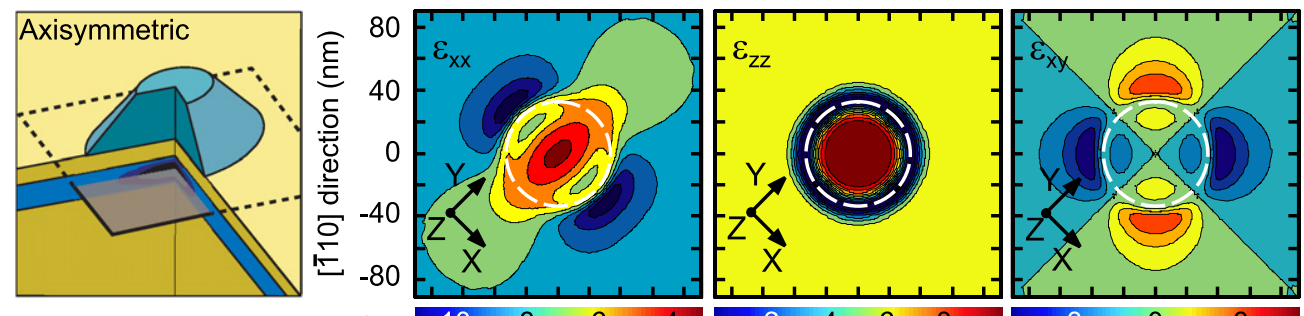

$10^{-3} x$
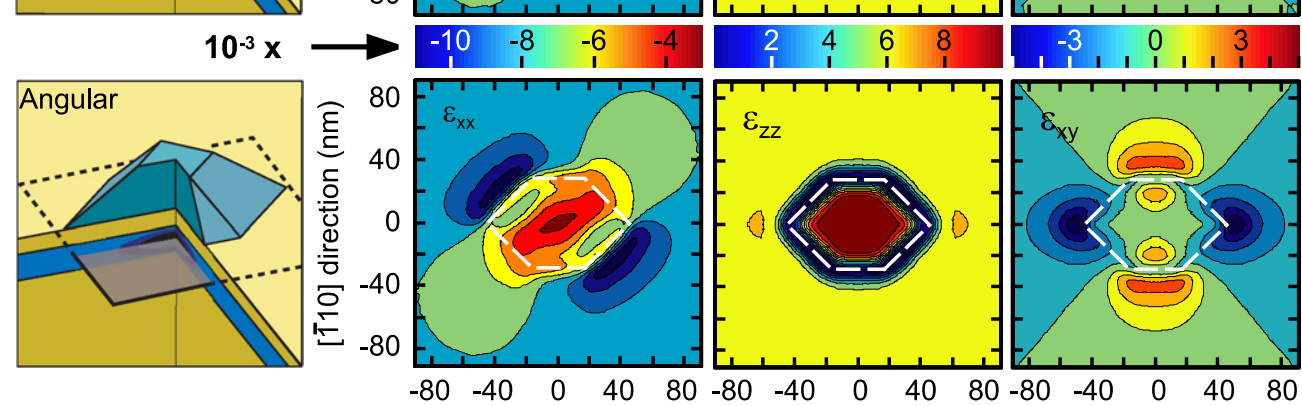

[110] direction $(\mathrm{nm})$

[110] direction $(\mathrm{nm})$

[110] direction (nm)

Figure 7. Strain in the middle of the QW, beneath an axisymmetric (upper panels) and angular (lower panels) InP stressor island. The outer contours of the islands are shown with a white dashed line. The corresponding model geometries are shown to the left.
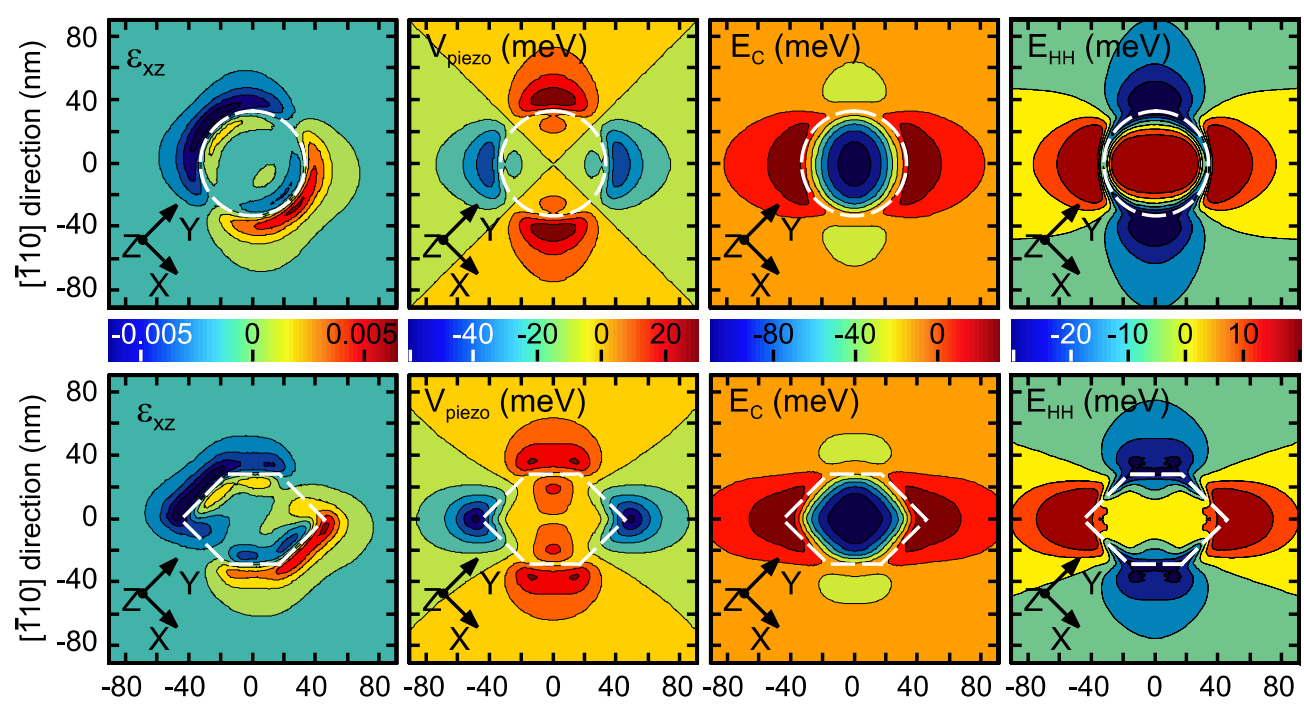

[110] direction (nm)

[110] direction $(\mathrm{nm})$

[110] direction $(\mathrm{nm})$

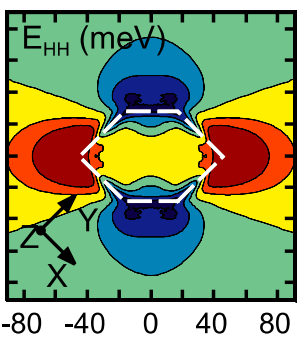

Figure 8. Shear strain $\varepsilon_{x z}$, PEP and effective potentials of electrons and holes in the middle of the $\mathrm{QW}$, beneath an axisymmetric (upper panels) and angular (lower panels) InP stressor island (outer contour shown with a white dashed line).

\section{Electronic structure I-single-particle description}

We start the discussion of the SIQD electron structure within the SP multiband EFA. The carrier-carrier correlation will be discussed separately in section 4 . The reason for the good 
predictive power of the SP EFA is not fully understood. However, there is experimental evidence that SIQD are approximately neutral (i.e. they confine equal numbers of electrons and holes) under all state filling conditions. Note the independence of PL line energies of state filling in figure 4(a). The SP approximation reproduces very well both the magnetoluminescence and the QCSE in SIQD. These observations suggest efficient screening of the carrier potentials and indicate that the correlation energies are rather small.

\subsection{Conventional multiband envelope wave function theory}

The conventional EFA theory is based on the $\boldsymbol{k} \cdot \boldsymbol{p}$ theory of the near-band-edge dispersion of bulk semiconductors. For a general description see, e.g. [46-51]. The EFA model was originally developed by Luttinger and Kohn to analyse hole states around defects in semiconductors [52]. It was later heuristically generalized to include the conduction band and applied to semiconductor heterostructures where the carrier confinement is due to the material dependence of band-edges or strain effects. The EFA model parameters always originate from a fitting to experimental or theoretical band gap and effective-mass values of the pertinent bulk semiconductors. The effects of distant bands [53,54] and elastic strain [50,51,55] are accounted for by perturbation theory (to second order in the wave vector $\boldsymbol{k}$ ).

The EFA model is best suited to semiconductors where both the conduction and the top valence bands can be described using a small number of Bloch functions $\left|u_{n k_{0}}\right\rangle$, all evaluated at a single $\boldsymbol{k}_{0}$ point. A common choice is to include eight Bloch functions in the basis set [50,51]. The EFA model is consequently accurate for calculations of confined states close to bandedges. It has, nevertheless, permitted the successful modelling of a wide variety of III-V compound semiconductor QW [45, 49, 56], QWR [35, 57, 58] and QD [8, 59-63].

Here we will review only the most common choice, where the electron eigenstates $\Psi$ are expanded in terms of the $\Gamma$ point $\left(\boldsymbol{k}_{0}=\mathbf{0}\right)$ Bloch functions, $\left|u_{v}\right\rangle$. The electron states are now given by

$$
\Psi=\sum_{v} F_{v}\left|u_{v}\right\rangle
$$

where $F_{v}$ are the envelope functions.

The $\Gamma$ point Bloch functions of the $\mathrm{C}, \mathrm{HH}, \mathrm{LH}$ and $\mathrm{SO}$ bands span the eight-dimensional subspace of the irreducible representations $\Gamma_{6}, \Gamma_{8}$ and $\Gamma_{7}$ (of the $T_{d}$ symmetry group, associated with the zinc-blend lattice). These states were used as the basis functions of our eight-band envelope functions and are given by

$$
\begin{aligned}
& u_{1 / 2,+1 / 2}^{\mathrm{c}}=\mathrm{i}|S \uparrow\rangle, \Gamma_{6} \\
& u_{1 / 2,-1 / 2}^{\mathrm{c}}=\mathrm{i}|S \downarrow\rangle, \Gamma_{6} \\
& u_{3 / 2,+3 / 2}^{\mathrm{v}}=\frac{1}{\sqrt{2}}(|X \uparrow\rangle+\mathrm{i}|Y \uparrow\rangle), \Gamma_{8} \\
& u_{3 / 2,+1 / 2}^{\mathrm{v}}=\frac{\mathrm{i}}{\sqrt{6}}(|X \downarrow\rangle+\mathrm{i}|Y \downarrow\rangle)-\mathrm{i} \sqrt{\frac{2}{3}}|Z \uparrow\rangle, \Gamma_{8} \\
& u_{3 / 2,-1 / 2}^{\mathrm{v}}=\frac{1}{\sqrt{6}}(|X \uparrow\rangle-\mathrm{i}|Y \uparrow\rangle)+\sqrt{\frac{2}{3}}|Z \downarrow\rangle, \Gamma_{8} \\
& u_{3 / 2,-3 / 2}^{\mathrm{v}}=\frac{\mathrm{i}}{\sqrt{2}}(|X \downarrow\rangle-\mathrm{i}|Y \downarrow\rangle), \Gamma_{8} \\
& u_{1 / 2,+1 / 2}^{\mathrm{v}}=\frac{1}{\sqrt{3}}(|X \downarrow\rangle+\mathrm{i}|Y \downarrow\rangle+|Z \uparrow\rangle), \Gamma_{7} \\
& u_{1 / 2,-1 / 2}^{\mathrm{v}}=-\frac{\mathrm{i}}{\sqrt{3}}(|X \uparrow\rangle-\mathrm{i}|Y \uparrow\rangle-|Z \downarrow\rangle), \Gamma_{7} .
\end{aligned}
$$


where $u_{J, J_{z}}^{\mathrm{c}}$ and $u_{J, J_{z}}^{\mathrm{v}}$ are the Bloch functions of the conduction and valence bands, $J$ is the Bloch function angular momentum and $J_{z}$ is its $z$ component.

In our model, the geometry of SIQD consists of piecewise continuous material regions, where each partial volume of a semiconductor is modelled with the pertinent bulk band parameters. The Hamiltonian consequently becomes a function of $\boldsymbol{r}$ where the matrix elements are averaged over one unit cell located at $\boldsymbol{r}$. Later, in section 3.2, we will make a comparison with the latest developments in envelope function theory. The upper half of our conventional EFA Hamiltonian $H$ is, therefore, using a generalized form, given by

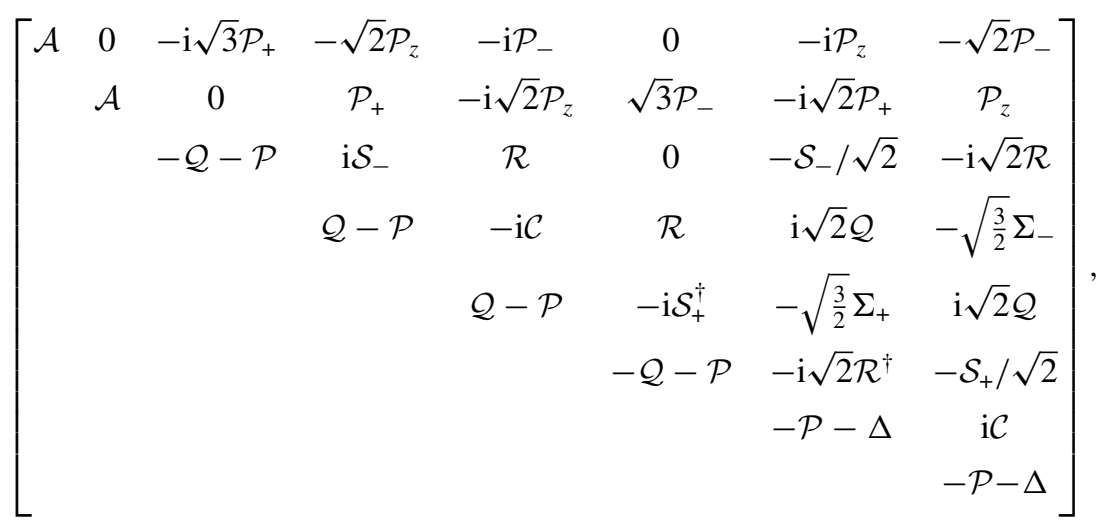

where we have defined the following symmetrized operators [49]:

$$
\begin{aligned}
& \mathcal{A}=+E_{\mathrm{c}}-\frac{\hbar^{2}}{2 m_{0}}\left(\partial_{x} \gamma_{\mathrm{c}} \partial_{x}+\partial_{y} \gamma_{\mathrm{c}} \partial_{y}+\partial_{z} \gamma_{\mathrm{c}} \partial_{z}\right)+a_{\mathrm{c}}\left(\varepsilon_{x x}+\varepsilon_{y y}+\varepsilon_{z z}\right) \\
& \mathcal{P}=-E_{\mathrm{v}}-\frac{\hbar^{2}}{2 m_{0}}\left(\partial_{x} \gamma_{1} \partial_{x}+\partial_{y} \gamma_{1} \partial_{y}+\partial_{z} \gamma_{1} \partial_{z}\right)+a_{\mathrm{v}}\left(\varepsilon_{x x}+\varepsilon_{y y}+\varepsilon_{z z}\right), \\
& \mathcal{Q}=-\frac{\hbar^{2}}{2 m_{0}}\left(\partial_{x} \gamma_{2} \partial_{x}+\partial_{y} \gamma_{2} \partial_{y}-2 \partial_{z} \gamma_{2} \partial_{z}\right)-\frac{b_{\mathrm{v}}}{2}\left(\varepsilon_{x x}+\varepsilon_{y y}-2 \varepsilon_{z z}\right), \\
& \mathcal{P}_{z}=\frac{1}{2 \sqrt{3}}\left[\left(\partial_{z} P_{0}+P_{0} \partial_{z}\right)-\left(\partial_{x} B \partial_{y}+\partial_{y} B \partial_{x}\right)\right] \\
& \mathcal{P} \pm=\frac{1}{2 \sqrt{6}}\left[\left(\partial_{x} P_{0}+P_{0} \partial_{x}\right) \pm \mathrm{i}\left(\partial_{y} P_{0}+P_{0} \partial_{y}\right)\right. \\
& \left.\left.\mathcal{S}=\mathcal{S}_{-}=S_{+}^{\dagger}=\Sigma_{-}=\Sigma_{+}^{\dagger} B \partial_{z}+\partial_{z} B \partial_{y}\right) \mp \mathrm{i}\left(\partial_{x} B \partial_{z}+\partial_{z} B \partial_{x}\right)\right] \\
& =-\sqrt{3} \frac{\hbar^{2}}{2 m_{0}}\left[\left(\partial_{x} \gamma_{3} \partial_{z}+\partial_{z} \gamma_{3} \partial_{x}\right)-\mathrm{i}\left(\partial_{y} \gamma_{3} \partial_{z}+\partial_{z} \gamma_{3} \partial_{y}\right)\right]+d_{\mathrm{v}}\left(\mathrm{i} \varepsilon_{y z}-\varepsilon_{x z}\right), \\
& \mathcal{R}=\sqrt{3} \frac{\hbar^{2}}{2 m_{0}}\left[\left(\partial_{x} \gamma_{2} \partial_{x}-\partial_{y} \gamma_{2} \partial_{y}\right)-\mathrm{i}\left(\partial_{x} \gamma_{3} \partial_{y}+\partial_{y} \gamma_{3} \partial_{x}\right)\right]+\frac{\sqrt{3} b_{\mathrm{v}}}{2}\left(\varepsilon_{x x}-\varepsilon_{y y}\right)-\mathrm{i} d_{\mathrm{v}} \varepsilon_{x y} \\
& \mathcal{C}=0 .
\end{aligned}
$$

In (3.3) and (3.4a)-(3.4h), $E_{\mathrm{c}}\left(E_{\mathrm{v}}\right)$ is the position of the conduction (valence) band-edge, $\Delta$ is the spin-orbit splitting energy, $P_{0}$ is the $\boldsymbol{k} \cdot \boldsymbol{p}$ matrix element of the conduction-valence band coupling and $B$ is the Kane's band parameter related to the inversion asymmetry of a zinc-blend crystal. The parameter $B$ has been included in $(3.4 a)-(3.4 h)$ for the sake of completeness and future reference, although in our simulations we have adopted the common choice of neglecting 
the inversion symmetry-breaking by setting $B=0[8,33,51,59,64]$. Furthermore, $a_{\mathrm{c}}, a_{\mathrm{v}}, b_{\mathrm{v}}$ and $d_{\mathrm{v}}$ are the deformation potentials related to the strain. The modified Luttinger parameters $\gamma_{i}$ are related to the original Luttinger parameters $\gamma_{i}^{\mathrm{L}}$ by

$\gamma_{1}=\gamma_{1}^{\mathrm{L}}-\frac{2 m_{0} P_{0}^{2}}{3 \hbar^{2} E_{\mathrm{g}}}, \quad \gamma_{2}=\gamma_{2}^{\mathrm{L}}-\frac{m_{0} P_{0}^{2}}{3 \hbar^{2} E_{\mathrm{g}}}, \quad \gamma_{3}=\gamma_{3}^{\mathrm{L}}-\frac{m_{0} P_{0}^{2}}{3 \hbar^{2} E_{\mathrm{g}}}$.

\subsection{The envelope wave function theory of Burt and Foreman}

The conventional EFA model described in section 3.1 is based on the assumption that the electron and hole confinement potential is a gentle (varying slowly within a single unit cell) perturbation of the pertinent bulk reference potential. The real-space equations satisfied by the envelope function are equivalent to the $\boldsymbol{k} \cdot \boldsymbol{p}$ eigenvalue problem of bulk crystals [65], except for the material parameters which are allowed to be functions of the position. When applied to semiconductor quantum structures, it is implicitly assumed that the EFA equations are also valid across an atomically abrupt material interface. The original derivation by Luttinger and Kohn [52] is, however, not strictly applicable to this case and a lot of work has been done to derive a consistent EFA theory [66-72], with position-dependent material parameters.

Burt $[73,74]$ and Foreman $[75,76]$ have developed an alternative formulation of the envelope function theory for semiconductor heterostructures, starting from the single-particle Schrödinger equation. The BF envelope function theory circumvents the issue of correct continuity conditions at material interfaces as it accounts from the beginning for possible material discontinuities in terms of material-independent basis functions [73]. In particular, since the substitution $\hat{\boldsymbol{k}} \rightarrow-\mathrm{i} \nabla$ is not made in the BF theory, the need to symmetrize the position-dependent Luttinger and Kohn EFA Hamiltonian never arises either. As pointed out by Foreman [76], the symmetrization is not needed to make the EFA Hamiltonian Hermitian. The BF derivation shows, therefore, that the operator symmetrization, in the conventional EFA Hamiltonian, is not correct [75-77]. The symmetrization gives rise to a local numerical error everywhere where the material parameters are functions of the position.

In order to write the BF Hamiltonian in terms of experimentally measurable bulk dispersion parameters, one is forced to approximate the exact form of the BF envelope function Hamiltonian (equation (6.4) of [73]) [78,77]. Doing so allows us to compare the conventional EFA model (see section 3.1) and the BF model [75] in terms of the individual elements of the eight-band Hamiltonians. The BF Hamiltonian of [75] is based on the following approximations of equation (6.4) of [73]: (i) all nonlocal and interface terms were omitted; (ii) every position-dependent function was approximated as piecewise constant; (iii) the denominator of Burt's equation (6.4) was replaced with $\left[E-H_{r r}(\boldsymbol{r})\right] \rightarrow\left[E_{\mathrm{v}}(\boldsymbol{r})-E_{r}(\boldsymbol{r})\right]$, where $E_{\mathrm{v}}(\boldsymbol{r})$ and $E_{r}(\boldsymbol{r})$ are the position-dependent valence band maximum and band-edge of the distant state $u_{r}$, respectively.

A comparison of conventional and BF eight-band Hamiltonians shows that the latter can be obtained from the former through the following replacements in $(3.4 a)-(3.4 h)$ :

$\mathcal{A}$ unchanged,

$\mathcal{P}$ unchanged,

$\mathcal{Q}$ unchanged,

$\mathcal{P}_{z}=\frac{1}{\sqrt{3}} P_{0} \partial_{z}-\frac{1}{2 \sqrt{3}}\left(\partial_{x} B \partial_{y}+\partial_{y} B \partial_{x}\right)$,

$\mathcal{P}_{ \pm}=\frac{1}{\sqrt{6}} P_{0}\left(\partial_{x} \pm \mathrm{i} \partial_{y}\right)-\frac{1}{2 \sqrt{6}}\left[\left(\partial_{y} B \partial_{z}+\partial_{z} B \partial_{y}\right) \pm \mathrm{i}\left(\partial_{x} B \partial_{z}+\partial_{z} B \partial_{x}\right)\right]$, 


$$
\begin{aligned}
& \mathcal{S}_{ \pm}=\frac{\hbar^{2}}{2 \sqrt{3} m_{0}}\left[\left(\partial_{x} \pm \mathrm{i} \partial_{y}\right)\left(1+\gamma_{1}-2 \gamma_{2}-6 \gamma_{3}\right) \partial_{z}-\partial_{z}\left(1+\gamma_{1}-2 \gamma_{2}\right)\left(\partial_{x} \pm \mathrm{i} \partial_{y}\right)\right] \\
& \quad+d_{\mathrm{v}}\left(\mathrm{i} \varepsilon_{y z}-\varepsilon_{x z}\right), \\
& \Sigma_{ \pm}=\frac{\hbar^{2}}{6 \sqrt{3} m_{0}}\left[\partial_{z}\left(1+\gamma_{1}-2 \gamma_{2}-12 \gamma_{3}\right)\left(\partial_{x} \pm \partial_{y}\right)-\left(\partial_{x} \pm \partial_{y}\right)\left(1+\gamma_{1}-2 \gamma_{2}+6 \gamma_{3}\right) \partial_{z}\right],
\end{aligned}
$$

$\mathcal{R}$ unchanged,

$\mathcal{C}=\frac{\hbar^{2}}{3 m_{0}}\left[\partial_{z}\left(1+\gamma_{1}-2 \gamma_{2}-3 \gamma_{3}\right)\left(\partial_{x}-\mathrm{i} \partial_{y}\right)-\left(\partial_{x}-\mathrm{i} \partial_{y}\right)\left(1+\gamma_{1}-2 \gamma_{2}-3 \gamma_{3}\right) \partial_{z}\right]$.

When generating the lower half of $H$ in (3.3), it is important to bear in mind that the conjugate transpose $H_{i j}=H_{j i}^{\dagger}$ reverses the operator ordering. This ensures that $H$ is Hermitian.

In [75] the $f$ symmetric distant states were omitted, because the contribution of $f$ orbitals to the valence band was assumed to be small. This approximation was already done in $[79,80]$. It is likely to influence only on first-principle calculations of the material parameters. The conventional eight-band EFA models do, however, often account for the $f$ symmetric distant states $[50,65]$.

The lack of inversion symmetry (Kane's band parameter $B$ ) of III-V semiconductors enters the bulk eight-band $\boldsymbol{k} \cdot \boldsymbol{p}$ Hamiltonian through the interaction between the conduction band $(|S\rangle)$, the valence bands $(|X\rangle,|Y\rangle,|Z\rangle)$ and the distant $\Gamma_{15}$ states. It follows that the six-band BF envelope function theory, as formulated in [75], does not contain Kane's $B$ parameter of the inversion asymmetry. There is, however, no a priori reason that would prevent the inclusion of the inversion symmetry-breaking in an eight-band BF model and we have accordingly included this term in (3.6e) for completeness and for future reference. Note that the symmetrical form of the $B$ dependent terms in (3.6a)-(3.6i) is not due to the conventional operator symmetrization but is a result of the BF Hamiltonian and the interaction between the conduction and valence bands.

A comparison of the conventional EFA model and the BF model shows that the former suffers from an inaccurate derivation of effective-mass equations from the analytically correct bulk $\boldsymbol{k} \cdot \boldsymbol{p}$ model. This is related to the unjustified symmetrization of the Hamiltonian. The band mixing is consequently overestimated, especially at the interfaces between materials with very dissimilar Luttinger parameters. This overestimation can lead to nonphysical solutions, particularly for strong magnetic fields [81].

The BF model should be considered as superior to the conventional EFA model. However, the BF model (given in (3.6a)-(3.6i)) is still an approximation [78] of the exact EFA equations of [73]. The exact EFA equations are difficult to implement numerically, because it is obviously not feasible to extract the values of all model parameters from experiments [81].

Several numerical comparisons of the conventional and the BF models have been carried out [82-84]. On the basis of figures 6-8 of [82] it seems that the discrepancies between the two models decrease with an increasing number of basis functions. The discrepancies between the band dispersions of a $10 \mathrm{~nm}$ thick lattice-matched GaAs/AlGaAs QW were small when eight-band models were used. A similar six-band comparison was performed by Boujdaria et al for a strained QW. The conclusion there was that the two methods are in relatively fair agreement at the zone centre, if the differences in Luttinger parameters between the well and barrier materials do not exceed $15 \%$. Similar observations were achieved in a comparison of a tight-binding calculation with conventional EFA and BF-type calculations of different QW [85]. Mlinar et al have modelled QD in a magnetic field using the two models [81] and concluded that only when the QD thickness is larger than $6 \mathrm{~nm}$ do the results obtained by the 
two models become identical in the magnetic field range under consideration. This was found to be the result of the different treatment of material interfaces, which becomes important for very thin geometries.

A lot of work has been done on achieving an understanding of the 'spurious states' or 'band gap states' commonly observed in the conventional EFA model $[62,69,86]$. However, BF has not provided a complete solution to this [76]. The spurious states are in fact a property of the truncated bulk $\boldsymbol{k} \cdot \boldsymbol{p}$ Hamiltonian [77].

The electronic structure calculations presented in this review were calculated using the conventional EFA model and using symmetrized Hamiltonian operators. The results and conclusions are, however, not likely to be changed by the BF model, since the Luttinger parameters of $\mathrm{GaAs}$ and $\mathrm{In}_{0.1} \mathrm{Ga}_{0.9} \mathrm{As}$ are very similar. The non-symmetrized form of the $\mathrm{BF}$ Hamiltonian is therefore likely to give fairly small material boundary corrections. In our EFA simulations of SIQD we did observe spurious states and removed them by reducing the $E_{\mathrm{p}}$ band coupling, simultaneously adjusting the conduction band parameter, which is given by [49]

$$
\gamma_{\mathrm{c}}=\frac{1}{m_{\mathrm{c}}^{*}}-\frac{E_{\mathrm{p}}\left(E_{\mathrm{g}}+2 \Delta_{\mathrm{so}} / 3\right)}{E_{\mathrm{g}}\left(E_{\mathrm{g}}+\Delta_{\mathrm{so}}\right)} .
$$

This technique ensured that the bulk conduction band effective mass of our numerical model remained in agreement with the experimental target value $m_{\mathrm{c}}^{*}$ despite the reduced conductionvalence band coupling [51].

\subsection{Effective confinement potential of electrons and holes}

The confinement of electrons and holes to SIQD is a combined result of the local strain field, caused by the InP stressor (see section 2.4 with figures 7 and 8 ) and the QW potential. In the electron structure calculations reported here, we included the full (up to first order in the wave vector $\boldsymbol{k}$ ) eight-band strain perturbation of the band structure. However, it is not feasible to visualize the complete $3 \mathrm{D}$ carrier confinement potential, since it also includes a strain and material-dependent band coupling. Figures 9 and 10 consequently show only visual estimates of the effective confinement potential at two selected cross-sections of $(a)$ electrons and $(b)$ holes, plotted with respect to the QW band-edge energy. The outer contours of the InP island are shown in figure 9 with black lines. Figures 9 and 10 are based on a bulk description of the strain-induced band-edge deformation of the conduction and $\mathrm{HH}$ band-edges as described by Bahder in [51]. These subbands are the main components of the electron and hole ground-states in the QD. The electron potential $\delta E_{\mathrm{C}}$ is in this approximation given by [51]

$$
\delta E_{\mathrm{C}}=a_{\mathrm{c}}\left(\varepsilon_{x x}+\varepsilon_{y y}+\varepsilon_{z z}\right)+q V_{\mathrm{PEP}},
$$

where $q=-|e|$ is the electron charge. The hole potential $\delta E_{\mathrm{V}}$ of figures $9(b)$ and $10(b)$ contains the main band-edge deformation of the $\mathrm{HH}$ band and the piezoelectric potential $V_{\mathrm{PEP}}$ [51]:

$$
\delta E_{\mathrm{V}}=-a_{\mathrm{v}}\left(\varepsilon_{x x}+\varepsilon_{y y}+\varepsilon_{z z}\right)+\sqrt{E_{\mathrm{s}}}+q V_{\mathrm{PEP}},
$$

where

$E_{\mathrm{s}}=\frac{b_{\mathrm{v}}^{2}}{2}\left[\left(\varepsilon_{x x}-\varepsilon_{y y}\right)^{2}+\left(\varepsilon_{x x}-\varepsilon_{z z}\right)^{2}+\left(\varepsilon_{y y}-\varepsilon_{z z}\right)^{2}\right]+d_{\mathrm{v}}^{2}\left(\varepsilon_{x y}^{2}+\varepsilon_{y z}^{2}+\varepsilon_{x z}^{2}\right)$

and $a_{\mathrm{c}}, a_{\mathrm{v}}, b_{\mathrm{v}}$ and $d_{\mathrm{v}}$ are the deformation potential constants.

The strain-induced carrier confinement weakens with increasing distance from the InP stressor island. This gives rise to a QD potential, which also depends on the $z$ coordinate. The $z$ dependence of the strain field leads to a reduction in strain-induced QD confinement with increasing top barrier thickness $[4,13]$. The QD confined states approach the pertinent lowest QW levels for a thick top barrier. 

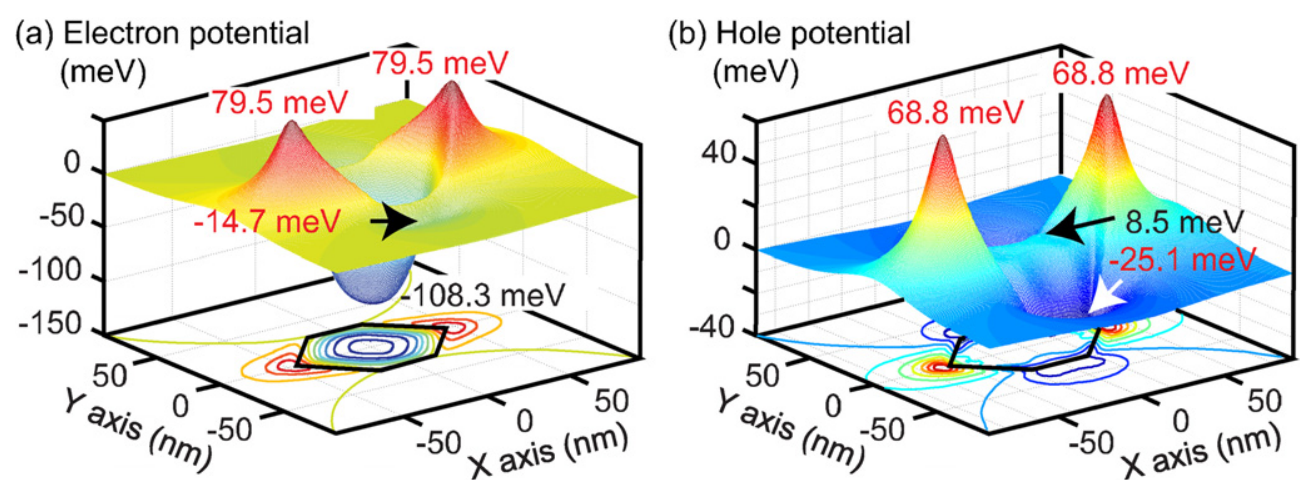

Figure 9. Potential of ( $a$ ) electrons and $(b)$ holes (based on a bulk $\Gamma$ point estimation [51]), plotted for the middle cross-section of the QW of a SIQD. The PEP side minima of electrons are only $-14.7 \mathrm{meV}$ deep whereas the PEP side minima of the holes are $68.8 \mathrm{meV}$ deep.
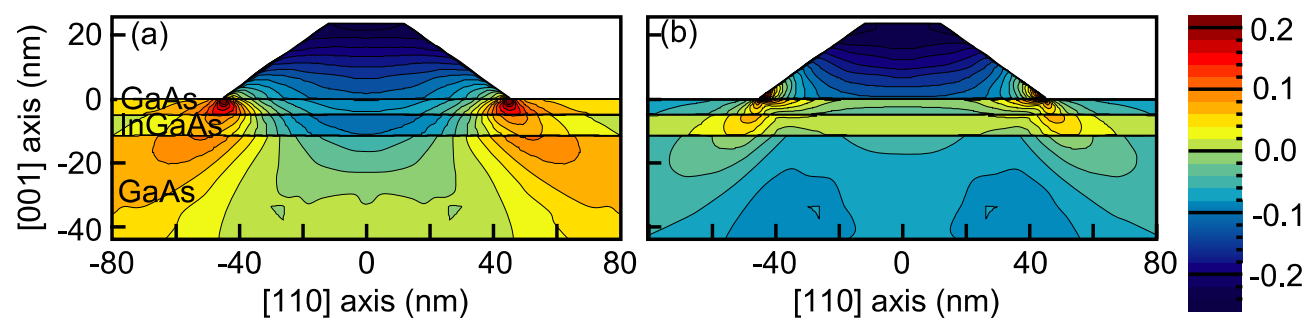

Figure 10. Potential in eV of $(a)$ electrons and $(b)$ holes at a cross-sectional [ $\left[\begin{array}{lll}0 & 0 & 1\end{array}\right]-\left[\begin{array}{lll}1 & 1 & 0\end{array}\right]$ plane, through the centre of the SIQD and the InP stressor island.

Another prominent consequence of the $z$ dependent strain field is the lack of symmetry with respect to the midplane of the $\mathrm{QW}\left(z_{0}=-8.25 \mathrm{~nm}\right.$ in figure 10). Because of the $z$ dependence of the confinement potential, the parity with respect to $z_{0}$ is no longer a good quantum number. This leads to anti-crossings of spectral lines when any two states with the same set of symmetry-related quantum numbers come close in energy, e.g. as the result of an external magnetic field $[87,88]$.

Since our calculations include the SIA, they also automatically account for the related corrections in the QD eigenenergies. We note, however, that we have not observed any zero-field spin-splitting which would result from the SIA. SIA-related spin-splitting has been reported for asymmetric QW [89]. This splitting, also approximated by the Rashba Hamiltonian [90], is, however, zero for $\boldsymbol{k}=\mathbf{0}$, suggesting that there should be no SIA-related spin-splitting in SIQD.

\subsection{Confined and quasi-confined electron and hole wave functions}

The SIQD carrier confinement potentials, shown in figures 9 and 10, contain three different carrier potential minima: (i) the strain-induced DP minimum, which is important for both electrons $(\sim-110 \mathrm{meV})$ and holes $(9 \mathrm{meV})$ and (ii-iii) two PEP minima, important only for the holes $(\sim 70 \mathrm{meV})$. The PEP does induce potential minima of electrons as well; however, these are very shallow $(\sim-10 \mathrm{meV})$ and can be neglected.

We can distinguish between three kinds of confined electron and hole states: (1) the electron states confined to the centre DP minimum (these states are denoted by $\left|e_{i}\right\rangle$ ); (2) the hole states 

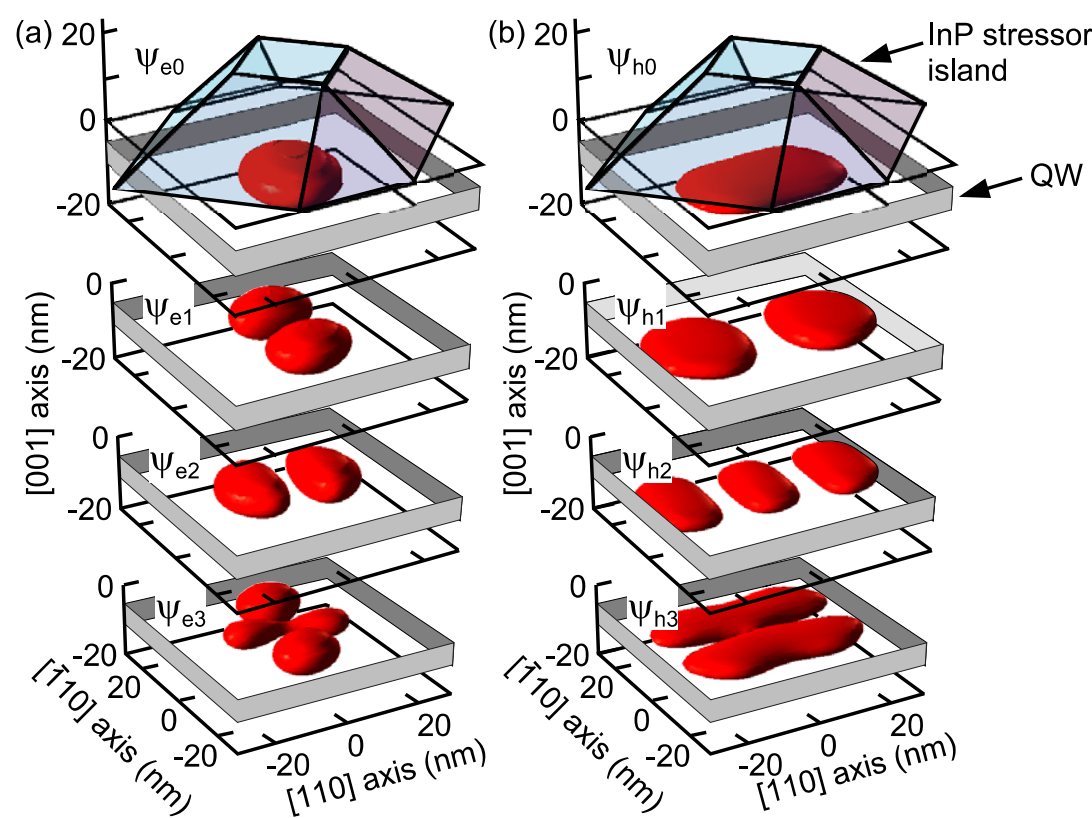

Figure 11. (a) Electron and $(b)$ hole probability densities of the four eigenstates, lowest in energy. The InP stressor island is shown in blue with respect to the ground-states $\Psi_{(\mathrm{e} / \mathrm{h}) 0}$. The position of the QW is shown in grey.

confined to the centre DP minimum (denoted by $\left|h_{i}\right\rangle$ ); and (3) the hole states confined to the PEP minima (denoted by $\left|p_{i}\right\rangle$ ). In addition to the confined states there are $2 \mathrm{D}$ electron and hole continuum states in the QW. A strict distinction between confined and delocalized states is, however, not always possible as there are indeed a large number of quasi-confined hole states with wave functions that are distributed over all three potential minima. The eigenenergies of these states overlap with the energies of the $\left|h_{i}\right\rangle$ DP states or the 2D continuum of QW hole states. These quasi-confined states are important for the optical properties of SIQD as many of them have a non-negligible recombination amplitude with the confined electron states.

There are no PEP electron states, because the PEP is of lesser magnitude along the long side of the InP stressor island, where it attracts the negative charge (marked by ' $-14.7 \mathrm{meV}$ ' in figure 9). The DP minimum of electrons is, in addition, much larger and deeper than the DP minimum of holes (cf (3.8) and (3.9)). The confinement of electrons and holes to different locations gives rise to optically dark hole states in the PEP minima, since these $\left|p_{i}\right\rangle$ states have a negligible wave function overlap with the $\left|e_{i}\right\rangle$ electron states.

Figure 11 shows the probability densities of the lowest optically active eigenstates of $(a)$ electrons and $(b)$ holes, in the DP minima. The topmost transparent surfaces represent the facets of the InP stressor island and the opaque vertical surfaces show the position of the QW. The probability density of the electron ground-state wave function $\psi_{e 0}$ is nearly axisymmetric

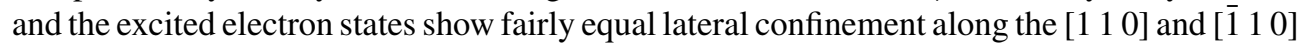
axes, whereas the hole densities of figure $11(b)$ are all elongated along the $\left[\begin{array}{lll}1 & 1 & 0\end{array}\right]$ axis as a result of the PEP.

\subsection{The density of electron and hole states}

Figure 12 shows the total density of hole and electron states in the whole SIQD. The influence of the PEP on the DOS cannot be overemphasized. The PEP minima contain many hole 


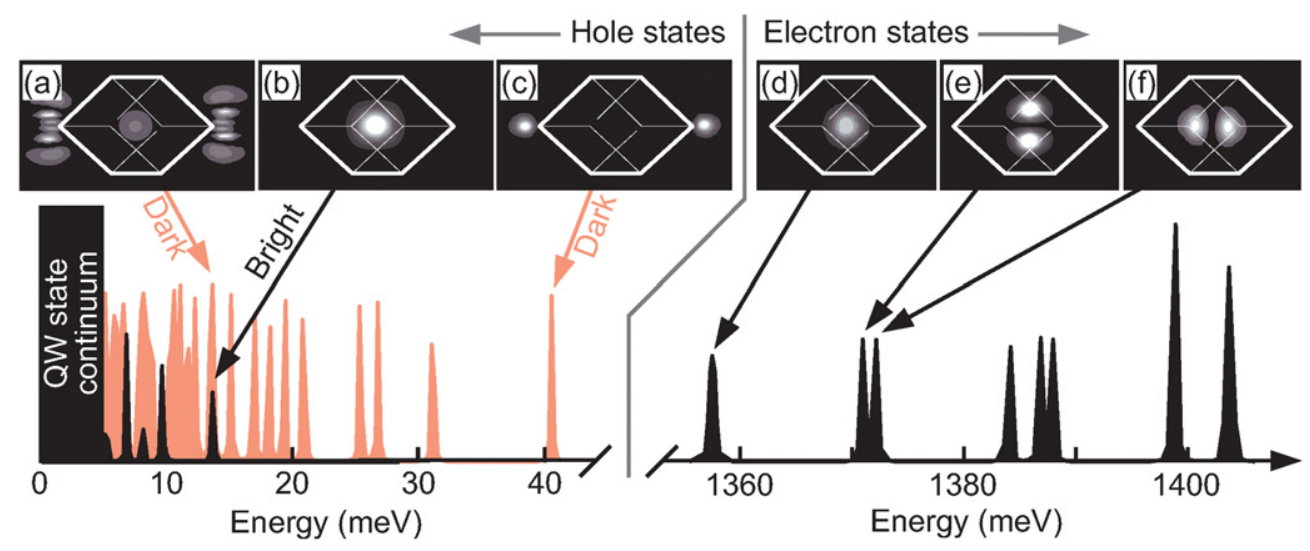

Figure 12. Density of hole and electron states, including states confined to the DP and PEP minima. The curve in the background is the total density of hole states, while the foreground (black) curve corresponds to the DOS of carriers confined to the DP minima only, i.e. the optically active states. Note that the two curves coincide for electrons, because the electron states are all confined to the DP minimum. The top panels $(a)-(f)$ show the probability densities of selected states with respect to the position of the InP island.

states, of which several are more strongly confined than the hole ground-state $\left|h_{0}\right\rangle$ of the DP minimum (figure 12(b)). The confinement energy of the PEP ground-state $\left|p_{0}\right\rangle$ (figure 12(c)) is several tens of meV larger than that of $\left|h_{0}\right\rangle$. Note that the probability density of some hole states is distributed over both the PEP minima and the DP minimum (figure 12(a)). These states do have a non-zero wave function overlap with $\left|h_{0}\right\rangle$ and with the $\left|e_{i}\right\rangle$ electron states.

The influence of the InP island geometry can be studied by comparing electronic structures of SIQD induced by both an angular (see figure 1 and [11]) and an axisymmetric InP island (like the one used in [13]). Figure 13 shows the electron DOS in a SIQD beneath $(a)$ an axisymmetric and $(b)$ an angular InP stressor island. It also shows the influence of the PEP on the particular SIQD geometries. The solid lines and the filled curves correspond to electron structure calculations including and excluding the PEP, respectively.

The effect of the PEP on the electron ground-state $\left|e_{0}\right\rangle$ is small for both geometries; however, it increases for the excited states as a result of the increased lateral wave function spreading. The PEP lowers the level degeneracy and splits the DOS into several smaller peaks. In analogy, any deviation from axisymmetric island geometry lowers the level degeneracies and generates new peaks in the DOS. However, the effect of both the island geometry and the PEP decreases very effectively as a function of the distance between the InP island and the QW. The DOS of figures 12 and 13 is represented in terms of Gaussian peaks of finite width $(\Gamma=0.5 \mathrm{meV})$ for clarity.

\subsection{The influence of an external electric field}

An electric field separates electron and hole states spatially and shifts the eigenenergies as a result of a tilted QD confinement potential. Electric fields applied to QD confined semiconductor systems (i.e. the QCSE) cause large changes in the PL energies and recombination intensities by polarizing the confined electron hole systems.

Strain-induced quantum dots are very sensitive to an electric field as a result the very shallow confinement potential of holes. We have simulated the QCSE for SIQD, using the 


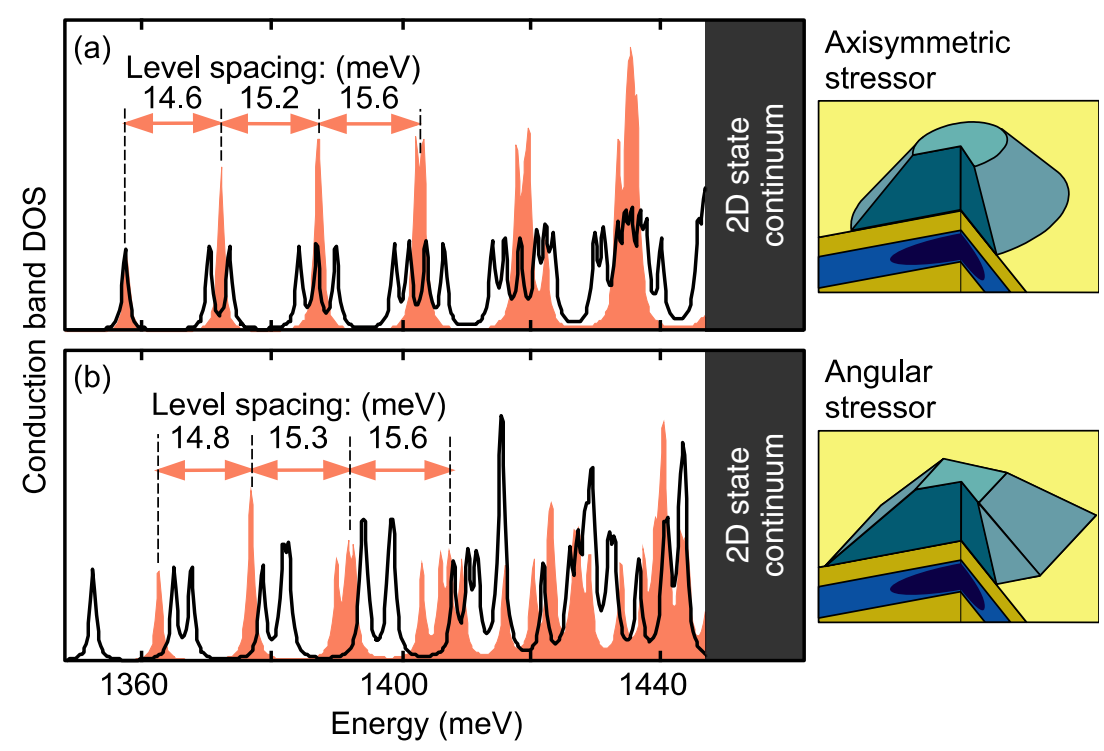

Figure 13. The density of electron states in the case of $(a)$ an axisymmetric and $(b)$ an angular InP stressor island. The insets show the corresponding island geometries. The solid lines and the filled curves correspond to simulations which include and exclude the PEP, respectively.

conventional eight-band EFA model. Figure 14 shows the SP energies of $(a)$ electrons and (b) holes for an electric field along [ 110$]$ (solid lines) and along [ $\left.\begin{array}{lll}1 & 1 & 0\end{array}\right]$ (dashed lines). The electron energies are in practice unaffected by the electric field. This is well in line with the parabolic confinement potential of the electrons. The Stark shift of the hole energies is a few meV for $\boldsymbol{E} \|\left[\begin{array}{lll}1 & 1 & 0\end{array}\right]$. The hole energies are fairly insensitive to the electric field along $\left[\begin{array}{lll}1 & 1 & 0\end{array}\right]$ whereas the hole probability densities move very easily along the electric field when $\boldsymbol{E} \|\left[\begin{array}{lll}1 & 1 & 0\end{array}\right]$ direction (see figure 15). This is due to the weak confinement potential and the attractive PEP along the [ $\left.\begin{array}{lll}1 & 1 & 0\end{array}\right]$ diagonal of the SIQD. This is also seen in the recombination strength, which fades out, for $E_{\left[11_{10}\right.} \gtrsim 0.2 \mathrm{mV} \mathrm{nm}^{-1}$, for the ground-state to ground-state transition $\left(\Psi_{\mathrm{e} 0} \rightarrow \Psi_{\mathrm{h} 0}\right)$.

The Stark shift of the excitonic ground-state recombination energy $E_{\mathrm{eh}}$ can be written as a function of the field strength $\xi$ using

$$
\Delta E_{\mathrm{eh}}=\mu \xi+\alpha \xi^{2},
$$

where $\mu$ and $\alpha$ are the components of the permanent dipole moment and the polarizability, respectively, in the direction of the electric field. In the single-particle approximation (figure 14 and figure $16(a)$ ) we obtain $\alpha_{x}=-0.451 \mathrm{meV}\left(\mathrm{kV} \mathrm{cm}^{-1}\right)^{-2}$ for $\boldsymbol{\xi} \|$ [1 10 ] and $\alpha_{y}=-0.065 \mathrm{meV}\left(\mathrm{kV} \mathrm{cm}^{-1}\right)^{-2}$ for $\boldsymbol{\xi} \|\left[\begin{array}{lll}\overline{1} & 1 & 0\end{array}\right]$. The simulated confinement potential was symmetric with respect to the $x$ and $y$ axes. The permanent dipole was, therefore, zero $\mu_{x}=\mu_{y}=0$.

The excitonic carrier-carrier interaction is enhanced in quantum confined carrier systems, in particular in zero-dimensional QD (see section 4). In order to estimate its effect on the QCSE, we have calculated the recombination energies including the electron-hole Coulomb interaction using first-order perturbation theory. The electron-hole Coulomb energy is given by

$C_{\mathrm{e} i, \mathrm{~h} j}=-\frac{e^{2}}{4 \pi \varepsilon_{0}}\left\langle\Psi_{\mathrm{e} i}\left(\boldsymbol{r}_{\mathrm{e}}\right)\left|\left\langle\Psi_{\mathrm{h} j}\left(\boldsymbol{r}_{\mathrm{h}}\right)\left|\frac{1}{\varepsilon_{r}\left(\boldsymbol{r}_{\mathrm{e}}, \boldsymbol{r}_{\mathrm{h}}\right)\left|\boldsymbol{r}_{\mathrm{e}}-\boldsymbol{r}_{\mathrm{h}}\right|}\right| \Psi_{\mathrm{h} j}\left(\boldsymbol{r}_{\mathrm{h}}\right)\right\rangle\right| \Psi_{\mathrm{e} i}\left(\boldsymbol{r}_{\mathrm{e}}\right)\right\rangle$, 
(a) Electron states

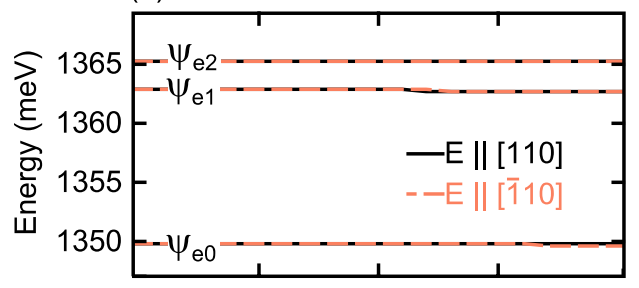

(b) Hole states

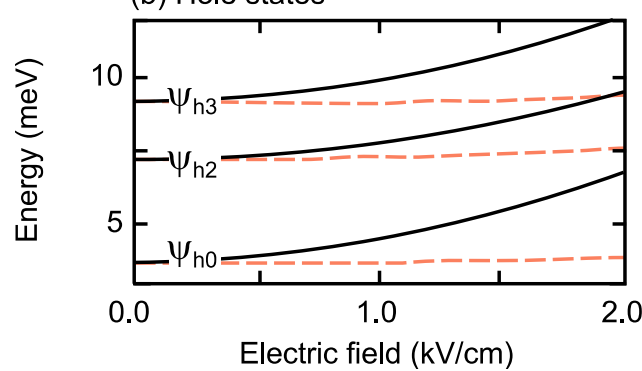

(c) PL energy

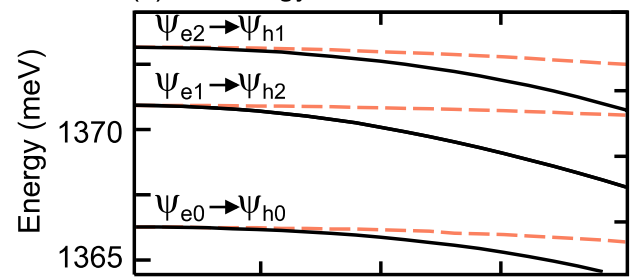

(d) PL Intensity

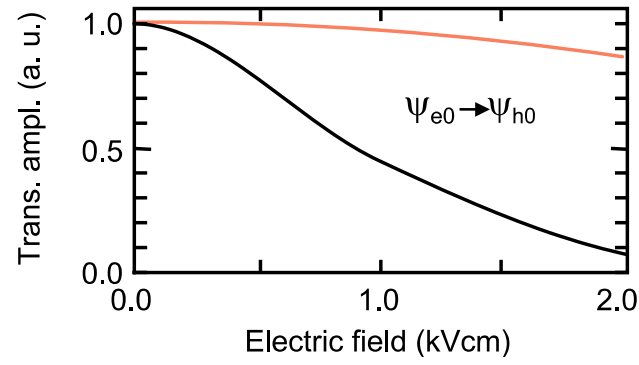

Figure 14. The energies of the $(a)$ electron and $(b)$ hole states as a function of electric field in the $\left[\begin{array}{lll}1 & 1 & 0\end{array}\right](-)$ and $\left[\begin{array}{lll}1 & 1 & 0\end{array}\right]\left(---_{-}\right)$directions. $(c)$ shows the energies of the dominant PL peaks and $(d)$ shows the strength of the ground-state PL as a function of the electric field. These curves were calculated in a single-particle approximation, omitting any particle-particle interaction.

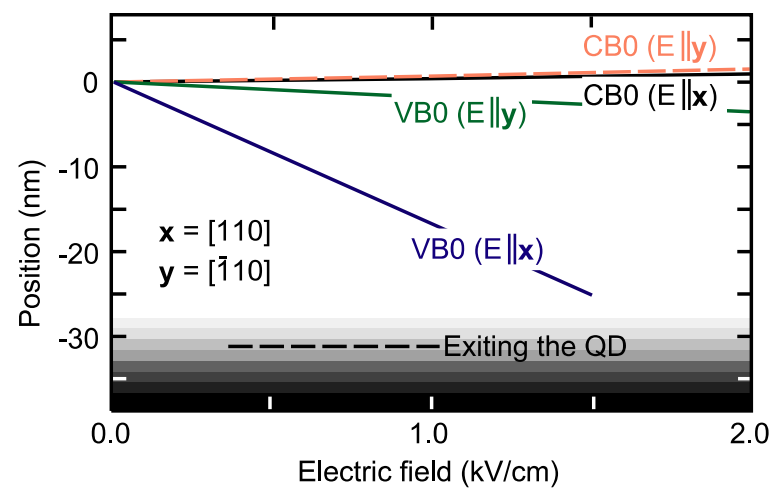

Figure 15. The shift of the probability densities of the electron and hole DP ground-states as a function of the external electric field in the single-particle approximation.

where $\varepsilon_{r}$ is the permeability, $\Psi_{(\mathrm{e} i / \mathrm{h} j)}$ are the electron or hole single-particle wave functions and $\boldsymbol{r}_{\mathrm{e}}$ and $\boldsymbol{r}_{\mathrm{h}}$ are the spatial coordinates of the integral. The ground-state recombination energy is obtained in the first-order perturbation theory as

$$
E_{\mathrm{e} 0, \mathrm{~h} 0}=E_{\mathrm{e} 0}-E_{\mathrm{h} 0}+C_{\mathrm{e} 0, \mathrm{~h} 0} \text {. }
$$

The Coulomb interaction energy is negative for an exciton and inversely proportional to the spatial separation of the electron and hole. It gives rise to an attractive force between the electron and hole, which opposes the electric field-induced electron-hole separation. Figure 16 shows the recombination energy of the ground-state transition as a function of the electric field, using both a single-particle and an exciton description. The total Stark 

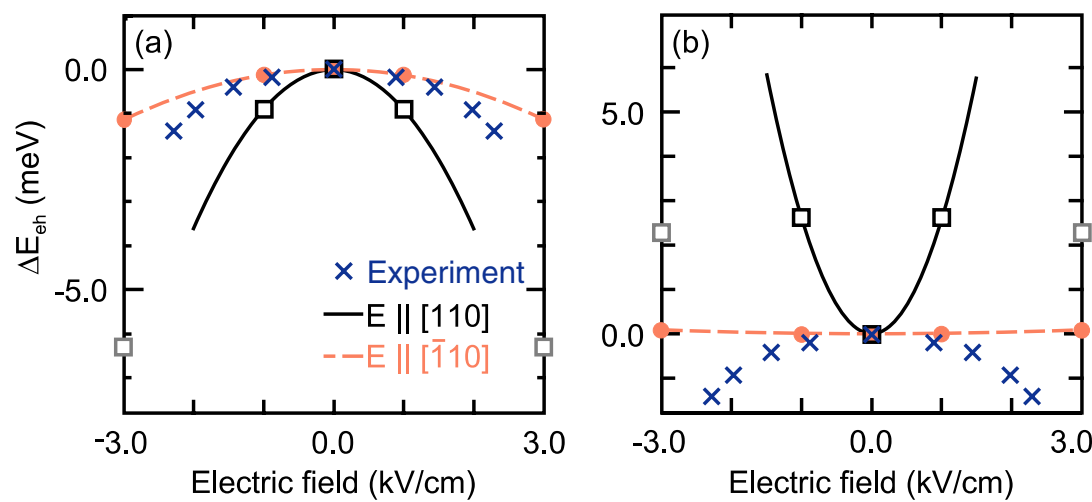

Figure 16. Stark shift of the ground-state recombination energy as a function of the electric field in the $\left[\begin{array}{lll}1 & 1 & 0\end{array}\right]$ direction (- ${ }_{-}$) and in the $\left[\begin{array}{lll}\overline{1} & 1 & 0\end{array}\right](---)$, calculated using $(a)$ a single-particle and (b) an exciton description of the electron and hole. The parabolas (solid lines) were only fitted for $|E| \leqslant 1 \mathrm{kV} \mathrm{cm}^{-1}$ since the holes escape the DP minimum for $|E|>1 \mathrm{kV} \mathrm{cm}^{-1}$ along [1 10 ]. The experimental data $(\times)$, were obtained from [91].

Table 3. Polarizability of strain-induced quantum dots, given in units of $\mathrm{meV}\left(\mathrm{kV} \mathrm{cm}^{-1}\right)^{-2}$. The electric field strength is denoted by $\xi$ (see (3.11)).

\begin{tabular}{|c|c|c|}
\hline Model & $\alpha$ for $\boldsymbol{\xi} \|\left[\begin{array}{lll}1 & 1 & 0\end{array}\right]$ & $\alpha$ for $\boldsymbol{\xi} \|\left[\begin{array}{lll}\overline{1} & 1 & 0\end{array}\right]$ \\
\hline Single-particle & -0.451 & -0.065 \\
\hline Exciton & 1.311 & 0.006 \\
\hline Experiment [91] & \multicolumn{2}{|c|}{$-0.13 \pm 0.02$} \\
\hline
\end{tabular}

shift (figure 16(b)), including the exciton interaction, becomes positive as a result of the attractive electron-hole Coulomb interaction and the weak Stark shift of the single-particle energies (figure 16(a)). The polarizability ( $\alpha$ in (3.11)) of the exciton is (using SI units) $\alpha_{x}=1.311 \mathrm{meV}\left(\mathrm{kV} \mathrm{cm}^{-1}\right)^{-2}$ and $\alpha_{y}=0.006 \mathrm{meV}\left(\mathrm{kV} \mathrm{cm}^{-1}\right)^{-2}$ (table 3).

The predicted polarizability is positive in the excitonic picture. However, this is in striking contrast to the experimental observations with SIQD [91] and more strongly confined QD [64, 92-96] and QW excitons [97]. We conclude that the single-particle picture is in better agreement with the experiments, indicating efficient screening of the electron-hole Coulomb interaction in SIQD. The result is surprising, although in agreement with the measurements of the magneto luminescence (see below) and the observed carrier density-independent QD luminescence line energies [98].

\subsection{The influence of an external magnetic field}

The magnetic field affects the intra-band coupling and modifies both diagonal and non-diagonal elements of the envelope function Hamiltonian (see, e.g. [81]). In an external homogeneous magnetic field the magnetic interaction will remove the (approximate) left-hand/right-hand symmetry around the growth axis. The magnetic confinement gives rise to a diamagnetic shift. The mesoscopic magnetic moment related to the motion of carriers around the symmetry axis of the QD and the spin magnetic moment, including both the atomic orbital angular momentum and electron spin, interact with the magnetic field. This gives rise to Zeeman and spin-splitting of energy levels, respectively. In very strong fields (well above $10 \mathrm{~T}$ ) the previous picture is modified by the formation of Landau levels. Because of the enhanced magnetic confinement 

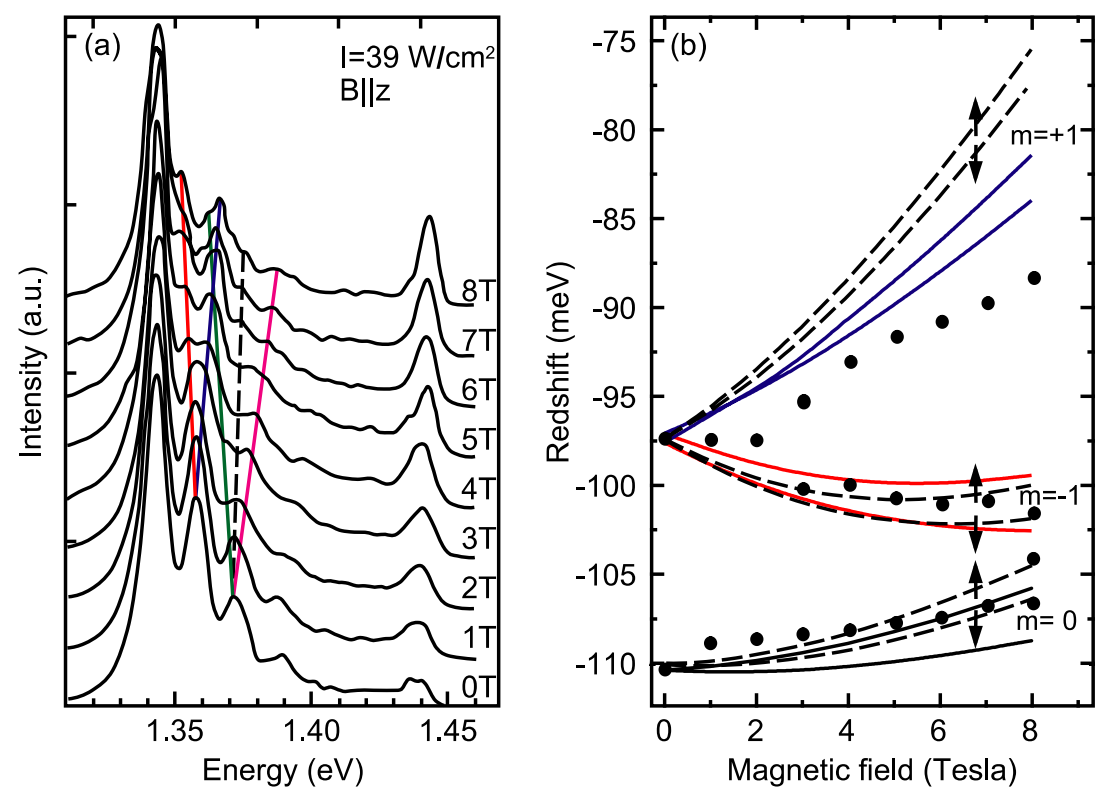

Figure 17. (a) Experimental magnetoluminescence spectra for $\boldsymbol{B} \|$ growth axis and $I_{\mathrm{exc}}=$ $39 \mathrm{~W} \mathrm{~cm}^{-2}$ [22]. The coloured lines connect split transitions arising from single bands at $0 \mathrm{~T}$. (b) Single-band (- - -) and four-band EFA ( - ) simulations of the diamagnetic shift and the Zeeman splitting compared with experiments $(-)$. The symbols $\uparrow / \downarrow$ correspond to the spin states of the single-band calculation and $m$ is the quantum number of the mesoscopic angular momentum [88].

the carrier-carrier correlation is enhanced in high magnetic fields. All these effects have been observed experimentally and analysed theoretically $[22,88,99,100]$. The theoretical analyses were based on an axisymmetric four-valence-band EFA model and a parabolic EFA model of the conduction band. The PEP was not included in these calculations. The theoretical results were, nevertheless, in fair agreement with experiments.

Figure 17(a) shows experimental $\mathrm{CW}$ magnetoluminescence spectra with the magnetic field aligned in parallel with the growth axis of the structure [22]. The zero-field PL spectrum exhibits four well-resolved peaks resulting from the radiative recombination of carriers from the four lowest QD energy levels. The ground-state PL peak is blue-shifted with increasing field strength resulting from the diamagnetic effect. The higher PL peaks, corresponding to the excited QD states, are broadened and split by the magnetic field into two or more peaks of different amplitudes and widths. This is clearly observable in figure $17(a)$ and indicated by the guiding lines for the first and second excited PL peaks. The diamagnetic shift is caused by a Hamiltonian element proportional to $B^{2}$. In the parabolic-band approximation the diamagnetic shift is given by

$$
\Delta E_{\mathrm{D}}=\frac{e^{2}}{8 m_{0}}\left(\frac{\left\langle r^{2}\right\rangle^{\mathrm{e}}}{m_{\mathrm{e}}^{*}}+\frac{\left\langle r^{2}\right\rangle^{\mathrm{hh}}}{m_{\mathrm{hh}}^{*}}\right) B^{2},
$$

where the expectation values $\left\langle r^{2}\right\rangle^{\mathrm{e}}$ and $\left\langle r^{2}\right\rangle^{\mathrm{hh}}$ are calculated using electron (e) and hole (hh) wave functions, which include the effect of the magnetic field. The influence of the magnetic field on the wave functions is, however, very small at low field strengths and the diamagnetic shift $\Delta E_{\mathrm{D}}$ is, therefore, quadratic in $B$. At higher field strengths the magnetic field will reduce the expectation values of $r^{2}$ for both electron and hole wave functions. As a consequence the diamagnetic shift becomes linear in $B$ for strong magnetic fields. 
The Zeeman effect is seen in figure 17 as a splitting of single spectral lines into several components, caused by interaction between the mesoscopic magnetic momentum and the magnetic field. This removes the degeneracy of the $L_{z}= \pm m \hbar$ states. The parabolic and axisymmetric SIQD model gives transparent physical insight into the Zeeman effect (see section 1.3). The mesoscopic orbital angular momentum is the QD analogue to the atomic orbital angular momentum and is caused by the motion of carriers around the symmetry axis of the SIQD. The magnitude of this moment is given by $\mu=\mu_{\mathrm{B}} m / m^{*}$, where $\mu_{\mathrm{B}}=e \hbar / 2 m_{0}$ is the Bohr magneton, $m$ is the angular quantum number and $m^{*}$ is the effective electron or hole mass. The splitting $\Delta E_{\mathrm{D}}$ of a luminescence line is thereby obtained by adding the angular moments of the recombining electron-hole pair, multiplied by the field strength

$$
\Delta E_{\mathrm{D}}=\mu_{\mathrm{B}}\left(\frac{1}{m_{\mathrm{e}}^{*}}+\frac{1}{m_{\mathrm{h}}^{*}}\right) m B .
$$

The Zeeman splitting is one order of magnitude larger than the spin-splitting $\left(\Delta E_{\mathrm{D}} \approx 20 \mathrm{meV}\right.$ and $\Delta E_{\mathrm{s}} \approx 2 \mathrm{meV}$ for $m=1$ at $B=8 \mathrm{~T}$ ), mainly because of the effective masses in the denominators of (3.15). This splitting has been indicated in figure 17(b) by adding upward and downward arrows to the spin-split states.

Figure 17(b) shows the calculated and measured shifts of the recombination energies for the $1 \Sigma \rightarrow 1 \Sigma(m=0)$ and $1 \Pi \rightarrow 1 \Pi(m= \pm 1)$ transitions [88]. The parabolic-band EMA result is shown by dashed lines and is based on a lateral $\mathrm{HH}$ mass of $m_{\mathrm{hh}}^{*}=1 /\left(\gamma_{1}+\gamma_{2}\right)$. The EMA calculations reproduce successfully both the Zeeman splitting and the diamagnetic shift, confirming that the magnetic quantum number $m$ of the electron and hole states is approximately a 'good quantum number'. The magnetic dispersion, predicted by the confined exciton model [101], is, on the contrary, in clear disagreement with the experiments.

The effect of the valence band mixing on magnetoluminescence has been studied using an axisymmetric four-band EFA model [88]. These results are shown in figure 17(b) by solid lines. The inclusion of the band coupling does give a much better agreement with the experimental Zeeman splitting. A more extensive study of diamagnetic and Zeeman effects in SIQD was published by Rinaldi et al in [99] and supports all the conclusions given above.

In very high magnetic fields the above picture is drastically changed by the formation of Landau levels. Cingolani et al have studied electron-hole correlation under high magnetic fields (up to 45 T) [100]. Figure 18 shows a comparison of the experiments with Hartree-Fock calculations. The experimental data corresponds to the PL spectra of figure 5, reproduced from [100]. The formation of Landau levels, under very strong magnetic fields (for $B>10 \mathrm{~T}$ ), is clearly observable. The carrier-carrier correlation effects are greatly enhanced as a result of the increased magnetic squeezing of the carriers [100]. This enhancement of the correlation is mainly due to enhanced electron-electron and hole-hole exchange energy. The electron-hole exchange interaction is presumably small.

\section{Electronic structure II-many-particle description}

One of the remaining unsolved puzzles of SIQD is the unexpectedly good agreement between single-particle models and experiments (see, e.g. [23] and references therein). The mean-field approximation (also called the HF or self-consistent-field method) [102-104] can be used to include the direct and exchange Coulomb interaction to the lowest order. However, the HF method omits the correlation energy of the Coulomb interaction. This shortcoming becomes potentially significant for QD, which contain both electrons and holes, with repulsive and attractive carrier-carrier interactions. 

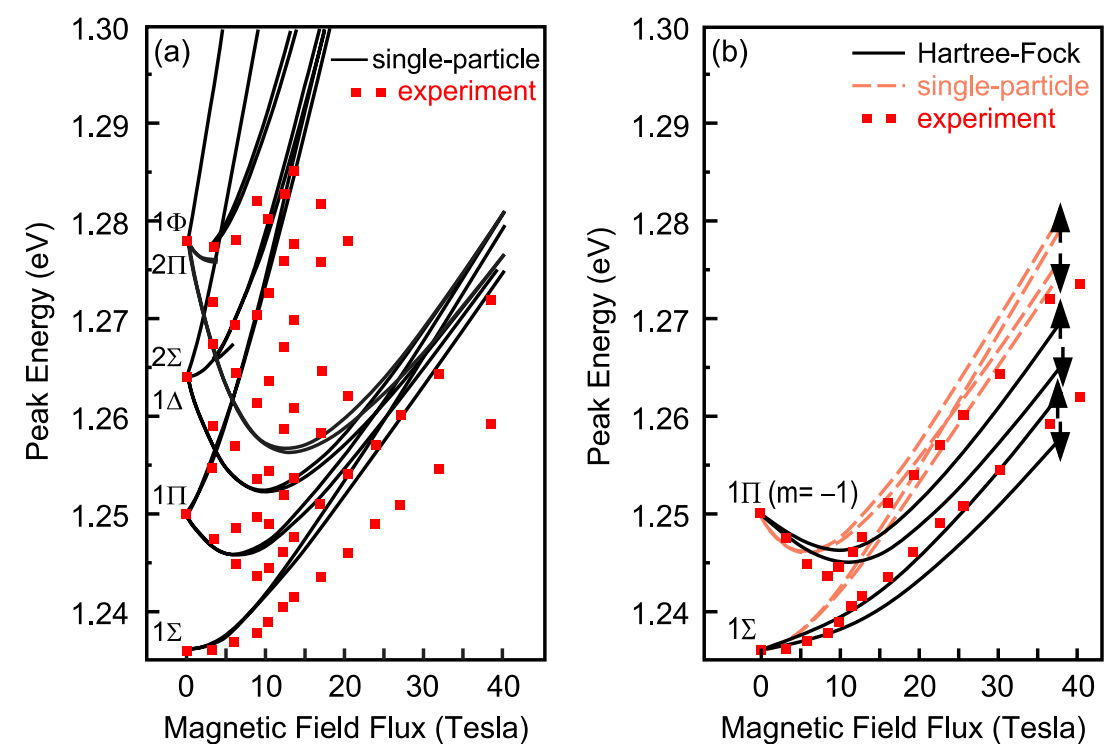

Figure 18. (a) Experimental ( $\boldsymbol{\square}$ ) [100] and calculated (-) single-particle magnetic field dependence of the ground $1 \Sigma$ and excited $1 \Pi, 2 \Sigma, 2 \Pi$ QD states. $(b)$ Experimentally measured (ש) and theoretically predicted diamagnetic shift of the ground Landau level (consisting of the $1 \Sigma$ and $m<0,1 \Pi$ transitions). The theoretical shifts were calculated in the single-particle approximation (- - -) and using the full Hartree-Fock model ( - ), which accounts for the electron-hole correlation effects. The symbols $\uparrow$ and $\downarrow$ on the curves indicate the spin orientation.

Braskén and co-workers have modelled SIQD using an effective-mass CI approach which also includes the Coulomb-correlation effects [43,105-107]. More recently, Vänskä et al have reported on the modelling of multi-exciton states in SIQD using the coupled cluster approach [108]. This technique is to be considered as an effective approximation of the full CI. Related CI approaches have also been used for the analysis of other types of QD (see, e.g. [109-113]). Regrettably, to make the calculations computationally feasible, the many-body calculations of Braskén et al had to be carried out using a two-band EMA. Nevertheless, their work gives valuable insight into the influence of carrier-carrier correlation in SIQD.

In the CI scheme the many-particle eigenstate is written as a linear combination of single configuration wave functions. The single configuration eigenstates, also called Slater determinants, are antisymmetrized products of single-particle $\mathrm{SpO}$ of electrons and holes [105,114]. The SpO are commonly calculated with the HF technique [115]. The coefficients of the expansion are calculated by an iterative diagonalization of the many-particle Hamiltonian.

In the second quantization, the many-body Hamiltonian can be written as the sum of the SP energies $H^{\text {free }}$ and the carrier-carrier correlation terms $H^{\mathrm{ee}}, H^{\mathrm{hh}}$, and $H^{\mathrm{eh}}$ :

$$
H=H^{\text {free }}+H^{\mathrm{ee}}+H^{\mathrm{hh}}+H^{\mathrm{eh}} .
$$

The different terms of the Hamiltonian are given by

$$
\begin{aligned}
& H^{\mathrm{free}}=\sum_{i j} h_{i j}^{\mathrm{e}} c_{i}^{\dagger} c_{j}+\sum_{r s} h_{r s}^{\mathrm{h}} h_{r}^{\dagger} h_{s}, \\
& H^{\mathrm{ee}}=\frac{1}{2} \sum_{i j k l} V_{i j, k l} c_{i}^{\dagger} c_{k}^{\dagger} c_{l} c_{j},
\end{aligned}
$$




$$
\begin{aligned}
& H^{\mathrm{hh}}=\frac{1}{2} \sum_{r s t u} V_{r s, t u} h_{r}^{\dagger} h_{t}^{\dagger} h_{u} h_{s}, \\
& H^{\mathrm{eh}}=-\sum_{i j r s} V_{i j, r s} c_{i}^{\dagger} c_{j} h_{r}^{\dagger} h_{s},
\end{aligned}
$$

where $c_{i}^{\dagger}\left(h_{i}^{\dagger}\right)$ is the electron (hole) creation operator for the single-particle spin orbital $i$ and $c_{i}\left(h_{i}\right)$ is the electron (hole) annihilation operator of the spin orbital $i$. The SP energies contain the QD kinetic energy and confinement potential $V_{\mathrm{QD}}^{(\mathrm{e} / \mathrm{h})}$, i.e.

$$
h_{i j}^{(\mathrm{e} / \mathrm{h})}=\int \mathrm{d} \boldsymbol{x} \psi_{i}^{*}(\boldsymbol{x})\left[-\frac{\hbar^{2}}{2 m_{0}} \nabla \frac{1}{m_{(\mathrm{e} / \mathrm{h})}^{*}} \nabla+V_{\mathrm{QD}}^{(\mathrm{e} / \mathrm{h})}(\boldsymbol{r})\right] \psi_{j}(\boldsymbol{x}),
$$

which includes both the spatial integration over $r$ and the summation over the spin $m_{\mathrm{s}}$ and $m_{(\mathrm{e} / \mathrm{h})}^{*}$ is the relative effective mass of either an electron or a hole. Braskén et al calculated the SpO wave functions $\psi_{i}(\boldsymbol{x})$ using a two-band model [105]. More advanced techniques, based on a pseudopotential description of the band structure, have been used for the SpO of other types of QD $[113,116]$. The Coulomb matrix elements $V_{i j, k l}$ of (4.2) are given by

$$
V_{i j, k l}=\int \mathrm{d} \boldsymbol{x} \int \mathrm{d} \boldsymbol{x}^{\prime} \psi_{i}^{*}(\boldsymbol{x}) \psi_{k}^{*}\left(\boldsymbol{x}^{\prime}\right) \frac{e^{2}}{4 \pi \epsilon\left|\boldsymbol{r}-\boldsymbol{r}^{\prime}\right|} \psi_{j}(\boldsymbol{x}) \psi_{l}\left(\boldsymbol{x}^{\prime}\right) .
$$

The electron-hole exchange terms (Coulomb matrix elements with a wave function overlap integral between an electron and a hole state) are small as a result of the wide band gap [117] and have consequently been excluded from the many-particle Hamiltonian in (4.1).

In this review we will mainly discuss charge-neutral QD carrier configurations. This is motivated by experimental QD spectroscopy $[4,118]$ and is in accordance with the work of Braskén et al [105]. For configuration interaction calculations of charged SIQD see [119] by Corni et al. The wave function $|N, k\rangle$ of a neutral state, composed of $N$ electrons and $N$ holes, can be expanded in a CSF basis, i.e. a linear combination of Slater determinants:

$$
|N, k\rangle=\sum_{I=\left(i_{1}, \ldots, i_{N} ; r_{1}, \ldots, r_{N}\right)} C_{I}^{(N, k)} c_{i_{1}}^{\dagger}, \ldots, c_{i_{N}}^{\dagger} h_{r_{1}}^{\dagger}, \ldots, h_{r_{N}}^{\dagger}|0\rangle
$$

where $|0\rangle$ is the vacuum state of an empty QD. The energy and the coefficients $C_{I}^{(N, k)}$ of the CSF state are determined by diagonalizing the many-body Hamiltonian in the CSF basis.

\subsection{Hartree-Fock approximation}

In the HF approximation only one, symmetry-adopted, Slater determinant is used. The electron and hole wave functions (orbitals) of the configuration studied ( $N$ electrons and $N$ holes) are calculated iteratively. In the HF approximation, one calculates the wave functions of the $i$ th particle, including the effect of the other $2 N-1$ particles, on the basis of their orbitals of the previous iteration. The HF equation of the $i$ th one electron (and hole) spin-orbital is given by

$$
\hat{f}\left|\psi_{i}\right\rangle \equiv \hat{h}^{\mathrm{e} / \mathrm{h}}\left|\psi_{i}\right\rangle+\sum_{j \neq i}^{2 N}\left\langle\psi_{j}|V| \psi_{j}\right\rangle\left|\psi_{i}\right\rangle-\sum_{j \neq i}^{2 N}\left\langle\psi_{j}|V| \psi_{i}\right\rangle\left|\psi_{j}\right\rangle=\epsilon_{i}\left|\psi_{i}\right\rangle,
$$

where the summations are performed over all other electrons and holes $(j \neq i), \hat{f}$ is the Fock operator and $V$ is the Coulomb interaction. The electron and hole orbitals are product states of the spatial function $\chi_{i}(\boldsymbol{r})$ and the spin function $(\alpha, \beta)^{\mathrm{T}}$

$$
\psi(\boldsymbol{r})=\chi(\boldsymbol{r})\left(\begin{array}{l}
\alpha \\
\beta
\end{array}\right) \text {. }
$$


The spatial part of the orbitals is commonly expanded in terms of a set of basis functions $\chi_{i}(\boldsymbol{r})=\sum_{\nu} c_{v i} \phi_{\nu}$. In QD simulations, one uses a basis set of either numerical SpO eigenstates (see, e.g. $[116,120]$ and references therein) or computationally effective analytic functions.

Braskén et al used axisymmetric Gaussian basis functions for the carrier wave functions in SIQD [105]. This was motivated by the nearly parabolic confinement potential of SIQD and by computational benefits permitting simulations of up to $\sim 10$ electron-hole pairs. Braskén et al also adopted the restricted HF approximation [105], i.e. the same spatial dependence of the $\mathrm{SpO}$ was used for electrons (and holes) with both spin $\alpha$ and $\beta$. By substituting the basis function expansion in (4.9) we obtain the following matrix equation

$$
\left(\begin{array}{cc}
\boldsymbol{F}^{\mathrm{e}} & 0 \\
0 & \boldsymbol{F}^{\mathrm{h}}
\end{array}\right)\left(\begin{array}{l}
c^{\mathrm{e}} \\
c^{\mathrm{h}}
\end{array}\right)=E\left(\begin{array}{ll}
\boldsymbol{S} & 0 \\
0 & S
\end{array}\right)\left(\begin{array}{l}
c^{\mathrm{e}} \\
c^{\mathrm{h}}
\end{array}\right) \text {. }
$$

The electron and hole elements of the Fock matrix $\boldsymbol{F}$ are given by

$F_{i j}^{\mathrm{e}}=h_{i j}^{\mathrm{e}}+\sum_{k l} P_{k l}^{\mathrm{e}}\left(\langle i j|V| k l\rangle-\frac{1}{2}\langle i l|V| k j\rangle\right)-\sum_{p q} P_{p q}^{\mathrm{h}}\langle i j|V| p q\rangle$,

$F_{p q}^{\mathrm{h}}=h_{p q}^{\mathrm{h}}+\sum_{r s} P_{r s}^{\mathrm{h}}\left(\langle p q|V| r s\rangle-\frac{1}{2}\langle p s|V| r q\rangle\right)-\sum_{i j} P_{i j}^{\mathrm{e}}\langle p q|V| i j\rangle$,

and the elements of the overlap matrix are given by $S_{i j}=\left\langle\phi_{i} \mid \phi_{j}\right\rangle$ and $\langle i j|V| k l\rangle=V_{i k, j l}$ are the Coulomb interaction terms between the product states $\left|\phi_{i}\right\rangle\left|\phi_{j}\right\rangle$ and $\left|\phi_{k}\right\rangle\left|\phi_{l}\right\rangle$ [see (4.7)]. The density matrix $\boldsymbol{P}$ is defined as the sum over the electron and hole orbitals; $P_{i j}=\sum_{\nu} n_{\nu} c_{v i} c_{j v}$, where $n_{v}$ is the occupation number $(n \in\{0,1,2\})$. The iterative algorithm of the HF scheme was described in detail in [107].

\subsection{Configuration interaction scheme}

The CI scheme accounts for the carrier-carrier correlations through the inclusion of excited many-body states, in addition to the HF ground-state. The CI many-body eigenstates are formed from linear combinations of Slater determinants (i.e. CSF) [121],

$$
|\Psi\rangle=c_{0}\left|\Psi_{0}\right\rangle+\sum_{r a} c_{a}^{r}\left|\Psi_{a}^{r}\right\rangle+\sum_{\substack{a<b \\ r<s}} c_{a b}^{r s}\left|\Psi_{a b}^{r s}\right\rangle+\ldots,
$$

where $\left|\Psi_{0}\right\rangle$ is the HF ground-state and $\left|\Psi_{a}^{r}\right\rangle$ is a singly excited Slater determinant where one electron (or hole) has been excited from the occupied orbit $\psi_{a}$ to an empty (virtual) orbit $\psi_{r}$. The doubly excited Slater determinant $\left|\Psi_{a b}^{r s}\right\rangle$ is obtained from $\left|\Psi_{0}\right\rangle$ by exciting one particle from $\psi_{a}$ to $\psi_{r}$ and another from $\psi_{b}$ to $\psi_{s}$. The set $\left\{\left|\Psi_{0}\right\rangle,\left|\Psi_{a}^{r}\right\rangle,\left|\Psi_{a b}^{r s}\right\rangle, \ldots\right\}$ of all possible $N$-electron-hole Slater determinants forms a complete basis set for the description of any $N$ pair wave functions. The coefficients of (4.14) are determined by minimizing the total energy $\langle\Psi|H| \Psi\rangle$ and keeping $|\Psi\rangle$ normalized [122]. In the FCI the energy minimization is performed using the complete set of Slater determinants, without truncation. When studying large carrier populations, in practice one is forced to truncate the set of Slater determinants. Braskén et al used a truncated CI method when studying systems of more than four electron-hole pairs. A more detailed description of the CI technique, applied to SIQD, can be found in [104,114, 123].

\subsection{Correlation energy}

Braskén et al calculated the addition/subtraction spectrum [123] (also called charging energy) for axisymmetric SIQD. That is the energy $\mu(N)$ which is needed to add an electron-hole pair 


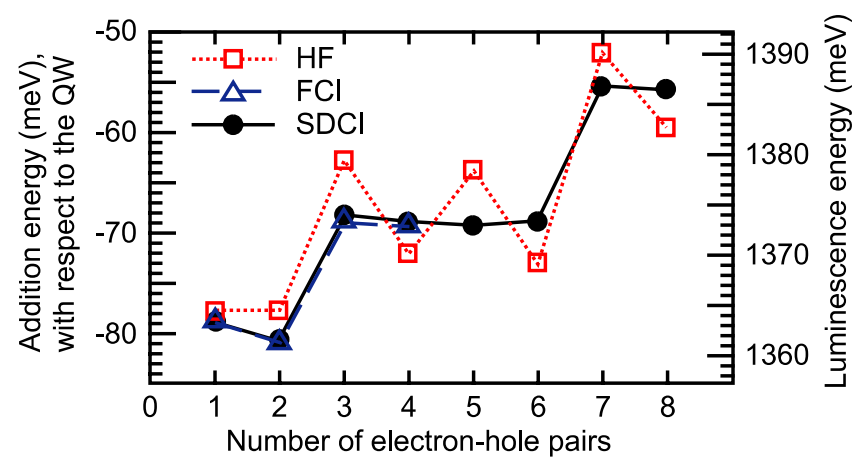

Figure 19. Addition energy per electron-hole pair calculated at the HF level ( $\square$ ), using the FCI approach $(\triangle)$ and using the SDCI $(\bullet)$. Reported by Braskén et al in [123].

to a QD which is already occupied by $N-1$ electron-hole pairs. This is the same energy that one gains when removing one electron-hole pair from the highest occupied QD SpO. The addition/substraction energy is given by the energy difference between the total many-body energies of having $N$ and $N-1$ electron-hole pairs on the QD:

$$
\mu(N)=E(N)-E(N-1) .
$$

Figure 19 shows the addition energies calculated at the HF level, using the SDCI and the FCI approach. A clear shell structure is seen in the spectrum as a result of the parabolic and axisymmetric geometry. The energy required to add the second electron-hole pair is only $2 \mathrm{meV}$ less than the addition energy of the first electron-hole pair [123]. This is far too small to be observed in standard PL experiments, but should be observable in single-QD spectroscopy (see figure 4). The addition energy of the third electron-hole pair exceeds that of the second by $10.5 \mathrm{meV}$. The addition energy of charged SIQD has been reported in [119].

The CI calculation of figure 19 can be compared with low-temperature luminescence spectra. The axis on the RHS represents the photoluminescence energy of the electronhole pair on the highest occupied levels as a function of the number of confined excitons (cf figure 4(a)). The axis on the LHS shows the photon energy with respect to the energy of the lowest QW recombination energy. The $\sim 10 \mathrm{meV}$ steps in figure 19 correspond to the energy separation of the luminescence peaks in figure $4(a)$. The theory is in good qualitative agreement with the experiment. Note, however, that the HF result would also be in good agreement with the experiment, if one averaged over the spin pairing energy [124] by an appropriate choice of HF basis functionals. This suggests that the correlation energy is rather small after all!

\subsection{Electron-hole spatial correlation}

The pair correlation is defined as [104]

$$
\rho_{2}\left(\boldsymbol{x}_{1}, \boldsymbol{x}_{2}\right)=N(N-1) \int \mathrm{d} \boldsymbol{x}_{3} \cdots \mathrm{d} \boldsymbol{x}_{N}\left|\Psi\left(\boldsymbol{x}_{1}, \boldsymbol{x}_{2}, \boldsymbol{x}_{3}, \ldots, \boldsymbol{x}_{N}\right)\right|^{2}
$$

and represents the probability of finding one particle at point $\boldsymbol{r}_{1}$ with spin $\sigma_{1}$, simultaneously with another particle at point $\boldsymbol{r}_{2}$ with spin $\sigma_{2}$. Figure 20 shows the two-particle correlation function of one electron-hole pair where the coordinate of the hole has been fixed in the centre of the QW plane at $\boldsymbol{r}_{\mathrm{h}}^{0}=[5,5,0 \mathrm{~nm}]$, i.e. $\rho_{2}\left(\boldsymbol{x}_{\mathrm{e}}\right)=\rho_{2}\left(\boldsymbol{x}_{\mathrm{e}}, \boldsymbol{x}_{\mathrm{h}}^{0}\right)=2\left|\Psi\left(\boldsymbol{x}_{\mathrm{e}}, \boldsymbol{x}_{\mathrm{h}}^{0}\right)\right|^{2}$. This quantity represents the electron distribution of a single electron-hole pair under the condition that the hole is located at $\boldsymbol{r}_{\mathrm{h}}^{0}$ (indicated by a small dot in figure 20). This is, therefore, also an indicator 

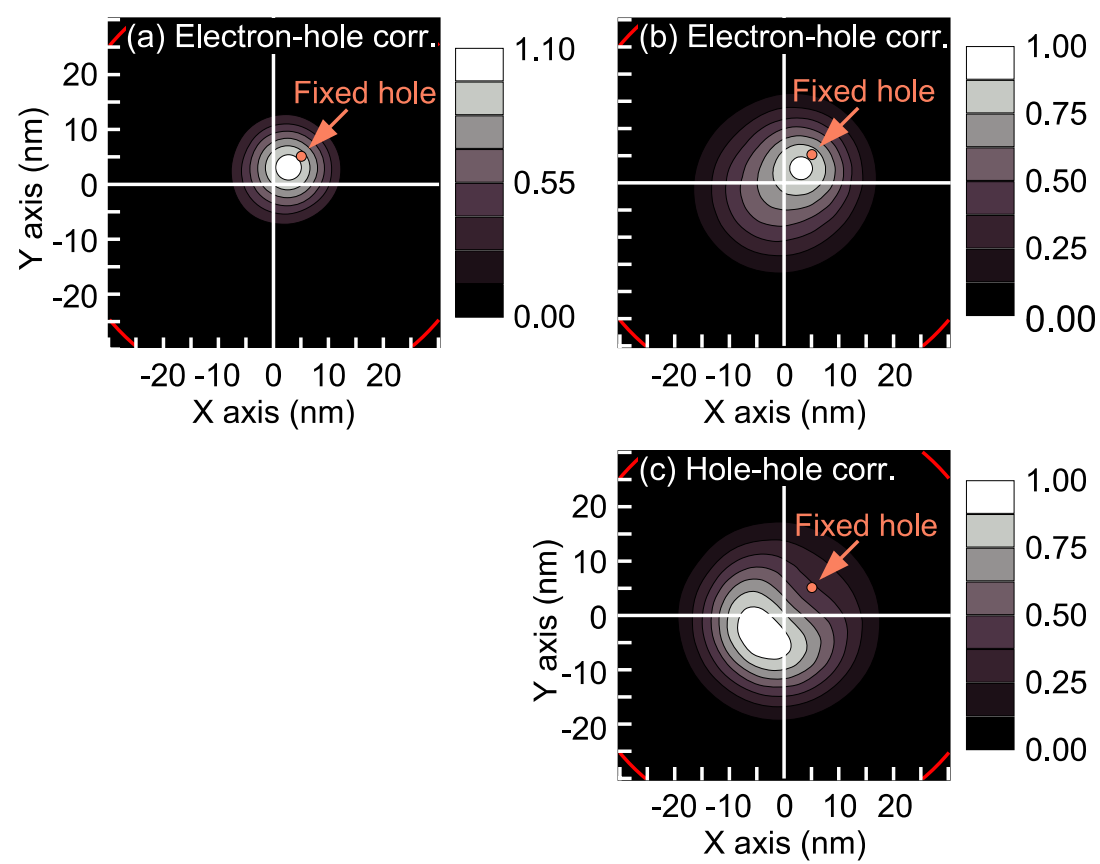

Figure 20. Spatial two-particle correlation function $\rho_{2}\left(\boldsymbol{r}_{\mathrm{e}}, \boldsymbol{r}_{\mathrm{h}}^{0}\right)$, calculated using the FCI technique for $(a)$ one electron-hole pair $(N=1),(b)$ electron-hole correlation function for $N=3$ and (c) hole-hole correlation function for $N=3$. Reprinted with permission from Braskén et al [104] (C) 2001 by the American Physical Society.

of whether the SIQD carriers form bound excitons or rather an electron-hole plasma. The radius of the stressor island (and of the effective confinement potential) was $R=40 \mathrm{~nm}$ in the calculation. The red lines in figure 20 show the circumference of the InP stressor island, inducing the QD.

In the HF approximation the electron distribution becomes independent of the hole position for one electron-hole pair. However, in the CI calculation the two-particle correlation function does not factorize as a result of the attractive electron-hole Coulomb interaction (which is only approximated in the pure HF approximation). The attractive correlation between the electron and the hole can also be seen in figure 20(a) [104]. The electron probability density is not centred around the QD symmetry axis. Instead, the electron follows the hole, forming a correlated electron-hole pair, i.e. an exciton.

Braskén et al found that two electrons (with antiparallel spins-spin singlet state) and two holes (also spin singlet) in an SIQD tend to form two strongly interacting excitons, i.e. a biexciton [104]. The two holes repel each other and the electrons are likely to be found in between the two holes. This is analogous to the $\mathrm{H}_{2}$ molecule.

The interpretation of the correlation function $\rho_{2}\left(\boldsymbol{x}_{1}, \boldsymbol{x}_{2}\right)$ is less trivial for QD occupied by many electron-hole pairs. In this case the correlation function represents the sum of the remaining electron and hole probability densities, because in this case the free coordinate can not be associated with only one particle. Figures $20(b)$ and $(c)$ show the electron-hole and hole-hole correlation for three electron-hole pairs $N=3[104,125]$. The holes are localized on opposite sides of the QD with the electrons distributed in between them, indicating the formation of a triexciton. 

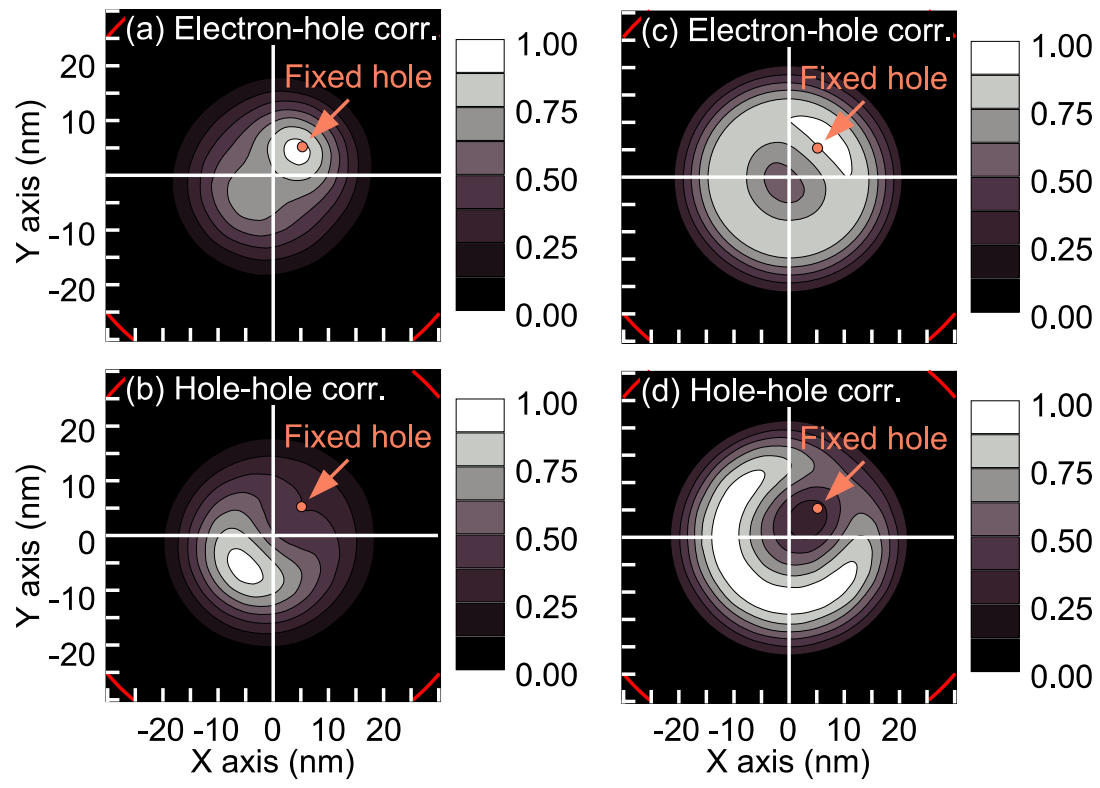

Figure 21. (a) Electron-hole and (b) hole-hole correlation functions for $N=4$, calculated using the FCI technique. $(c)$ Electron-hole and $(d)$ hole-hole correlation functions for $N=10$ calculated with the SDCI technique. Reprinted with permission from Braskén et al [104]. (C) 2001 by the American Physical Society.

In the case of $N=4$ (figures $21(a)$ and $(b)$ ) or more electron-hole pairs, the electron distribution is no longer associated with only one hole at $\boldsymbol{r}_{h}^{0}$. It is instead more distributed as a chemical bond between all holes. Both the electron and hole distributions become more spread out with increasing $N$ [104]. Figures 21(c) and $(d)$ show the correlation functions for $N=10$. At this point the electron and hole distributions are very ring-shaped. However, no type of crystalline ordering (Wigner crystal) of the carriers (as in purely electronic QD [126]) was found within the ring-shaped densities.

\section{Optical properties}

The intensity of spontaneous emission can be calculated in the electric dipole approximation by summing over the partial transition rates between all electron and hole states confined to an SIQD [63]. The PL intensity, as a function of the photon energy, is then given by [37,63]

$I_{\alpha}\left(E_{\hbar \omega}\right) \propto \sum_{i, f} \frac{g\left(E_{\hbar \omega}\right)}{E_{\hbar \omega}}\left|\left\langle\Psi_{\mathrm{e} i}\left|\boldsymbol{\alpha}^{*} \cdot \hat{\boldsymbol{p}}\right| \Psi_{\mathrm{h} f}\right\rangle\right|^{2} f_{\mathrm{e}}\left(E_{i}\right) f_{\mathrm{h}}\left(E_{f}\right) P\left\{\left[E_{\hbar \omega}-\left(E_{i}-E_{f}\right)\right], \Gamma\right\}$,

where $\alpha$ is the polarization vector of the photon field, $g(E)$ is the density of photon states $\left(g(E) \propto E^{2}\right.$ for a 3D system, i.e. in the absence of a photonic cavity) and $E_{i}$ and $E_{f}$ are the eigenenergies of the electron $\left|\Psi_{\mathrm{e} i}\right\rangle$ and hole $\left|\Psi_{\mathrm{h} f}\right\rangle$ states. In a typical experiment, the intensity of the PL, as a function of the photon energy, is given in arbitrary units. The factors that are independent of the state filling, temperature and photon energy are, therefore, not relevant when comparing theory and experiments. 
The experimentally observed inhomogeneous linewidth broadening of recombination energies $E_{\hbar \omega}=E_{i}-E_{f}$ is mainly due to a finite QD size distribution [23, 127, 128]. This effect has been approximated using the Gaussian broadening function $P(E, \Gamma)[129,130]$, where the inhomogeneous linewidth $\Gamma$ depends on the size variation of the QD. The Lorentz shaped homogenous linewidth broadening (caused by the finite lifetimes of the QD eigenstates) has a vanishing effect on the PL, which is already inhomogeneously broadened [63]. The homogenous linewidth broadening can, therefore, safely be neglected unless a single QD is analysed. Note that the effect of charge fluctuations on the recombination linewidths might also be important under high state filling. The temperature-dependent statistical distribution of electrons and holes under quasi-equilibrium conditions is described by the respective quasiFermi levels $E_{\mathrm{fe}}$ and $E_{\mathrm{fh}}$.

The matrix element $\left\langle\Psi_{\mathrm{e} i}|\hat{\boldsymbol{p}}| \Psi_{\mathrm{h} f}\right\rangle$ of the electric dipole (the electron-photon interaction) is given in the EFA by

$$
\left\langle\Psi_{\mathrm{e} i}|\hat{\boldsymbol{p}}| \Psi_{\mathrm{h} f}\right\rangle=\sum_{l m}\left[\left\langle F_{l}^{(\mathrm{i})}\left|\left\langle u_{l}|\hat{\boldsymbol{p}}| u_{m}\right\rangle\right| F_{m}^{(\mathrm{f})}\right\rangle+\delta_{l m}\left\langle F_{l}^{(\mathrm{i})}|\hat{\boldsymbol{p}}| F_{m}^{(\mathrm{f})}\right\rangle\right],
$$

where $F_{l}^{(\mathrm{i})}\left(F_{l}^{(\mathrm{f})}\right)$ are the envelope functions of the initial (final) state and $u_{l}$ are the Bloch functions. The first term on the RHS of (5.2) can be derived from the eight-band Hamiltonian [55]

$$
\left\langle u_{l}|\hat{\boldsymbol{p}}| u_{m}\right\rangle=\frac{m_{0}}{\hbar}\left\langle u_{l}\left|\nabla_{\boldsymbol{k}} H\right| u_{m}\right\rangle,
$$

and the second term is close to zero for any inter-band transition.

\subsection{Photoluminescence}

Figure 22(a) shows the PL of SIQD. The blue peaks show individual recombinations and their relative strength, which combine to form the total PL. The black line, simulating the PL of a large QD ensemble, was obtained by summing over the individual transitions, with an inhomogeneous broadening of $\Gamma=6 \mathrm{meV}$ (estimated on the basis of experimentally observed size distributions of InP stressor islands [23,127, 128]). Figure 22(b) shows the state filling of the radiative SIQD states in terms of the PL as a function of the number $N$ of electron-hole pairs. Figure 22(a) corresponds to zero temperature and complete population inversion (i.e. $f_{\mathrm{e}}=1$ and $f_{\mathrm{h}}=1$ for all electron and hole states, respectively), whereas figure $22(b)$ was computed for a fixed number of quasi-Fermi-distributed electrons and holes at a temperature of $T=10 \mathrm{~K}$. Increasing $N$ is comparable with increasing the optical pumping intensity. The results are in excellent agreement with the experimental data [4,22].

Characterization of the PL peaks according to the symmetry of the eigenstates involved is difficult because of the weak confinement and small energy spacing of the hole states (1$4 \mathrm{meV}$ ). Only the two lowest peaks (QD1 and QD2) are due to recombination between clearly confined electron and hole states. The higher PL peaks are due to recombination between a few confined electron states and several confined or quasi-confined hole states (i.e. the hole wave function are shared between the DP and PEP minima, with their energies merging with the energy continuum of the QW).

Figure 23(a) shows simulated PL spectra, averaged over the polarization, for an ensemble of SIQD together with an experimental luminescence spectrum [4]. The QD PL was calculated using both the angular stressor geometry shown in figure 1 and using an axisymmetric model (also discussed in section 3.4). All results are based on fully 3D simulations. However, in the axisymmetric model the SIQD was induced by an InP island in the shape of a truncated cone, with the same height, the same InP volume and the same bottom area as the angular island shown in figure 1 . The calculation of radiative transition energies and line strengths shows good 

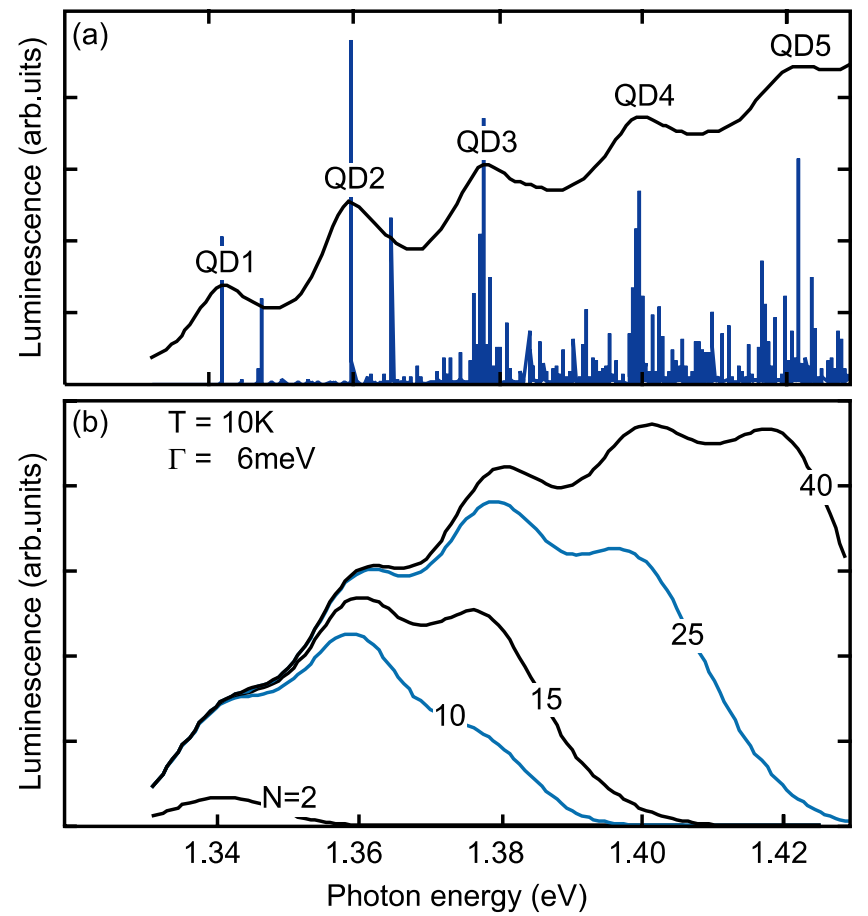

Figure 22. (a) PL of a SIQD ensemble, simulated by a Gaussian broadening (inhomogeneous linewidth broadening) of all individual transition amplitudes of a single QD simulation. The $\delta$ peaks show the transition amplitudes and position in energy of all electron-hole recombination processes. ( $b$ ) The PL intensity as a function of the number $N$ of quasi-Fermi distributed electronhole pairs in the radiative states of a SIQD.

agreement with the experimental intensity distribution and energy spacing of the luminescence peaks [4]. Figure 23 corresponds to zero temperature and complete population inversion (i.e. $f_{\mathrm{e}}=1$ and $f_{\mathrm{h}}=1$ for all electron and hole states, respectively). The simulated PL was normalized by equating the QD4 peak intensities to that of the corresponding experimental QD4 peak. The qualitative agreement between the simulations and the experiments is very good.

The discrepancies between the two numerical simulations in figure 23( $a)$ are small. The exact geometry of the stressor island affects the electron and hole states of the SIQD, mainly through the PEP and in the vicinity of the edges of the island (see figures 7 and 8). Figure 23(b) shows the effect of the PEP on the QD PL of a SIQD, induced by the axisymmetric stressor island. The effect of the PEP, on the most strongly confined electron and hole states is small (see also figure 13). It increases, though, with an increasing level of excitation and consequent spatial wave function distribution. It follows that the low-energetic PL is fairly insensitive to the PEP. Figure 23 also shows that the holes confined to the exterior PEP minima (see figure 12) do not contribute to the QD PL.

Figure 24 shows the energies of the lowest QD PL peaks (solid line) of the SIQD shown in figure 1, as a function of the $\operatorname{In}_{x} \mathrm{Ga}_{1-x} \mathrm{As} \mathrm{QW}$ composition. Figure 24 corresponds to simulations with a fixed InP island size $\left(90.7 \times 56.7 \times 24 \mathrm{~nm}^{3}\right)$. The ground-state peak energy is to a large extent determined by the magnitude of the biaxial compressive strain in the $\mathrm{QW}$, in addition to the QW band gap energy. The peak-peak energy separation is, however, determined by the size of the stressor island alone. It follows that the material composition of the QW shifts the QD peak energies roughly equally. 

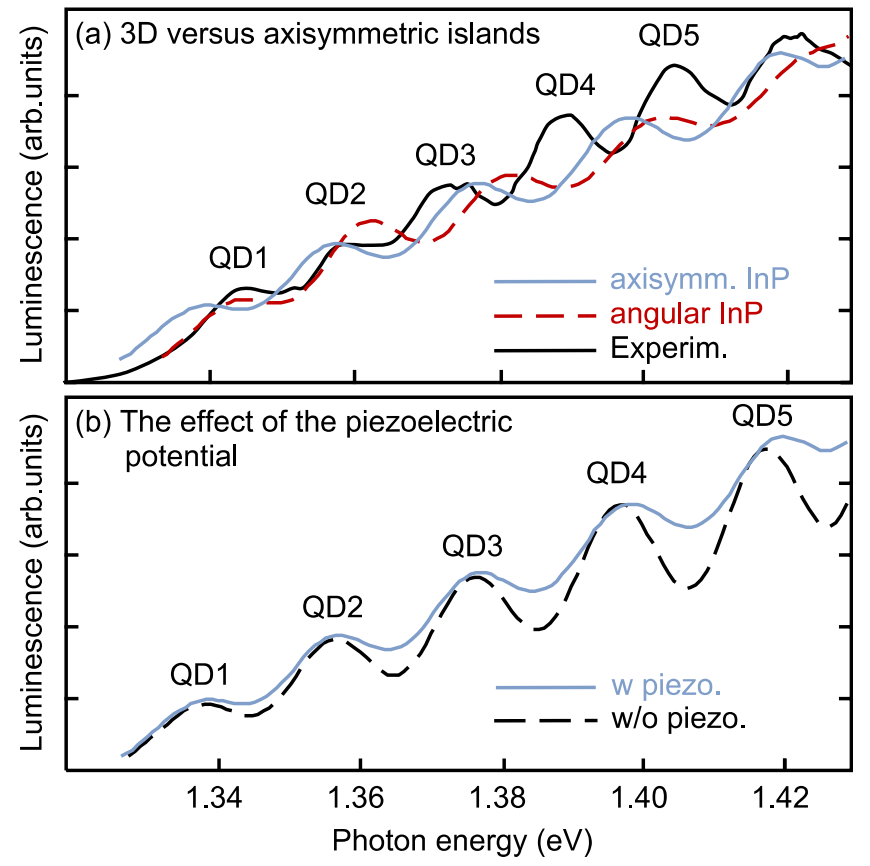

Figure 23. Simulated and experimental [4] CW PL data (black solid line). The blue (grey) solid line was computed with InP islands in the shape of truncated cones, whereas the red (grey) dashed line corresponds to simulations based on the InP island geometry of figure 1. (b) Photoluminescence of SIQD with axisymmetric InP island, accounting for the PEP (solid line) and neglecting the PEP (dashed line).

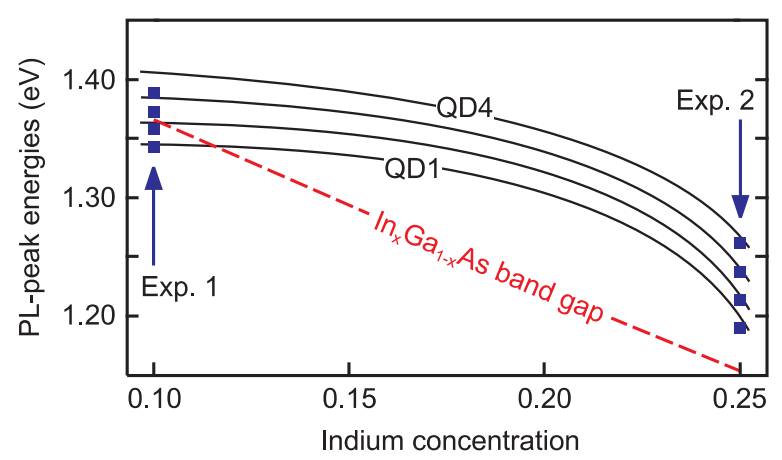

Figure 24. The energies of the lowest SIQD PL peaks (- ) as a function of the In concentration of the $\operatorname{In}_{x} \mathrm{Ga}_{1-x}$ As QW. The band gap energy of strain-free $\operatorname{In}_{x} \mathrm{Ga}_{1-x}$ As (- - - ) bulk material is shown for reference. The experimental data ( $\square)$ correspond to 1: [22] and 2: [4].

\subsection{Polarization}

Figure 25 shows the $x / y$ polarization of the QD PL, where $x \uparrow \uparrow\left[\begin{array}{lll}1 & 1 & 0\end{array}\right]$ and $y \uparrow \uparrow\left[\begin{array}{lll}1 & 10\end{array}\right]$ lie in the plane of the QW. The PL of SIQD shows only a very weak degree of $x / y$ polarization anisotropy as a result of the almost parabolic confinement potential. However, the PL has only a very small $z$ component. This is a result of the strong $\mathrm{QW}$ confinement, which increases the $\mathrm{HH}$ character of the hole states and causes $\left\langle\Psi_{\mathrm{e} i}\left|\hat{p}_{z}\right| \Psi_{\mathrm{h} f}\right\rangle$ to be small. 


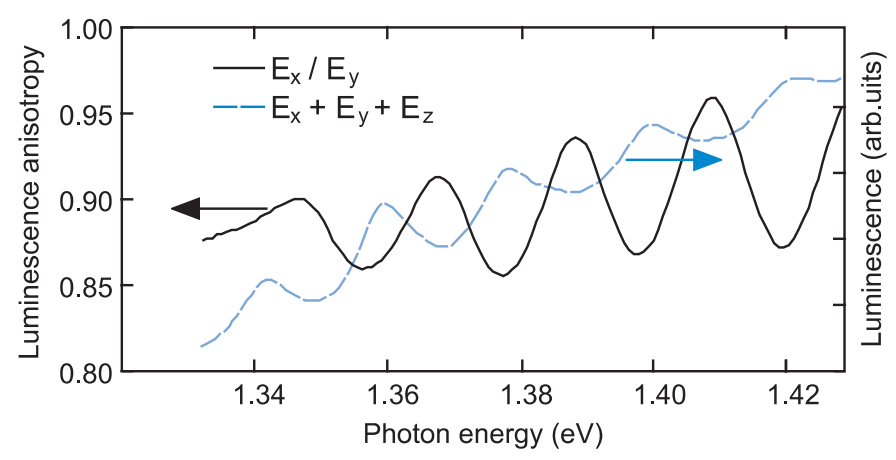

Figure 25. The $x / y$ polarization anisotropy (_- ) of the PL as a function of the photon energy. The total PL (averaged over the polarization) is given for reference (- - - -).

\section{Carrier dynamics}

Figure 26 depicts schematically the carrier dynamics in a SIQD and the surrounding QW under $\mathrm{CW}$ excitation. Note that the energy levels of figure 26 are schematic; a more realistic picture of the electron (hole) DOS was given in figure 12. In a typical PL experiment, the carriers are created by an $\mathrm{Ar}^{+}$laser with a photon energy well above the QW band gap. This mainly generates carriers in the bulk barrier material. The carriers are therefore initially 'hot'. They relax to the bulk band-edge and subsequently diffuse to the SIQD either directly or through the QW state continuum. The radiative recombination lifetime of QW carriers is of the order of $\tau \sim 0.5 \mathrm{~ns}$. Within the QW, some of the carriers get trapped by the SIQD. The capture of carriers from the QW to the QD proceeds mainly through Coulomb scattering and LO phonon emission. The efficiency of the Coulomb scattering depends on the carrier density of the 2DEG. The lifetime of the LO-phonon assisted QD capturing is of the order of $\tau \sim 1 \mathrm{ps}$.

Within an SIQD, the electrons (holes) can either relax towards the QD ground-state, which is about $95 \mathrm{meV}(10 \mathrm{meV})$ below the QW band-edge, or recombine radiatively with a typical radiative lifetime of $\tau \sim 1 \mathrm{~ns}$. The phonon-assisted relaxation of electrons is very inefficient as a result of the $\sim 14 \mathrm{meV}$ energy level spacing, whereas the dense hole DOS (energy level spacing of $\sim 2 \mathrm{meV}$ ) supports LA phonon-assisted hole relaxation. It follows that the intraband relaxation of confined electrons and holes proceeds through, e.g. Auger processes, where an electron relaxes by exciting a hole and subsequent phonon-assisted hole relaxation.

\subsection{Phonon relaxation and Auger processes in $S I Q D$}

The requirement for simultaneous energy and momentum conservation in the phonon scattering of carriers confined to QD has been predicted by Bockelmann and Bastard [131] and by Benisty et al [132] as being likely to slow down the intra-band carrier relaxation. This phenomenon is called the phonon bottleneck and follows from a reduced accessible phase space of scattered charge carriers. Even though the analyses of [131,132] were based on an infinite square-well confinement potential the conclusions are likely to hold true for SIQD as well.

The LO phonon relaxation is slow since the LO phonon DOS is narrow and outside it the transition rate fades out rapidly [131]. Accordingly, if the conduction band level spacing does not fit with the LO phonon DOS this relaxation mechanism is significantly reduced. For LA relaxation it has been shown that the scattering amplitude is reduced by simultaneous conservation of energy and momentum. Braskén et al have calculated the electron LA 


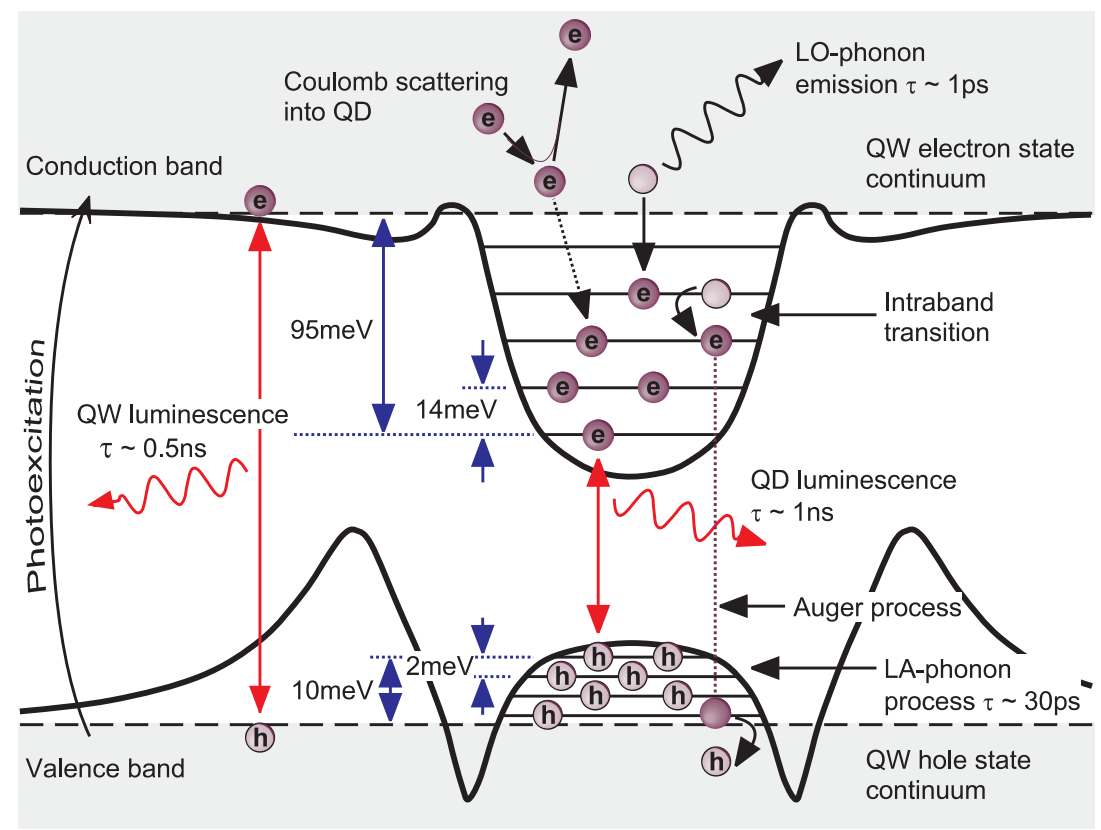

Figure 26. Intra- and inter-band carrier processes in strain-induced quantum dots. The energy scale and the line-up of the electron (hole) levels is only schematic.

relaxation time to be of the order of $\tau_{\mathrm{LO}} \approx 10^{-6} \mathrm{~s}$ at low state filling and zero temperature [133]. A conclusive verification of the phonon bottleneck is difficult since the Auger processes become very fast even with a modest state filling, thus obscuring the reduction of the phonon relaxation rate [28]. It is also noteworthy that some experiments have shown relaxation rates that are much faster [27], whereas others have shown rates much slower [134] than the calculated LA phonon emission rates [133].

The holes are efficiently relaxed by LA phonon emission, since the energy spacing of the hole states is much smaller. Auger processes, involving the excitation of a hole from a QD state to the QW continuum and the simultaneous de-excitation of an electron from its excited QD states to the QD electron ground-state, therefore, becomes an efficient carrier relaxation mechanism [28]. Figure 27 shows a comparison of the simulated and experimentally observed decline of luminescence lines after a short intensive laser excitation pulse [26]. The theoretical predictions correspond to simulations with a constant intra-band relaxation time $\tau_{0}=570 \mathrm{ps}$ and a time constant of $\tau_{q}=1$ ps for intra-band relaxation mediated by Coulomb scattering between a carrier in the QD and in the QW. Experimental determination of the intra-band phonon relaxation time has been reported only recently [135] and confirms the theoretical predictions above. Further understanding of phonon dynamics calls for truly atomistic phonon mode calculations [136] and perhaps for a new atomistic theory of electron phonon scattering.

\subsection{Dynamical model describing carrier modulation by $\mathrm{THz}$ radiation}

The presence of the PEP minima makes the relaxation pathways very different from conventional QD carrier dynamics, especially for holes. The piezoelectric side barriers (shown in figures 9 and 10) block the relaxation of holes to the DP minimum along the $y$-axis. The 


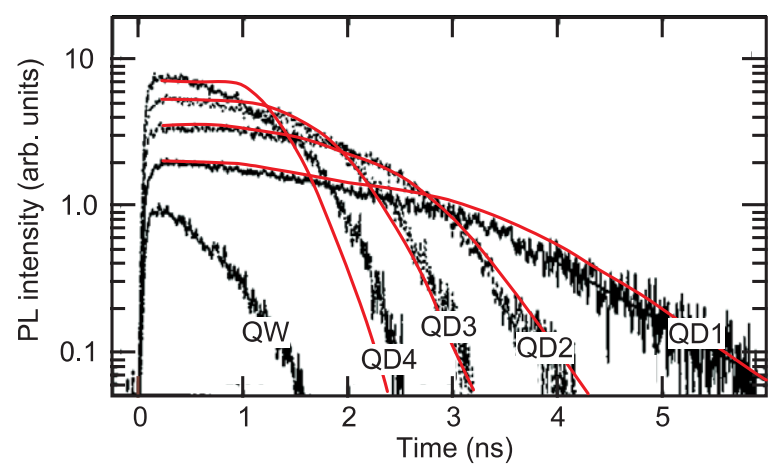

Figure 27. Time-resolved PL spectra of the four lowest QD transitions and of the QW for a high excitation condition. The ground-state transition is indicated by QD1, the first excited state by QD2, etc. The smooth lines correspond to simulations with a constant intra-band relaxation time and which account for Coulomb scattering between carriers of the QD and the QW. (Reprinted with permission from [27].)

relaxation along the $x$-axis is in turn hindered by the PEP minima which capture the holes before they can enter the DP minima.

Yusa et al studied the intra-band dynamics of SIQD in a spectroscopic experiment that made use of simultaneous optical carrier generation with $\mathrm{Ar}^{+}$(or Ti-Sap) laser and $\mathrm{THz}$ radiation carrier modulation using a FEL (the FEL energy of the experiments was $\hbar \omega_{\mathrm{THz}}=10.4 \mathrm{meV}$ ) [137]. This experiment was recently analysed in terms of master equation simulations in [63]. The model of [63] is depicted schematically in figure 28. It is a generalization of the models presented in [138] and [27], which are not as such able to describe the experiments analysed here. The experiments of Yusa et al [137] showed that (i) the $\mathrm{THz}$ radiation increases the ground-state luminescence and reduces the excited state luminescence at low generation intensities during CW THz excitation. Simulations [63] showed that this is a result of a THz radiation-induced charge transfer from the PEP minima to the ground DP hole state; (ii) THz radiation was experimentally found to give rise to a flash of ground-state luminescence, long after the carrier generation had been turned off. According to the theory [63], this is due to a spatial charge separation of electrons and holes, which is released by the $\mathrm{THz}$ excitation of holes. A brief review of the model and the main results of [63] will be given below.

6.2.1. Underlying assumptions. Reference [63] was based on the following assumptions, which originate both from experiments and theoretical studies.

(i) The SIQD are charge-neutral, which implies that the total number of holes equals the total number of electrons. This is motivated for undoped samples under purely optical carrier generation and is supported by experiments $[4,118]$. Small charge fluctuations between different QD mainly broaden the PL peaks.

(ii) Non-radiative recombination can be neglected. The self-organized samples of SIQD are of very high crystal quality, showing very long luminescence lifetimes of the order of $1 \mathrm{~ns}$ [27]. This indicates that non-radiative recombination channels are rare.

(iii) The confined electron and hole states can be described in terms of a few degenerate levels. The complete spectrum of confined states is too broad to be included as a whole in a numerical MC model. The DOS was therefore simplified using ten degenerate electron and hole QD levels, which effectively resembles the full 3D multi-band DOS. 


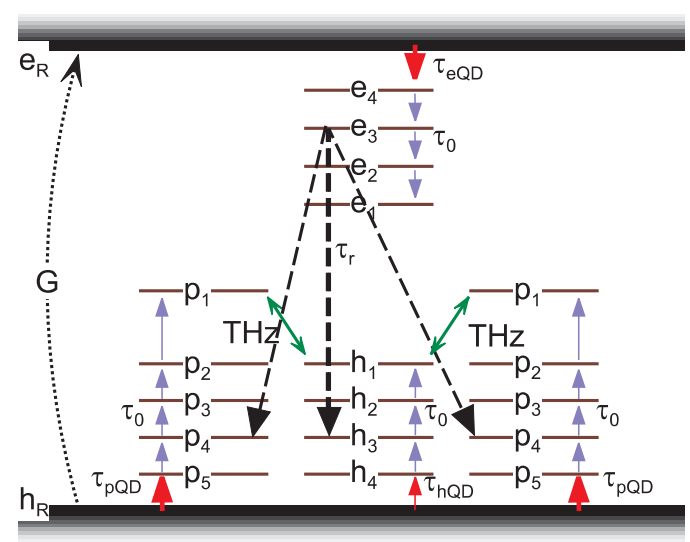

Figure 28. Carrier dynamics model of electrons and holes in SIQD [63]. The eigenstates of the DP minima are labelled $\left|e_{i}\right\rangle$ and $\left|h_{i}\right\rangle$; the hole states of the PEP minima are labelled $\left|p_{i}\right\rangle$. The pump laser $G(\ldots \ldots)$ generates carriers to the $\mathrm{QW}$ reservoirs $\left(\left|e_{\mathrm{R}}\right\rangle\right.$ and $\left.\left|h_{\mathrm{R}}\right\rangle\right)$ and the resonant $\mathrm{THz}$ radiation transfers holes between the ground-states $\left|p_{1}\right\rangle$ and $\left|h_{1}\right\rangle$ (double-headed arrows). Radiative recombinations (dashed arrows) are shown for the third electron level only. The energy scale is only schematic.

(iv) The spin is a good quantum number and is conserved in every intra-band relaxation and inter-band recombination process. This is motivated by the strain-induced HH-LH band splitting (see figure 3), which favours the confinement of $\mathrm{HH}$ with a well-defined spin. This effectively reduces partial relaxation and recombination rates.

(v) The intra-band relaxation rate $\left(1 / \tau_{0}\right)$ is ten times larger than the radiative recombination rate $\left(1 / \tau_{r}\right)$ at high state filling [27,28]. Equal intra-band relaxation times of both electrons and holes are also motivated for high carrier densities in which the carrier relaxation is dominated by Coulomb scattering and Auger-type relaxation [27, 28].

(vi) The carrier tunnelling between the PEP and DP minima can be neglected as this tunnelling rate is small in comparison with the prevailing relaxation and recombination rates. The spatial separation of the DP and PEP minima is about $20-30 \mathrm{~nm}$.

(vii) Intense THz radiation couples the hole populations of states $\left|p_{1}\right\rangle$ and $\left|h_{1}\right\rangle$ if the THz photon energy matches the energy separation of $\left|h_{1}\right\rangle$ and $\left|p_{1}\right\rangle$. It gives rise to a resonant charge transfer where the absorption (hole transition $\left|p_{1}\right\rangle \rightarrow\left|h_{1}\right\rangle$ ) and emission $\left(\left|h_{1}\right\rangle \rightarrow\left|p_{1}\right\rangle\right.$ ) of $\mathrm{THz}$ photons compete. The strength of the hole transfer is, in the experiment, not limited to a very narrow $\mathrm{THz}$ frequency range since in a real SIQD sample the single resonance frequency of the model is replaced by a quasi-continuum of transition frequencies as a result of the inhomogeneous linewidth broadening.

6.2.2. General remarks on the model parametrization. All the model parameters, except for the phenomenological coupling, are based on direct measurements, fitting to experimental data, or electron structure calculations [4,27,28]. The available experimental and numerical tools are, however, currently far from making possible quantitative experimental or theoretical determination of the $\mathrm{THz}$ radiation coupling constant.

The radiative lifetimes were calculated in the electric dipole approximation using eightband electron structure simulations and were in good agreement with the experiments. The radiative recombination rates of the $\left|p_{1}\right\rangle$ states were found to be negligible. The radiative recombination rates of less excited PEP states were noticeably smaller than those of the DP 

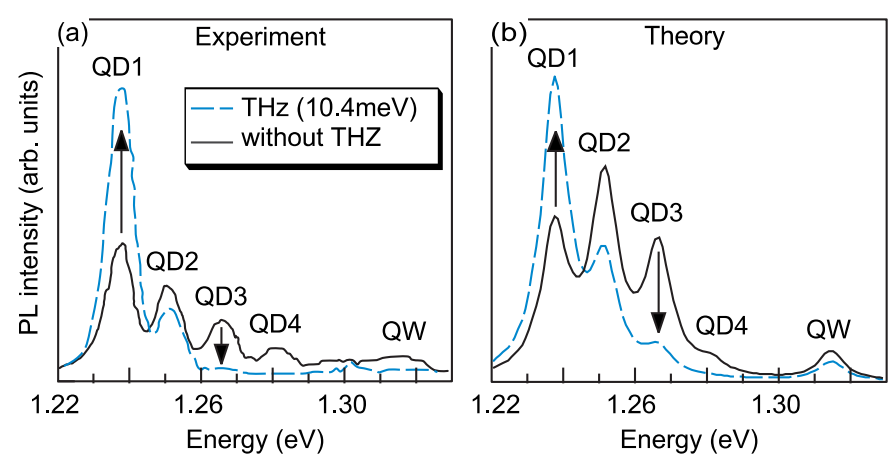

Figure 29. (a) Experimental [137] and (b) theoretical [63] QD PL during $\mathrm{Ar}^{+}$laser pumping (-) and during simultaneous $\mathrm{Ar}^{+}$laser pumping and FEL modulation (- - - ).

states. Effective recombination lifetimes of $\tau_{r i}^{\prime}=\tau_{r i} /\left(1-f_{i}\right)$ were used for the excited PEP states, where the values of $0<f_{i}<1$ were determined by fitting the state filling (PL peak intensities) to experimental data [118].

The validity of the radiative and intra-band relaxation time constants describing the carrier dynamics, in the absence of any $\mathrm{THz}$ radiation, was verified by comparing the numerical timedependent PL of our model with experiments. On the basis of this comparison we conclude that our model is very well in line with [118] and [27] (see also figure 23).

6.2.3. Luminescence and carrier dynamics during continuous pumping. The carriers generated to the GaAs barrier can relax to the DP and PEP minima, either directly or via QW states. The direct relaxation is assisted by the funnel-like deformation and piezoelectric potential energy minima, which extend into the barriers [13]. As the holes are predominantly confined to the deep PEP minima, the radiative QD recombination starts only when the PEP minima are already filled with holes. An SIQD is consequently strongly polarized during continuous generation and recombination. This results in a clear charge separation, with most holes confined to the PEP minima and all electrons confined to the DP minimum. This charge separation can persist even seconds after the carrier generation has been turned off.

6.2.4. Influence of $\mathrm{THz}$ radiation on steady-state $\mathrm{QD}$ luminescence. The enhancement of the ground-state (QD1) luminescence is due to a $\mathrm{THz}$ radiation-induced continuous drift of holes between the PEP $\left|p_{1}\right\rangle$ levels and the DP $\left|h_{1}\right\rangle$ level (figure 29) [63]. Under steady-state conditions the total integrated PL intensity is conserved in the first approximation. The QD2, QD3, QD4 and QW peaks are accordingly reduced by the $\mathrm{THz}$ radiation. The results are in good qualitative agreement with the experiments, although a quantitative agreement cannot be achieved with the current model. The large decrease in the QW PL in the experiments is related to the heating and ionization of QW excitons [139]. Our calculations predict, furthermore, that the $\mathrm{THz}$ radiation enhancement of the QD1 peak disappears at high $\mathrm{Ar}^{+}$pumping intensities as a result of the saturation of the hole population at $\left|h_{1}\right\rangle$.

6.2.5. Transient carrier dynamics under $\mathrm{THz}$ radiation. Figures $30(a)$ and $(b)$ show experimental and simulated time-resolved PL. The black and red (grey) curves correspond to the integrated PL of the QD1 and QW peaks, respectively. The QW peak is reduced, 

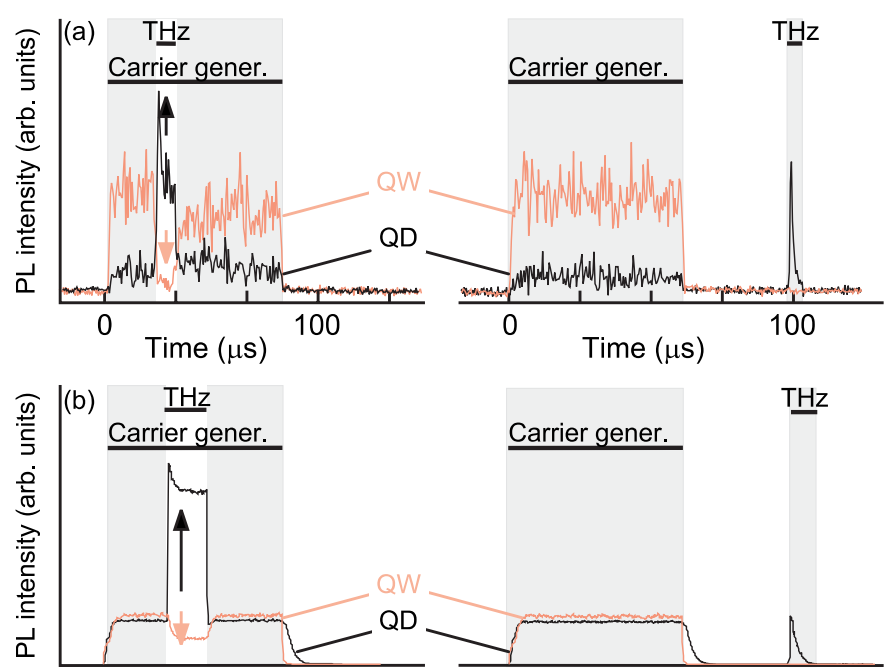

Figure 30. (a) Experimental [137] and (b) simulated [63] time-resolved PL of the QD ground-state (black) and QW (red/grey) luminescence at a temperature of $T=15 \mathrm{~K}$. The time windows of the Ti-Sap laser pumping and $\mathrm{THz}$ radiation are indicated by horizontal bars.

whereas the QD1 PL is increased during the THz radiation, with both PL peaks returning to their initial value after the $\mathrm{THz}$ radiation has been turned off. At the onset of the $\mathrm{THz}$ radiation, during $\mathrm{CW}$ carrier generation, there is a strong and sudden increase in the groundstate (QD1) luminescence (left-hand panel of figure 30). The enhancement by the $\mathrm{THz}$ radiation is then exponentially reduced, saturating at a lower level when the steady-state condition is reached between the recombination and relaxation processes. The energy and power of the $\mathrm{THz}$ radiation in the experiments were $\hbar \omega_{\mathrm{THz}}=2.5 \mathrm{meV}$ and $P=1 \mathrm{~kW}$, respectively [137].

6.2.6. $\mathrm{THz}$ radiation-induced delayed ground-state $P L$. The right-hand panels of figure 30 show PL with a delayed $\mathrm{THz}$ radiation pulse after the photo-excitation laser has been turned off. The rise in the delayed QD1 peak caused the THz radiation is due to the excitation and release of trapped holes from the PEP minima to the DP minimum, where they recombine with electrons localized in the DP minimum.

\section{Summary}

The electronic structure and carrier dynamics of SIQD, discovered in the early 1990s, are presently well understood at a phenomenological level. This holds true both for timeindependent and dynamic phenomena, as well as the single-particle and excitonic manyparticle regimes. However, the overall picture, although consistent, is still far from complete. Below we will summarize the main theoretical achievements and discuss the future outlook for SIQD.

The elastic strain induced by the InP stressor island was computed using axially symmetric and fully $3 \mathrm{D}$ elasticity models. The latter accounts for the geometry of the self-organized stressor islands. The latest elasticity models also include piezoelectric effects. Although no 
direct comparisons with atomistic models exist, for this particular semiconductor structure, we expect that the CE model is at least semi-quantitative.

The electronic structure was been modelled using the single-particle approximation and multi-band effective-mass model, accounting for the in-built elastic strain and piezoelectric potential, as well as external electro-magnetic fields. These calculations reproduce the experimental luminescence line energies, the Zeeman (small magnetic fields) and the diamagnetic (large magnetic fields) shifts of the SIQD spectrum. The QCSE was simulated for electric fields aligned in the plane of the QW, predicting a large anisotropic QD exciton polarizability.

The carrier correlation of the electron-hole states has been analysed with extensive manybody simulations using a two-band EMA model. The carrier-carrier correlation effects and addition energies of carriers in SIQD were computed using the CI for up to eight confined electron-hole pairs. Both neutral and charged carrier configurations were analysed. The many-body calculations are in surprisingly good agreement with mean-field calculations. The pair correlation function can still not be neglected, in spite of the small correlation energy.

Semi-empirical models were developed for the electron-hole dynamics of SIQD, reproducing all major QD PL experiments. The typical carrier dynamics models account both for the details of single QD and the statistical randomness of the carrier processes in an ensemble of many SIQD. These computations include both radiative recombination and intra-band carrier relaxation taking place through Auger-type relaxation, Coulomb scattering and hole relaxation via external PEP minima.

Despite the good overall progress we want to list open or only partially understood topics for further theoretical and experimental work. The experimentally observed clear and evenly distributed peaks in the QD PL are not due to the parabolic confinement potential alone. This is also a result of an inhomogeneously broadened superposition of many different recombination processes of various strengths. However, the peak-peak energy separation is predominantly determined by the energy separation of the electron states. This emphasizes the need for fully $3 \mathrm{D}$ electron structure calculations, also in studies of correlation effects and the modelling of the magnetoluminescence.

A large PEP is induced by the InP stressor island and gives rise to deep potential minima of holes. The PEP minima are, however, located outside the radiative electron and hole states and, as a consequence do not affect the steady-state QD PL, although they decisively influence the dynamics and intra-band relaxation of holes. A better understanding of the effects of the PEP definitely calls for further experiments. We predict that $\mathrm{CW}$ and time-resolved experiments devoted specifically to this issue could give a direct validation of the PEP and its role in carrier dynamics.

Most of the experimental observations so far have been analysed or explained by the theoretical and numerical models reviewed in this work. However, because of the PEP and large QD size, the physics of SIQD becomes more complex than in the case of smaller QD. We note that e.g. overgrown InAs pyramid QD or CdSe nanocrystal QD confine only a few electron-hole pairs during conventional PL measurements, whereas SIQD are much larger in size and can confine up to 20-30 electron-hole pairs. This makes it computationally more demanding to analyse SIQD using microscopic or first-principle models.

The interaction between confined carriers and different types of phonons should be studied using a first principle-type description of both the phonons and the carriers. Both the carrier dynamics and many-particle properties deserve further calculations, which should include the deep PEP minima. New theories and more advanced simulations should, however, be developed in parallel with the development of new experimental techniques as this would give a solid verification of future theoretical findings. 


\section{Acknowledgments}

We are grateful to Bradley Foreman of the Hong Kong University of Science and Technology for valuable communications and comments on electron structure calculations using the BurtForeman effective-mass approximation. We thank Dage Sundholm of the University of Helsinki for his help and comments regarding the many-particle simulations. We are also indebted to Harri Lipsanen and Markku Sopanen of Helsinki University of Technology for fruitful collaboration and inspiring discussions.

\section{References}

[1] Bimberg D, Grundmann M and Ledentsov N N 1999 Quantum Dot Heterostructures (New York: Wiley)

[2] Kouwenhoven L and Marcus C 1998 Quantum dots Phys. World 11 35-9

[3] Ashoori R C 1996 Electrons in artificial atoms Nature 379 413-19

[4] Lipsanen H, Sopanen M and Ahopelto J 1995 Luminescence from excited states in strain-induced $\operatorname{In}_{x} \mathrm{Ga}_{1-x} \mathrm{As}_{\mathrm{s}}$ quantum dots Phys. Rev. B 51 13868-71

[5] Adler F, Geiger M, Bauknecht A, Scholz F, Schweizer H, Pilkuhn M H, Ohnesorge B and Forchel A 1996 Optical transitions and carrier relaxation in self assembled InAs/GaAs quantum dots J. Appl. Phys. 80 4019-26

[6] Bayer M, Hawrylak P, Hinzer K, Fafard S, Korkusinski M, Wasilewski Z R, Stern O and Forchel A 2001 Coupling and entangling of quantum states in quantum dot molecules Science 291 451-3

[7] Badolato A, Hennessy K, Atatüre M, Dreiser J, Hu E, Petroff P M and Imamoglu A 2005 Deterministic coupling of single quantum dots to single nanocavity modes Science 308 1158-61

[8] Stier O 2001 Electronic and Optical Properties of Quantum Dots and Wires (Berlin: Wissenschaft und Technik)

[9] Seifert W, Carlsson N, Miller M, Pistol M-E, Samuelson L and Wallenberg L R 1996 In-situ growth of quantum dot structures by the Stranski-Krastanow growth mode Prog. Cryst. Growth Charact. Mater. 33 423-71

[10] Stangl J, Holy V and Bauer G 2004 Structural properties of self-organised semiconductor nanostructures Rev. Mod. Phys. 76725

[11] Georgsson K, Carlsson N, Samuelson L, Seifert W and Wallenberg L R 1995 Transmission electron microscopy investigation of the morphology of InP Stranski-Krastanow islands grown by metalorganic chemical vapor deposition Appl. Phys. Lett. 67 2981-2

[12] Ahopelto J, Sopanen M and Lipsanen H 1999 Tailoring of energy levels in strain-induced quantum dots Japan. J. Appl. Phys. 38 1081-4

[13] Tulkki J and Heinämäki A 1995 Confinement effect in a quantum well dot induced by an InP stressor Phys. Rev. B 52 8239-43

[14] Ren H-W, Nair S V, Lee J-S, Sugou S, Okuno T, Nishbayashi K and Masumoto Y 2000 Confinement effects in strain-induced InGaAs/GaAs quantum dots Physica E 7 403-7

[15] Sopanen M, Taskinen M, Lipsanen H and Ahopelto J 1996 Red luminescence from strain-induced GaInP quantum dots Appl. Phys. Lett. $693393-5$

[16] Hanna M C, Lu Z H, Cahill A F, Heben M J and Nozik A J 1997 MOCVD growth and optical characterization of strain-induced quantum dots with InP island stressors J. Cryst. Growth 174 605-10

[17] Wang T and Forchel A 1998 Growth and optical investigation of strain-induced AlGaAs/GaAs quantum dots using self-organised GaSb islands as a stressor Appl. Phys. Lett. 73 1847-9

[18] Riikonen J, Sormunen J, Mattila M, Sopanen M and Lipsanen H 2005 InGaAs/InP quantum dots induced by self-organised InAs stressor-islands Japan. J. Appl. Phys. 44 L518-20

[19] Riikonen J, Sormunen J, Sopanen M and Lipsanen H 2005 Highly tunable emission from strain-induced InGaAsP/InP quantum dots Japan. J. Appl. Phys. 44 L976-8

[20] Koskenvaara H, Riikonen J, Sormunen J, Sopanen M and Lipsanen H 2006 Carrier dynamics in strain-induced InGaAsP/InP quantum dots Physica E 32 179-82

[21] Kim E S, Usami N and Shiraki Y 1997 Anomalous luminescence peak shift of SiGe/Si quantum well induced by self-assembled Ge islands Appl. Phys. Lett. 70 295-7

[22] Rinaldi R, Giugno P V, Cingolani R, Lipsanen H, Sopanen M, Tulkki J and Ahopelto J 1996 Zeeman effect in parabolic quantum dots Phys. Rev. Lett. 77 342-5

[23] Lipsanen H, Sopanen M and Tulkki J 2005 Optics of Quantum Dots and Wires (Boston, MA: Artech House) pp 97-131

[24] Rinaldi R et al 2001 Correlation effects in strain-induced quantum dots Phys. Status Solidi b 224 361-6 
[25] Obermuller C, Deisenrieder A, Abstreiter G, Karrai K, Grosse S, Manus S, Feldmann J, Lipsanen H, Sopanen M and Ahopelto J 1999 Pauli-blocking imaging of single strain-induced semiconductor quantum dots Appl. Phys. Lett. 74 3200-2

[26] Lipsanen H, Sopanen M, Tulkki J, Ahopelto J, Braskén M and Lindberg M 1999 Growth and optical properties of strain-induced quantum dots Phys. Scr. T 79 20-6

[27] Grosse S, Sandmann J H H, von Plessen G, Feldmann J, Lipsanen H, Sopanen M, Tulkki J and Ahopelto J 1997 Carrier relaxation dynamics in quantum dots: scattering mechanisms and state-filling effects Phys. Rev. B 55 4473-6

[28] Braskén M, Lindberg M, Sopanen M, Lipsanen H and Tulkki J 1998 Temperature dependence of carrier relaxation in strain-induced quantum dots Phys. Rev. B 58 R15993-6

[29] Heitz R, Born H, Guffarth F, Stier O, Schliwa A, Hoffmann A and Bimberg D 2001 Existence of a phonon bottleneck for excitons in quantum dots Phys. Rev. B 64241305

[30] Malvern L 1969 Introduction to the Mechanics of a Continuous Medium (Englewood Cliffs, NJ: Prentice-Hall)

[31] Lai W, Rubin D and Krempl E 1996 Introduction to Continuum Mechanics (Amsterdam: Elsevier)

[32] Brandt O, Ploog K, Bierwolf R and Hohenstein M 1992 Breakdown of continuum elasticity theory in the limit of monatomic films Phys. Rev. Lett. 68 1339-42

[33] Stier O, Grundmann M and Bimberg D 1999 Electronic and optical properties of strained quantum dots modelled by 8-band $\boldsymbol{k} \cdot \boldsymbol{p}$ theory Phys. Rev. B $595688-701$

[34] Pryor C, Kim J, Wang L W, Williamson A J and Zunger A 1998 Comparison of two methods for describing the strain profiles in quantum dots J. Appl. Phys. 83 2548-54

[35] v Alfthan S, Boxberg F, Kaski K, Kuronen A, Tereshonkov R, Tulkki J and Sakaki H 2005 Electronic, optical and structural properties of quantum wire superlattices on vicinal (1111) GaAs substrates Phys. Rev. B 72045329

[36] Rössler U and Strauch D 2001 Numerical Data and Functional Relationships in Science and Technology (Landolt-Börnstein. New Series III/17a) ed O Madelung, U Rössler and M Schulz (Berlin: Springer)

[37] Singh J 1993 Physics of Semiconductors and Their Heterostructures (New York: Mcgraw-Hill)

[38] Nye J F 1985 Physical Properties of Crystals (New York: Oxford University Press)

[39] Keating P N 1966 Effect of invariance requirements on the elastic strain energy of crystals with application to the diamond structure Phys. Rev. 145 637-45

[40] Martin R 1970 Elastic properties of ZnS structure semiconductors Phys. Rev. B 14005

[41] Nordlund K, Partyka P, Averback R S, Robinson I K and Ehrhart P 2000 Atomistic simulation of diffuse x-ray scattering from defects in solids J. Appl. Phys. 88 2278-88

[42] Pryor C, Kim J, Wang L W, Williamson A J and Zunger A 1998 Comparison of two methods for describing the strain profiles in quantum dots J. Appl. Phys. 83 2548-54

[43] Corni S, Braskén M, Lindberg M, Olsen J and Sundholm D 2003 Electron-hole recombination density matrices obtained from large configuration-interaction expansions Phys. Rev. B 67085314

[44] Virkkala R, Maijala K and Tulkki J 2000 Piezoelectric potentials and carrier lifetimes in strain-induced quantum well dots Phys. Rev. B 62 6932-5

[45] Boxberg F, Tereshonkov R and Tulkki J 2006 Polarisation of gain and symmetry breaking by interband coupling in quantum well lasers J. Appl. Phys. 100063108

[46] Bastard G 1992 Wave Mechanics Applied to Semiconductor Heterostructures (New York: Wiley)

[47] Bir G L and Pikus G E 1974 Symmetry and Strain-Induced Effects in Semiconductors (New York: Wiley)

[48] Kane E O 1982 Energy band theory Handbook on Semiconductors vol 1 (Amsterdam: North-Holland) pp 193217

[49] Gershoni D, Henry C H and Baraff G A 1993 Calculating the optical properties of multidimensional heterostructures: application to the modeling of quaternary quantum well lasers IEEE J. Quantum Electron. 29 2433-49

[50] Bahder T B 1990 Eight-band $\boldsymbol{k} \cdot \boldsymbol{p}$ model of strained zinc-blende crystals Phys. Rev. B 41 11992-2001

[51] Bahder T B 1991 Analytic dispersion relations near the Gamma point in strained zinc-blende crystals Phys. Rev. B 45 1629-37

[52] Luttinger J M and Kohn W 1955 Motion of electrons and holes in perturbed periodic fields Phys. Rev. 97 869-83

[53] Löwdin P 1951 A note on the quantum-mechanical perturbation theory J. Chem. Phys. 19 1396-401

[54] Loehr J P 1995 Parameter consistency in multienergetic $\boldsymbol{k} \cdot \boldsymbol{p}$ models Phys. Rev. B 52 2374-80

[55] Enders P, Bärwolff A, Woerner M and Suisky D $1995 \boldsymbol{k} \cdot \boldsymbol{p}$ theory of energy bands, wave functions and optical selection rules in strained tetrahedral semiconductors Phys. Rev. B 51 16695-704

[56] Smith D L and Mailhiot C 1990 Theory of semiconductor superlattice electronic structure Rev. Mod. Phys. 62 173-234 
[57] Stier O and Bimberg D 1997 Modeling of strained quantum wires using eight-band $\boldsymbol{k} \cdot \boldsymbol{p}$ theory Phys. Rev. B $557726-32$

[58] Grundmann M, Stier O, Schliwa A and Bimberg D 2000 Electronic structure of cleaved-edge-overgrowth strain-induced quantum wires Phys. Rev. B 61 1744-7

[59] Pryor C 1998 Eight-band calculations of strained InAs/GaAs quantum dots compared with one-, four- and six-band approximations Phys. Rev. B 57 7190-5

[60] Banin U, Lee C J, Guzelian A A, Kadavanich A V, Alivisatos A P, Jaskolski W, Bryant G W, Efros A L and Rosen M 1998 Size-dependent electronic level structure of InAs nanocrystal quantum dots: test of multiband effective mass theory J. Chem. Phys. 109 2306-9

[61] Heitz R, Stier O, Mukhametzhanov I, Madhukar A and Bimberg D 2000 Quantum size effect in self-organised InAs/GaAs quantum dots Phys. Rev. B 62 11017-28

[62] Holm M, Pistol M-E and Pryor C 2002 Calculations of the electronic structure of strained InAs quantum dots in InP J. Appl. Phys. 92 932-6

[63] Boxberg F, Tulkki J, Yusa G and Sakaki H 2007 Cooling of radiative quantum-dot excitons by terahertz radiation: a spin-resolved Monte Carlo carrier dynamics model Phys. Rev. B 75115334

[64] Jiang H and Singh J 1998 Self-assembled semiconductor structures: Electronic and optoelectronic properties IEEE J. Quantum Electron. 34 1188-96

[65] Kane E 1957 Band structure of indium antimonide J. Phys. Chem. Solids 1 249-61

[66] Ando T and Akera H 1989 Connection of envelope functions at semiconductor heterointerfaces: II. Mixings of $\Gamma$ and $X$ valleys in GaAs/ $\mathrm{Al}_{x} \mathrm{Ga}_{1-x}$ As Phys. Rev. B 40 11619-33

[67] Laikhtman B 1992 Boundary conditions for envelope functions in heterostructures Phys. Rev. B 46 4769-74

[68] Einevoll G T and Sham L J 1994 Boundary conditions for envelope functions at interfaces between dissimilar materials Phys. Rev. B 49 10533-43

[69] Godfrey M J and Malik A M 1996 Boundary conditions and spurious solutions in envelope-function theory Phys. Rev. B 53 16504-9

[70] Rodina A V, Alekseev A Y, Efros A L, Rosen M and Meyer B K 2002 General boundary conditions for the envelope function in the multiband $\boldsymbol{k} \cdot \boldsymbol{p}$ model Phys. Rev. B 65

[71] Tokatly I V, Tsibizov A G and Gorbatsevich A A 2002 Interface electronic states and boundary conditions for envelope functions Phys. Rev. B 65165328

[72] Pistol M-E 2002 Boundary conditions in the envelope function approximation as applied to semiconductor heterostructures: the multi-band case J. Phys. Soc. Japan 71 1325-31

[73] Burt M G 1992 The justification for applying the effective-mass approximation to microstructures $J$. Phys.: Condens. Matter 4 6651-90

[74] Burt M G 1999 Fundamentals of envelope function theory for electronic states and photonic modes in nanostructures J. Phys.: Condens. Matter 11 53-83

[75] Foreman B A 1993 Effective-mass Hamiltonian and boundary conditions for the valence bands of semiconductor microstructures Phys. Rev. B 48 4964-7

[76] Foreman B A 1997 Elimination of spurious solutions from eight-band $\boldsymbol{k} \cdot \boldsymbol{p}$ theory Phys. Rev. B 56 R12748-51

[77] Foreman B A 2007 private communications

[78] Foreman B A 2005 First-principles envelope-function theory for lattice-matched semiconductor heterostructures Phys. Rev. B 72165345

[79] Dresselhaus G, Kip A F and Kittel C 1995 Cyclotron resonance of electrons and holes in silicon and germanium crystals Phys. Rev. 98 368-84

[80] Pidgeon C R and Brown R N 1966 Interband magneto-absorption and Faraday rotation in InSb Phys. Rev. $146575-83$

[81] Mlinar V, Tadic M, Partoens B and Peeters F M 2005 Nonsymmetrised Hamiltonian for semiconducting nanostructures in a magnetic field Phys. Rev. B 71205305

[82] Meney A T, Gonul B and OŔeilly E P 1994 Evaluation of various approximations used in the envelope-function method Phys. Rev. B 50 10893-904

[83] Boujdaria K, Ridene S, Radhia S B, Bouchriha H and Fishman G 2004 Symmetrised Hamiltonian versus Foreman Hamiltonian for semiconductor valence band: an insight Solid State Commun. 129 221-6

[84] Lassen B, Voon L L Y, Willatzen M and Melnik R 2004 Exact envelope-function theory versus symmetrised Hamiltonian for quantum wires: a comparison Solid State Commun. 132 141-9

[85] Franceschi S D, Jancu J-M and Beltram F 1999 Boundary conditions in multiband $\boldsymbol{k} \cdot \boldsymbol{p}$ models: a tight-binding test Phys. Rev. B 59 9691-4

[86] Kolokolov K I, Li J and Ning C Z $2003 k \cdot p$ Hamiltonian without spurious-state solutions Phys. Rev. B 68161308 
[87] Landau L D and Lifshitz E M 1999 Quantum Mechanics (Ser. Course of Theoretical Physics) (Burlington, MA: Butterworth-Heinemann) vol 3

[88] Braskén M, Lindberg M and Tulkki J 1997 Magnetoluminescence from strain-induced quantum dots Phys. Rev. B 55 9275-8

[89] Grundler D 2000 Large Rashba splitting in InAs quantum wells due to electron wave function penetration into the barrier layers Phys. Rev. Lett. 84 6074-7

[90] Bychkov Y A and Rashba E I 1984 Oscillatory effects and the magnetic susceptibility of carriers in inversion layers J. Phys. C: Solid State Phys. 17 6039-45

[91] Rinaldi R, Giorgi M D, Vittorio M D, Melcarne A, Visconti P, Cingolani R, Lipsanen H, Sopanen M, Drufva T and Tulkki J 2001 Longitudinal Stark effect in parabolic quantum dots Japan. J. Appl. Phys. 40 2002-5

[92] Empedocles S A and Bawendi M G 1997 Quantum-confined Stark effect in single CdSe nanocrystallite quantum dots Science 278 2114-17

[93] Seufert J, Obert M, Scheibner M, Gippius N A, Bacher G, Forchel A, Passow T, Leonardi K and Hommel D 2001 Stark effect and polarizability in a single CdSe/ZnSe quantum dot Appl. Phys. Lett. 79 1033-5

[94] Fu H 2002 Stark shifts, band-edge transitions and intrinsic optical dipoles in spherical InP quantum dots under electric fields Phys. Rev. B $\mathbf{6 5} 045320$

[95] Wolst O, Schardt M, Kahl M, Malzer S and Dhler G H 2002 A combined investigation of lateral and vertical Stark effect in InAs self-assembled quantum dots in waveguide structures Physica E 13 283-8

[96] Htoon H, Keto J W, Baklenov O, Holmes J A L and Shih C K 2000 Cross-sectional nanophotoluminescence studies of Stark effects in self-assembled quantum dots Appl. Phys. Lett. 76 700-2

[97] Heller W, Bockelmann U and Abstreiter G 1998 Electric-field effects on excitons in quantum dots Phys. Rev. B 57 6270-3

[98] Rinaldi R, Antonaci S, DeVittorio M, Cingolani R, Hohenester U, Molinari E, Lipsanen H and Tulkki J 2000 Effects of few-particle interaction on the atomic like levels of a single strain-induced quantum dot Phys. Rev. B 62 1592-5

[99] Rinaldi R, Mangino R, Cingolani R, Lipsanen H, Sopanen M, Tulkki J, Braskeń M and Ahopelto J 1998 Magneto-optical properties of strain-induced $\operatorname{In}_{x} \mathrm{Ga}_{1-x}$ As parabolic quantum dots Phys. Rev. B 57 9763-9

[100] Cingolani R et al 1999 Electron-hole correlation in quantum dots under a high magnetic field (up to 45 T) $P$ hys. Rev. Lett. 83 4832-5

[101] Bockelmann U 1994 Phonon scattering between zero-dimensional electronic states: spatial versus Landau quantization Phys. Rev. B 50 17271-9

[102] Cingolani R et al 2000 Influence of Coulomb and exchange interaction on quantum dot magnetoluminescence up to $B=45$ T Phys. Status Solidi a $178263-8$

[103] Cingolani R et al 2000 Effects of electron-hole correlation in quantum dots under high magnetic field (up to 45 T) Physica E 7 346-9

[104] Braskén M, Lindberg M, Sundholm D and Olsen J 2001 Spatial carrier-carrier correlations in strain-induced quantum dots Phys. Rev. B 64035312

[105] Braskén M, Corni S, Lindberg M, Olsen J and Sundholm D 2002 Full configuration interaction studies of phonon and photon transition rates in semiconductor quantum dots Mol. Phys. 100 911-18

[106] Braskén M, Lindberg M, Sundholm D and Olsen J 2001 Full configuration interaction calculations of electronhole correlation effects in strain-induced quantum dots Phys. Status Solidi b 224 775-9

[107] Braskén M 1999 Modeling of the optical properties of strain-induced quantum dots PhD Dissertation Åbo Akademi, Åbo, Finland

[108] Vänskä T, Lindberg M, Olsen J and Sundholm D 2006 Computational methods for studies of multiexciton complexes Phys. Status Solidi b 243 4035-45

[109] Barenco A and Dupertuis M A 1995 Quantum many-body states of excitons in a small quantum dot Phys. Rev. B 52 2766-78

[110] Dekel E, Gershoni D, Ehrenfreund E, Garcia J M and Petroff P M 2000 Carrier-carrier correlations in an optically excited single semiconductor quantum dot Phys. Rev. B 61 11009-20

[111] Shumway J, Franceschetti A and Zunger A 2001 Correlation versus mean-field contributions to excitons, multiexcitons and charging energies in semiconductor quantum dots Phys. Rev. B 63155316

[112] Narvaez G A, Bester G and Zunger A 2005 Excitons, biexcitons and trions in self-assembled (In,Ga)As/GaAs quantum dots: Recombination energies, polarisation and radiative lifetimes versus dot height Phys. Rev. B 72245318

[113] Sheng W, Cheng S-J and Hawrylak P 2005 Multiband theory of multi-exciton complexes in self-assembled quantum dots Phys. Rev. B 71035316

[114] Braskén M, Lindberg M, Sundholm D and Olsen J 2001 Atoms, Molecules and Quantum Dots in Laser Fields: Fundamental Processes vol 71, ed N Bloembergen et al (Bologna: Societ Italiana di Fisica) p 315 
[115] McWeeny R 1992 Methods of Molecular Quantum Mechanics (New York: Academic)

[116] Zunger A 2001 Pseudopotential theory of semiconductor quantum dots Phys. Status Solidi b 224 727-34

[117] Haken H 1976 Quantum Field Theory of Solids. An Introduction (Amsterdam: North-Holland)

[118] Lipsanen H, Sopanen M and Ahopelto J 1996 Fabrication and photoluminescence of quantum dots induced by strain of self-organised stressors Solid-State Electron. 40 601-4

[119] Corni S, Braskén M, Lindberg M, Olsen J and Sundholm D 2003 Stabilization energies of charged multiexciton complexes calculated at configuration interaction level Physica E 18 436-42

[120] He L and Zunger A 2006 Multiple charging of InAs/GaAs quantum dots by electrons or holes: addition energies and ground-state configurations Phys. Rev. B 73115324

[121] Szabo A and Saika A 1989 Modern Quantum Chemistry (New York: Dover)

[122] Jensen F 1999 Introduction to Computational Chemistry New York: Wiley)

[123] Braskén M, Lindberg M, Sundholm D and Olsen J 2000 Full configuration interaction calculations of electronhole correlation effects in strain-induced quantum dots Phys. Rev. B 61 7652-5

[124] Lüscher S, Heinzel T, Ensslin K, Wegscheider W and Bichler M 2001 Signatures of spin pairing in chaotic quantum dots Phys. Rev. Lett. $862118-21$

[125] Braskén M, Lindberg M, Sundholm D and Olsen J 2000 Carrier-carrier correlations in strain-induced quantum dots Phys. Status Solidi b 221 37-41

[126] Maksym P A, Imamura H, Mallon G P and Aoki H 2000 Molecular aspects of electron correlation in quantum dots J. Phys.: Condens. Matter 12 R299-334

[127] Reaves C M, Bressler-Hill V, Varma S, Weinberg W H and DenBaars S P 1995 Characterization of MOCVDgrown InP on InGaP/GaAs(001) Surf. Sci. 326 209-17

[128] Reaves C M, Pelzel R I, Hsueh G C, Weinberg W H and DenBaars S P 1996 Formation of self-assembled InP islands on a GaInP/GaAs(311)A surface Appl. Phys. Lett. $693878-80$

[129] Grundmann M and Bimberg D 1997 Gain and threshold of quantum dot lasers: theory and comparison to experiment Japan. J. Appl. Phys. 36 4181-7

[130] Qasaimeh O 2003 Effect of inhomogeneous line broadening on gain and differential gain of quantum dot lasers IEEE Trans. Electron Devices 50 1575-81

[131] Bockelmann U and Bastard G 1990 Phonon scattering and energy relaxation in two-, one- and zero-dimensional electron gases Phys. Rev. B $428947-51$

[132] Benisty H, Sotomayor-Torrès C M and Weisbuch C 1991 Intrinsic mechanism for the poor luminescence propeties of quantum-box systems Phys. Rev. B 44 10945-8

[133] Braskén M, Lindberg M and Tulkki J 1997 Carrier dynamics in strain-induced quantum dots Phys. Status Solidi a 164 427-30

[134] Gfroerer T H, Sturge M D, Kash K, Yater J A, Plaut A S, Lin P S D, Florez L T, Harbison J P, Das S R and Lebrun L 1996 Slow relaxation of excited states in strain-induced quantum dots Phys. Rev. B 53 16474-80

[135] Urayama J, Norris T B, Singh J and Bhattacharya P 2001 Observation of phonon bottleneck in quantum dot electronic relaxation Phys. Rev. Lett. 86 4930-3

[136] Fu H, Ozolinš V and Zunger A 1999 Phonons in GaP quantum dots Phys. Rev. B 59 2881-7

[137] Yusa G, Allen S J, Davies J, Sakaki H, Kono H, Ahopelto J, Lipsanen H, Sopanen M and Tulkki J 1998 Teraherznear infrared upconversion in strain-induced quantum dots 24th Int. Conf. on the Physics of Semiconductors (ICPS24), Jerusalem, Israel p 1083

[138] Grundmann M and Bimberg D 1997 Theory of random population for quantum dots Phys. Rev. B 55 9740-5

[139] Cerne J, Kono J, Sherwin M S, Sundaram M, Gossard A C and Bauer G E W 1996 Terahertz dynamics of excitons in GaAs/AlGaAs quantum wells Phys. Rev. Lett. 77 1131-4 